\title{
Towards Individualised Treatment of Tuberculosis
}

\section{Katarina Niward}

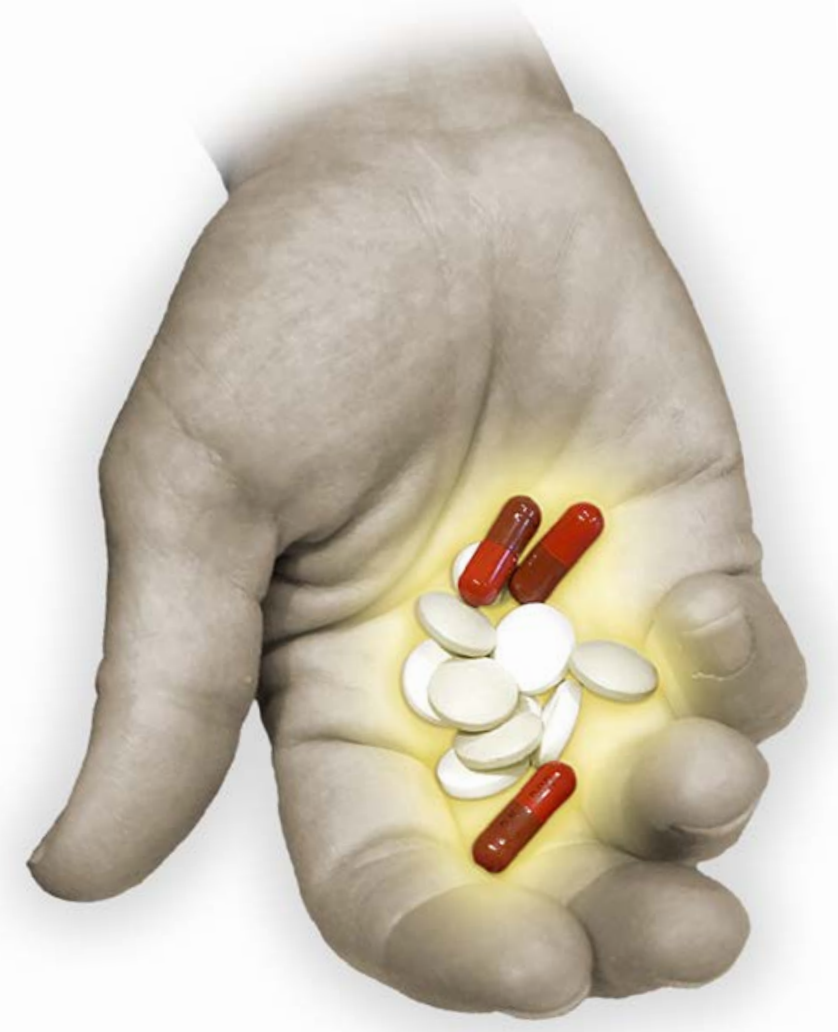




\title{
TOWARDS INDIVIDUALISED TREATMENT OF TUBERCULOSIS
}

\author{
Katarina Niward
}

\author{
Department of Infectious Diseases \\ Department of Clinical and Experimental Medicine \\ Linköping University, Sweden
}

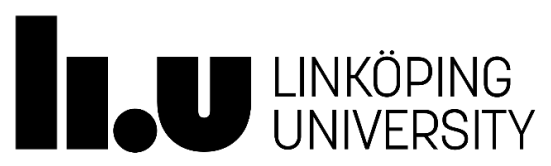

Linköping 2019 
(C) Katarina Niward, 2019

Front cover-design Katarina Niward and Tomas Hägg, LiU-Tryck, Linköping Published papers have been reprinted with the permission from the copyright holder. All illustrations unless otherwise specified are made by the author.

Printed in Sweden by LiU-Tryck, Linköping 2019.

ISBN 978-91-7685-128-9

ISSN 0345-0082 
To all who have suffered and lost their health, a loved one or their own life due to TB. 



\section{TABLE OF CONTENTS}

ABSTRACT

POPULÄRVETENSKAPLIG SAMMANFATTNING

LIST OF SCIENTIFIC PAPERS

ABBREVIATIONS

PERSONAL PREFACE

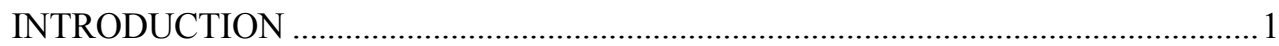

Tuberculosis from a global perspective .................................................................... 1

Tuberculosis - basic concepts .................................................................................. 3

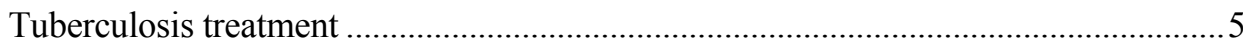

Drug susceptibility testing of $M$. tuberculosis ........................................................... 14

Treatment outcome and surrogate markers of clinical improvement ......................... 23

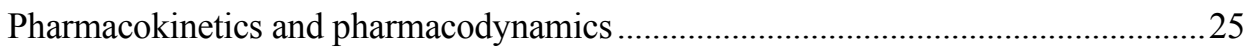

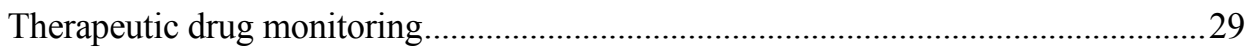

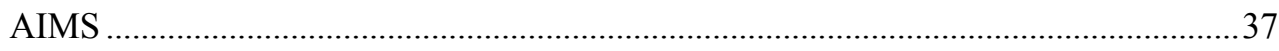

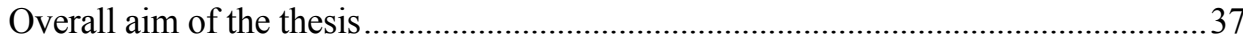

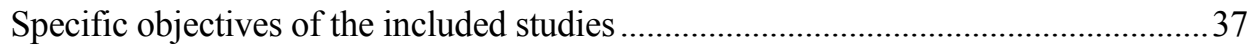

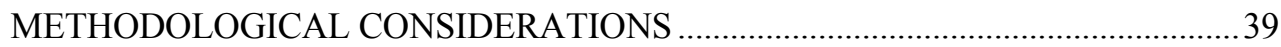

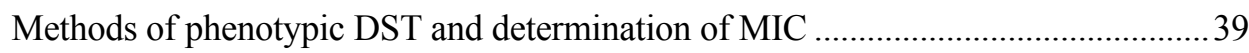

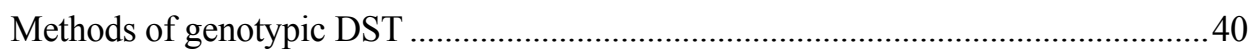

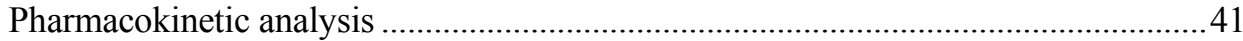

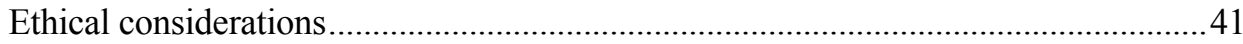

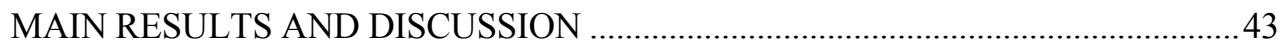

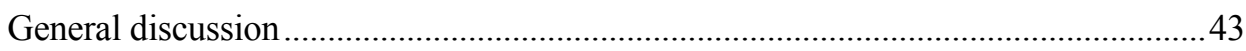

The importance of reliable drug susceptibility testing ..............................................44

Can measurement of drug concentrations be a useful tool in TB treatment?..............52

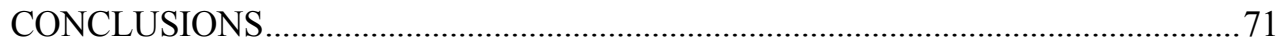

FUTURE PERSPECTIVES ON INDIVIDUALISED TREATMENT OF

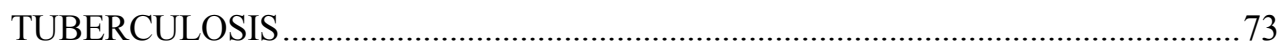

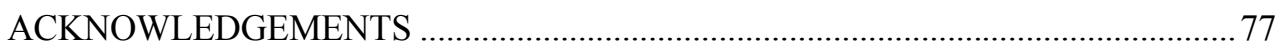

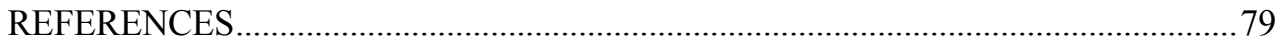





\section{ABSTRACT}

Each year, around 10 million of individuals develop active tuberculosis (TB). Worldwide, TB is the leading cause of death from an infectious agent surpassing both malaria and HIV. Current treatment regimens are long and therefore encompass a risk of nonadherence and development of acquired drug-resistance, reflected in the increase of multidrug-resistant (MDR) and extensively drug-resistant (XDR) TB. Indeed, this calls for prudent use of existing TB drugs and improvement of TB treatment strategies. The aim of this thesis was to investigate the current drug susceptibility testing (DST) breakpoints for Mycobacterium tuberculosis (M. tuberculosis), the pharmacokinetics and pharmacodynamics (PK/PD) of TB treatment and to explore the role of therapeutic drug monitoring (TDM) for optimising TB treatment.

Drug resistance in $M$. tuberculosis is expressed over a continuous scale and for some drugs it may be identified as low- and high-level resistance. This has been poorly reflected in currently used binary susceptibility breakpoints for TB drugs. Results from genome sequencing and phenotypic DST of ofloxacin and levofloxacin were compared in study I and current breakpoints were found to misclassify up to $25 \%$ of $M$. tuberculosis isolates with resistance mutations in $g y r A$ as susceptible to fluoroquinolones. This finding may have implications for the classification of XDR-TB, treatment of MDR-TB and the evaluation of fluoroquinolones in clinical studies.

Study II was a prospective cohort study of susceptible TB in Sweden, where drug concentrations of first-line TB drugs were measured along with the susceptibility level of the bacteria defined by the minimum inhibitory concentration (MIC) of M. tuberculosis. First-line drug concentrations below the reference range (16-42\%) were common and most pronounced for rifampicin $(13 / 31,42 \%)$. An exploratory investigation of PK/PD parameters displayed a wide distribution of ratios between drug exposures and MICs. Rifampicin exhibited higher level of individual fluctuations over time during TB treatment compared with isoniazid. In study III the plasma drug concentrations of rifampicin were compared to the tuberculosis drug activity assay (TDA) and results showed that rifampicin drug levels, but not drug levels of the other first-line drugs, correlated with TDA. Patients with rifampicin drug levels below $8 \mathrm{mg} / \mathrm{L}$ had significantly lower median TDA. This finding supports the use of TDA as a potential indicator for low rifampicin exposure in resource-constrained settings without access to drug concentration analysis. The study design in study II has been further developed in study IV, which is a prospective cohort study of MDR-TB in China, where drug exposure will be explored in relation to individual bacterial MIC and measurements of treatment outcome.

In summary, the work in this thesis emphasises the importance of reliable DST of $M$. tuberculosis and the need to re-evaluate the currently used breakpoints. Therapeutic drug monitoring (TDM) based on drug concentrations and MICs is a useful tool to avoid suboptimal drug exposure and to individualise TB treatments. Such strategies may improve treatment regimens and avoid further development of resistance. 



\section{POPULÄRVETENSKAPLIG SAMMANFATTNING}

Tuberkulos är en luftburen infektionssjukdom, som vanligen orsakas av tuberkelbakterien Mycobacterium tuberculosis (M. tuberculosis). Lungtuberkulos, som förr ofta kallades lungsot, är den vanligaste tuberkulosformen och orsakar symtom såsom långvarig hosta, nattsvettningar och avmagring. Tack vare förbättrad levnadsstandard och introduktion av tuberkulosläkemedel på 1950-talet, har antalet tuberkulosfall sedan dess minskat drastiskt i många länder. I Sverige har förekomsten av tuberkulos sedan 1940 sjunkit från 300 till ungefär 5 fall per 100000 invånare idag. Så ser det dessvärre inte ut överallt i världen; flera länder i Asien och många länder i Afrika söder om Sahara har fortfarande en tuberkulosförekomst som liknar den som Sverige hade 1940. Trots att infektionen sedan länge är botbar och numera en ovanlig dödsorsak i Sverige, så är tuberkulos globalt sett den infektionssjukdom som skördar flest liv, fler än malaria och HIV/AIDS. Orsakerna till detta är flera, såsom bristfällig sjukvård, ogynnsam samsjuklighet med HIV/AIDS samt ökning av mer svårbehandlad tuberkulos med motståndskraft mot de mest effektiva läkemedlen, så kallad resistens.

Tuberkulosbakterien utvecklar lätt antibiotikaresistens och behandlingen behöver alltid bestå av flera antibiotika samt pågå under lång tid för att undvika återfall. Känslig tuberkulos, utan antibiotikaresistens, behandlas i minst sex månader med kärnpreparaten rifampicin och isoniazid tillsammans med pyrazinamid och ethambutol de första två månaderna. Multiresistent tuberkulos (MDR-TB) innebär att tuberkulosstammen uppvisar resistens mot kärnpreparaten och behandlas i upp till två år. Behandling av MDR-TB består av ännu fler läkemedel och de mediciner som står till buds är generellt mindre effektiva och ger ofta svåra biverkningar. Uttalat resistent tuberkulos (XDR-TB) innebär utöver resistens mot rifampicin och isoniazid även resistens mot aminoglykosider och kinoloner, där den sista läkemedelsgruppen utgör hörnpelaren i MDR-TB behandling. Globalt är utläkningen av känslig tuberkulos strax över $80 \%$ och vid MDRoch XDR-TB 55\% respektive 34\%. God tillgång till tillförlitlig metodik för resistensbestämning är avgörande för att rätt tuberkulosbehandling ges. För att uppnå Världshälsoorganisationens mål om att minska förekomsten av tuberkulos till 2030 kan vi inte ur ett behandlingsperspektiv bara förlita oss på nya läkemedel, utan nuvarande behandlingar behöver förbättras och kortas.

Det övergripande syftet med avhandlingen är att undersöka möjligheter till optimerad och individualiserad behandling av tuberkulos och baseras på fyra studier.

Studie I undersöker huruvida viktiga brytpunkter, som i laboratoriet används för resistensbestämning av tuberkelbakterien, på ett säkert sätt kan skilja mellan känslig och resistent tuberkulos. I studie I genomgick 75 huvudsakligen antibiotikaresistenta bakteriestammar, varav många var av typen MDR/XDR-TB, genetisk analys samt odlades på plattor för odlingsbaserad resistensbestämning mot kinolonerna ofloxacin och levofloxacin. Vidare analys visade att dagens brytpunkter för ofloxacin och levofloxacin kan klassificera upp till 25\% av bakteriestammar som känsliga för dessa läkemedel trots att de är genetisk resistenta. Detta kan påverka den behandling som ges vid MDR-TB och klassificeringen av XDR-TB. 
Studier av förhållandet mellan läkemedelskoncentrationer i blod och bakteriens nivå av antibiotikakänslighet i relation till behandlingsutfall utfördes i studie II vid behandling av känslig tuberkulos i Sverige och i studie IV vid behandling av MDR-TB i Kina. Låga koncentrationer i blod av tuberkulosläkemedel är vanligt förekommande bland tuberkulospatienter och kan ha många olika förklaringar. Flera studier, men långtifrån alla, har visat att låga koncentrationer av tuberkulosläkemedel ökar risken för sämre behandlingsutfall och resistensutveckling. Motstridiga forskningsresultat kan bero på att de normalvärden för läkemedelskoncentrationer som används inte är väl utvärderade mot behandlingsutfall och att de inte tar hänsyn till graden av antibiotikakänslighet hos bakterien. I studie II inkluderades 31 vuxna patienter med känslig tuberkulos och mätning av läkemedelskoncentrationer utfördes vid flera tillfällen utöver resistensbestämning av patientens bakteriestam. Trots att patienterna fick rekommenderade läkemedelsdoser var för låga toppkoncentrationer vanligt (16-42\%) och i synnerhet var det så för det viktigaste läkemedlet rifampicin (13 av 31 patienter, $42 \%$ ). Låga nivåer av rifampicin i blod var i högre grad kopplat till svårare tuberkulossjukdom. Nyligen genomförda studier indikerar att högre doser av rifampicin än de som rekommenderas idag förbättrar behandlingseffekten varför behandlande läkare bör vara särskilt uppmärksam på låga nivåer av rifampicin. I studie III sågs att den tuberkelbakteriedödande effekten av blodplasma från patient som nyss tagit sin tuberkulosbehandling stämde väl överens med den läkemedelsnivån av rifampicin som kunde uppmätas i blodprovet med farmakologiska metoder. För övriga tuberkulosläkemedel (isoniazid, pyrazinamid och ethambutol) sågs inte detta samband. Fyndet stödjer att i länder som saknar utrustning för koncentrationsbestämning av läkemedel, kan ett blodprov tas för att istället testa grad av tuberkelbakteriedödande effekt, som ett mått på läkemedelsnivån i blod av det viktigaste läkemedlet rifampicin. Dosjusteringar baserat på mätning av läkemedelskoncentrationer i relation till bakteriens grad av antibiotikakänslighet förväntas kunna vara av ännu större vikt vid behandling av MDR-TB varför studie IV med liknande upplägg som i studie II har initierats i Kina där resultat väntas under 2019.

Sammanfattningsvis är det viktigt med tillförlitlig resistensbestämning av M. tuberculosis så att både klassificering av sjukdomen och behandling blir rätt. Mätning av läkemedelskoncentrationer kan vara ett användbart verktyg för behandlande läkare att upptäcka för låga blodnivåer av tuberkulosläkemedel. Om läkemedelsnivån i blod relateras till bakteriens antibiotikakänslighet och tuberkulossjukdomens svårighetsgrad vägs in, kan en bättre helhetsbild erhållas som kan ligga till grund för individuell dosjustering med mål att optimera tuberkulosbehandlingen. 


\section{LIST OF SCIENTIFIC PAPERS}

This thesis is based on the following publications, referred to in the text by their Roman numerals.

I Niward K, Ängeby K, Chryssanthou E, Paues J, Bruchfeld J, Jureen P, Giske CG, Kahlmeter G, Schön T. Susceptibility testing breakpoints for Mycobacterium tuberculosis categorize isolates with resistance mutations in gyrA as susceptible to fluoroquinolones: implications for MDR-TB treatment and the definition of XDR-TB.

J Antimicrobial Chemotherapy. 2016;71(2):333-8

II Niward K, Davies Forsman L, Bruchfeld J, Chryssanthou E, Carlström O, Alomari T, Carlsson B, Pohanka A, Mansjö M, Jonsson Nordvall M, Johansson AG, Eliasson E, Werngren J, Paues J, Simonsson USH, Schön T. Distribution of plasma concentrations of first-line anti-TB drugs and individual MICs: a prospective cohort study in a low endemic setting.

J Antimicrobial Chemotherapy. 2018;73(10):2838-45

III Niward K, Ek Blom L, Davies Forsman L, Bruchfeld J, Eliasson E, Schön T, Chryssanthou E, Paues J. Plasma Levels of Rifampin Correlate with the Tuberculosis Drug Activity Assay.

Antimicrobial Agents and Chemotherapy. 2018;62(5):e00218-18

IV Davies Forsman L, Niward K, Hu Y, Zheng R, Zheng X, Ke R, Cai W, Hong C, Li Y, Gao Y, Werngren J, Paues J, Kuhlin J, Simonsson USH, Eliasson E, Alffenaar JW, Mansjö M, Hoffner S, Xu B, Schön T, Bruchfeld J. Plasma concentrations of second-line antituberculosis drugs in relation to minimum inhibitory concentrations in multidrug-resistant tuberculosis patients in China: a study protocol of a prospective observational cohort study.

BMJ Open. 2018;8:e23899 



\section{ABBREVIATIONS}

AUC

CFU

$\mathrm{C}_{\max }$

$\mathrm{C}_{2 \mathrm{~h}}$

CRyPTIC

DOT

DST

EBA

ECOFF

EMB

FQ

gDST

HIV

$\mathrm{INH}$

LJ

LPA

MDR-TB

MGIT

M. tuberculosis

PanACEA

PCR

pDST

PK/PD

PZA

RIF

TB

TDA

TDM
Area under the concentration versus time curve

Colony-forming units

Maximum (or peak) drug concentration

Plasma drug concentration $2 \mathrm{~h}$ after drug intake

Comprehensive Resistance Prediction for Tuberculosis:

an International Consortium

Directly observed therapy for TB

Drug susceptibility testing

Early bactericidal activity

Epidemiological cut-off

Ethambutol

Fluoroquinolone

Genotypic drug susceptibility testing

Human immunodeficiency virus

Isoniazid

Löwenstein-Jensen

Line-probe assay

Multidrug-resistant tuberculosis

Mycobacterium Growth Indicator Tube

Mycobacterium tuberculosis

Pan-African Consortium for the Evaluation of Anti-

Tuberculosis Antibiotics

Polymerase chain reaction

Phenotypic drug susceptibility testing

Pharmacokinetics/Pharmacodynamics

Pyrazinamide

Rifampicin

Tuberculosis

Tuberculosis drug activity assay

Therapeutic Drug Monitoring 
Time to positivity (detectable growth of $M$. tuberculosis in liquid culture)

WGS

Whole genome sequencing

WHO

World Health Organization

XDR-TB

Extensively drug-resistant tuberculosis 


\section{PERSONAL PREFACE}

\section{A glimpse of my family's experience of tuberculosis during the pre-antibiotic era in Sweden:}

In my grandmother Ingeborg's village there was a 26 year-old woman living alone without any relatives and was suffering from severe end-stage pulmonary tuberculosis. No one in the society dared to help her out of fear being infected themselves. With her strong faith in God and endless will to help other people, my grandmother entered the sick woman's house and took care of her every day until she died. Later in life, my grandmother was tested with the tuberculin skin test and almost the entire arm turned swollen and red. My grandmother always told me that she would turn 100 years and during life she never developed active tuberculosis. My grandmother died of old age in 2012 at the age of 103.

"What counts is not the mere fact that we have lived. It is what difference we have made to the lives of others that will determine the significance of the life we lead."

Nelson Mandela 



\section{INTRODUCTION}

\section{Tuberculosis from a global perspective}

Tuberculosis (TB) caused by Mycobacterium tuberculosis (M. tuberculosis) is an air-born infectious disease traditionally associated with poverty, crowded living and malnutrition. History has proven that reduction of poverty through economic growth and increase of welfare through governmental reforms are efficient tools to reduce national TB incidence, even without access to medical treatment $(1,2)$.

Effective treatment of TB has been available since the early 1950's, but TB is still one of the top ten leading causes of death world-wide with a death toll in 2017 of 1.6 million people mainly affecting resource-limited countries (3). In 2017 there was an estimate of 10 million new cases of TB and the world-wide distribution is shown in Figure 1.

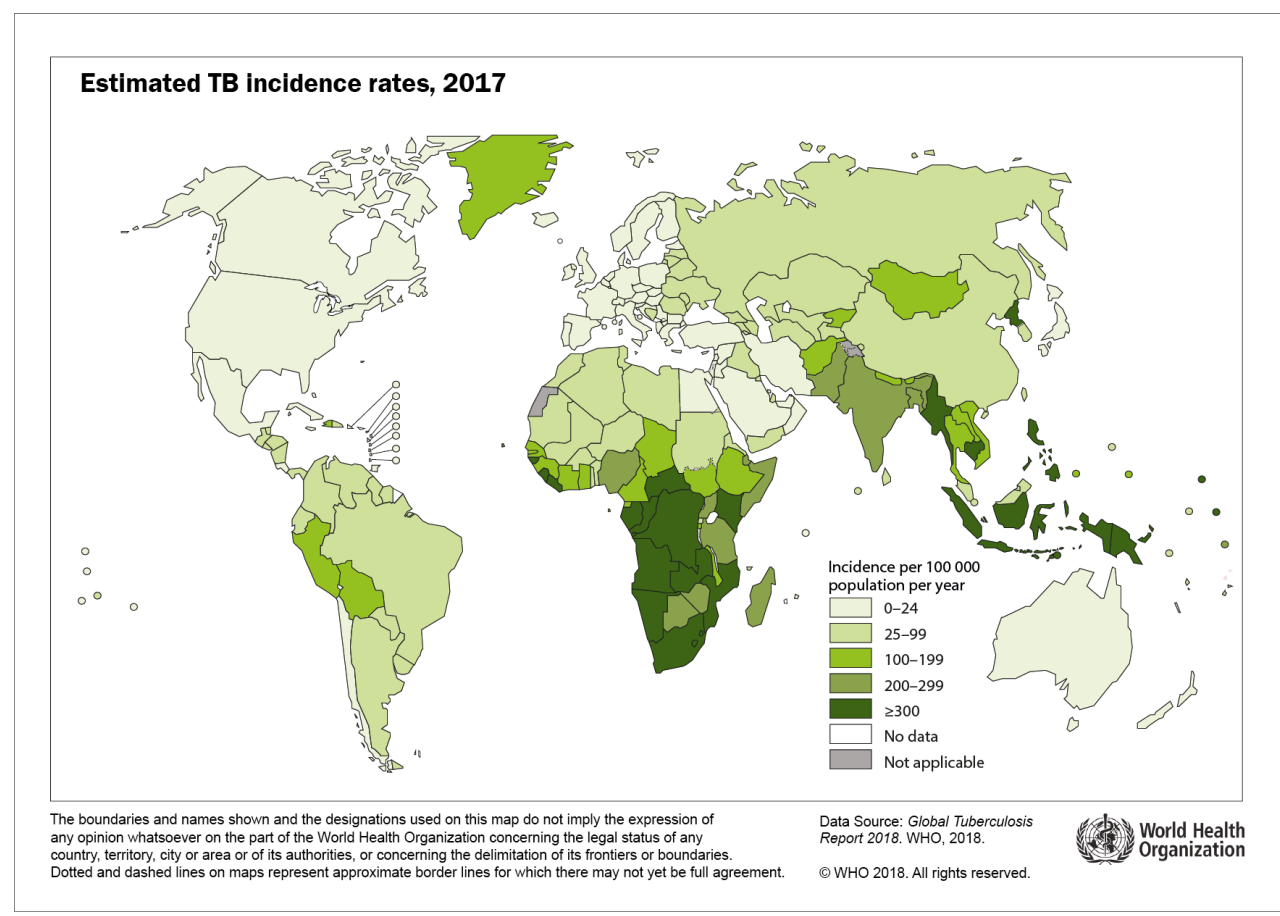

Figure 1. Global incidence of tuberculosis 2017. Reprinted with permission from the WHO.

Sweden had a TB incidence of 300/100 000 inhabitants in 1940, comparable to current incidence of TB in sub-Saharan Africa such as South Africa (Figure 1), but since then there has been a dramatic drop in TB incidence to approximately 5/100 000 inhabitants with 506 cases reported in 2018 (4). 
Even if the global total burden of TB that is susceptible to TB drugs slowly decreases, the proportion of TB resistant to key drugs so called rifampicin-resistant (RR), multidrugresistant (MDR) and extensively drug-resistant (XDR) TB is increasing, causing great suffering and mortality (Figure 2). The WHO estimated 558000 new cases of MDR/RRTB in 2017 whereof less than $30 \%$ were diagnosed or reported and only $25 \%$ were receiving adequate TB treatment. China and India alone accounted for $40 \%$ of this global gap (3). In Sweden 2,8\% (14/506) of reported TB cases in 2018 were MDR-TB and none was classified as XDR-TB (4).

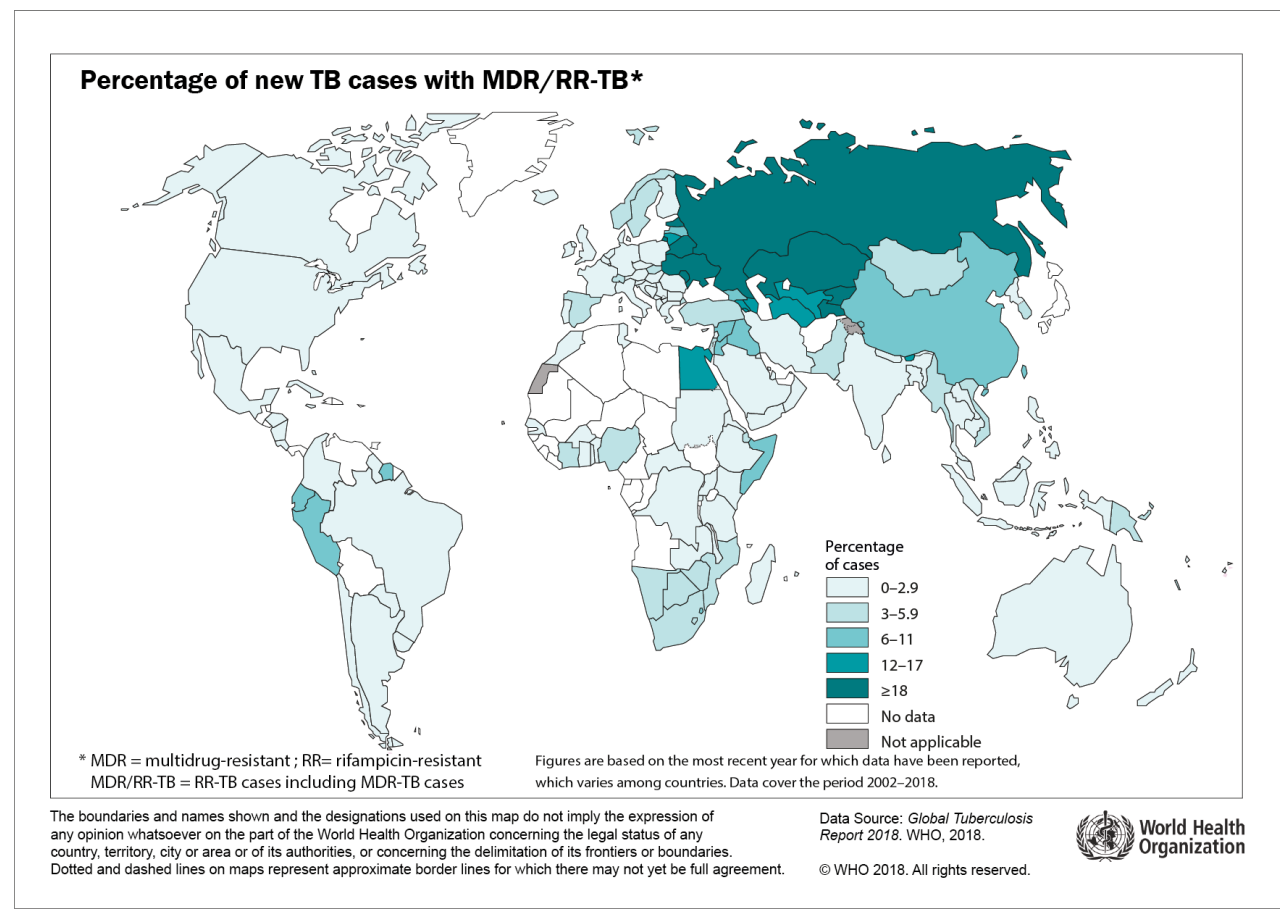

Figure 2. World-wide distribution of MDR-TB 2017. Reprinted with permission from the WHO.

Globally, treatment success rate for patients newly diagnosed with drug-susceptible TB is $82 \%$, and the corresponding rates for MDR- and XDR-TB are $55 \%$ and $34 \%$ respectively (3). Being very difficult to manage in terms of a long treatment duration often heavily compromised by side effects, MDR- and XDR-TB pose extreme challenges to clinicians, health care systems worldwide and for the patients. Antimicrobial resistance threatens to send us back to a time when we were unable to treat tuberculosis with other actions than nutrition, sunlight and collapse therapy (2) and calls for prudent use of antibiotics as one of several important interventions.

New anti-tuberculous (TB) drugs, such as bedaquiline and delamanid, are not the only solution to combat the TB epidemic bearing in mind that resistance has already emerged 
against these new drugs (5). Therefore, it is of paramount importance to use all TB drugs wisely and optimise current TB treatment along with socio-economic interventions in high-burden countries.

\section{Tuberculosis - basic concepts}

Tuberculosis is caused by genetically closely related members of the Mycobacterium tuberculosis complex, most frequently $M$. tuberculosis and more rarely other members such as M. africanum or the zoonotic M. bovis and M. microti $(2,6)$. Mycobacteria are in general aerobic, slow-growing intracellular bacilli and appear microscopically as acidfast rode-shaped (bacilliform) organisms (2). The bacilli are characterised by a complex thick lipid-rich cell wall, which mediates the bacilli resistance to drying, chemical disinfectants, therapeutic agents and the ability to survive inside macrophages $(6,7)$.

\section{Pathogenesis of tuberculosis}

Patients with pulmonary TB may primarily transmit the disease to close contacts, by cough-induced aerosol of infectious droplets. The outcome of TB infection is dependent on the host immune defence of the exposed individual. In a simplified model, there are three main outcomes after exposure - direct bacterial clearance by an effective innate immunity, latent TB in which the bacteria are controlled by cell mediated immunity and finally, primary progressive disease which may occur in patients with insufficient cell mediated immunity. After exposure, the innate immune response and host's phagocytic cells may effectively eliminate the bacilli in about $50 \%$ (8). In other contacts, primary infection occurs where the M. tuberculosis ensures its survival and replication in lung macrophages by mechanism such as inhibition of phagolysosomal fusion (9). By development of a cell-mediated host response (involving activated macrophages, $\mathrm{T}$ lymphocytes, tumour necrosis factor and interferon-gamma) further dissemination is prevented. Depending on the host immunity, reactivation to active TB disease may later occur. However, for the majority of individuals the primary infection is controlled by the cell mediated immunity and the bacteria persists in a life-long latent state. Latent TB is diagnosed as an asymptomatic individual with a positive tuberculin skin test or interferongamma release assay (IGRA) and a normal chest radiography (chest X-ray). Latent TB may reactivate within a few years after exposure but also years later with the highest risk among immunocompromised patients, such as patients with HIV/AIDS or on treatment with immunosuppressive drugs $(9,10)$. Latent TB represents a reservoir for TB as $5-10 \%$ may reactivate during their lifetime, and the WHO has estimated that one fourth of the world's population are carriers of M. tuberculosis. This reservoir is one of several challenges to reach the WHO's goal to end the TB epidemic by $2030(3,11)$.

In conclusion, the immune system is of paramount importance for TB control but since it may fail to eradicate slowly replicating bacilli or persisters, TB drugs with sterilising activity need to be included in TB treatment regimens (12-14). 


\section{Clinical symptoms and diagnosis of tuberculosis}

Patients with active TB present a wide range of manifestations and symptoms, therefore TB is often referred to as 'the big imitator' - a reminder not to forget the possibility of TB. Pulmonary TB is the most common clinical manifestation and extra-pulmonary manifestations often only account for $15-20 \%$ of active TB cases (3). Even though progression from latent TB to active disease may involve an asymptomatic subclinical phase with or without chest X-ray pathology, the inflammatory process commonly results in typical TB symptoms such as weakness, fever, loss of appetite, weight loss and night sweat. In case of pulmonary TB, the list of symptoms also includes chest pain, shortness of breath, and most prominently productive chronic cough which may be accompanied by haemoptysis $(6,9,15)$. A clinical suspicion of TB should initiate rapid examination and chest X-ray. Guided by the patient's symptom, other appropriate radiology and collection of specimens for detection and culture of mycobacteria should be performed. According to the $\mathrm{WHO}$, the case-definition of TB constitutes a patient with laboratory confirmed TB from a clinical specimen by smear microscopy, culture or a molecular diagnostic test or a case, in which a health care worker has diagnosed TB and decided to initiate TB treatment (16).

\section{Detection of M. tuberculosis}

Depending on the bacterial load in the sample, $M$. tuberculosis can be rapidly detected within hours by smear microscopy or polymerase chain reaction (PCR) based tests. Smear microscopy positivity requires more than 5,000-10,000 bacteria/ml for visual detection and a smear positive sputum indicates high infectiousness (17). When PCR-techniques were introduced in the 1990s for detection of M. tuberculosis the sensitivity for sputum samples, as compared with culture, greatly increased from at most about $50 \%$ with smear microscopy to $50-95 \%$ with PCR depending on which PCR-assay is used and on the bacillary load in the sample $(17,18)$. As an example the new rapid Xpert MTB/RIF Ultra, recently endorsed by the $\mathrm{WHO}$ as a screening tool for TB especially in high-endemic and resource-constrained areas (19), was described as having a sensitivity of $M$. tuberculosis complex detection in smear-positive sputum close to $100 \%$ and in smear-negative sputum around $65 \%$ as compared with culture (20). Hence, culture is still the most sensitive method for detection of M. tuberculosis, since the detection limit is just a few bacteria per millilitre. Solid egg-based media is the most commonly used substrate worldwide for culture (17). In many countries, a more expensive commercially automated system using liquid broth (BACTEC MGIT 960) has replaced the use of solid medium, since it reduces the mean turnaround time to detection to 1-2 weeks for smear positive samples, compared with 3 weeks on solid media (21).

\section{Principles of M. tuberculosis drug resistance}

In M. tuberculosis, the main mechanism of drug resistance is spontaneous mutations in chromosomal genes of growing bacteria (22). These mutations can cause drug resistance through overexpression or modification of drug targets (e.g. rifampicin and mutations in $r p o B$, isoniazid and $i n h A$, fluoroquinolones and $\operatorname{gyr} A(\operatorname{gyr} B)$, degrading/inactivating enzymes and loss of prodrug activation (e.g. isoniazid and mutations in katG, pyrazinamide and $p n c A)(22,23)$. Upregulation of efflux pumps commonly seen in Gram- 
negative bacteria, has been identified in $M$. tuberculosis for some of the first-line drugs and for bedaquiline (24-26).

In a population of $M$. tuberculosis, mutations occur naturally at a low rate and it was early observed that the risk of emergence of resistance was greater with increased bacillary load (27). Mutations resulting in drug resistance in vitro occur at different rate for each

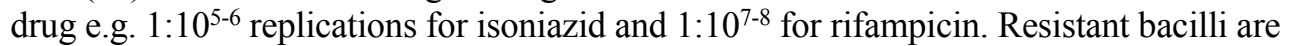
unlikely to be selected for multiplication if several active TB drugs with different mechanisms are given concomitantly (22).

Drug-resistant TB may be contracted in two ways, either by the in-host evolution of a resistant subpopulation of $M$. tuberculosis under selective pressure of suboptimal TB treatment (acquired or secondary drug-resistant $\mathrm{TB}$ ) or from direct transmission of drugresistant TB from one person to another (primary drug-resistant TB) (22).

\section{Tuberculosis treatment}

In the late 1940s, within a few years after the introduction of the first active drugs against TB (streptomycin and PAS), the link was discovered between pre-existing resistant mutants in the host before treatment initiation, selection of resistance during monotherapy, correlation between resistance and severe $\mathrm{TB}$, and the prevention of resistance in vitro by combining two drugs (27). Soon, the benefit of combined drug regimens for increased treatment efficacy and prevention of acquired resistance was proven in controlled clinical trials. Shortly after isoniazid was added to streptomycin and PAS in the 1950s, similar benefit of drug combinations was proven for isoniazid $(2,28)$. During the 1960s the most important sterilising agents, rifampicin and pyrazinamide, entered the market but since then no new TB drug classes have been developed or approved for drug-susceptible TB (29). However, after more than 40 years of waiting, bedaquiline and delamanid were introduced as potent agents approved for treatment of MDR/XDR-TB.

Tuberculosis treatment serves three main purposes; (i) to rapidly kill the large bacillary load multiplying in tissues (ii) to prevent emergence of clinically significant drugresistant mutants (iii) to effectively sterilise the site of infection (2).

\section{Treatment of tuberculosis susceptible to the first-line drugs}

Rifampicin revolutionised TB treatment when it was introduced as part of the combination therapy during the 1970s as it then was possible to reduce treatment length from 18 to 9 months. Adding pyrazinamide to the regimen allowed further shortening to six months, which remains the treatment length of drug-susceptible TB (29).

\section{First-line TB drugs}

First-line therapy is recommended for all new TB cases worldwide unless there are risk factors or drug susceptibility test (DST) pointing in the direction of drug-resistance. Treatment of fully drug-susceptible TB is based on an oral regimen and comprises an 
initial intensive two-month phase of four drugs; rifampicin, isoniazid, pyrazinamide and ethambutol, followed by a continuation phase consisting of rifampicin and isoniazid for four months. The optimal dosing frequency of oral first-line drugs is daily throughout the course of therapy, which also is recommended by the WHO (30). The mechanism of action is different for all first-line drugs and involves interference on both nuclei- and cell wall and cell membrane level of the bacteria as outlined in Figure 3, which in combination prevent development of drug resistance and relapse caused by persisters. 


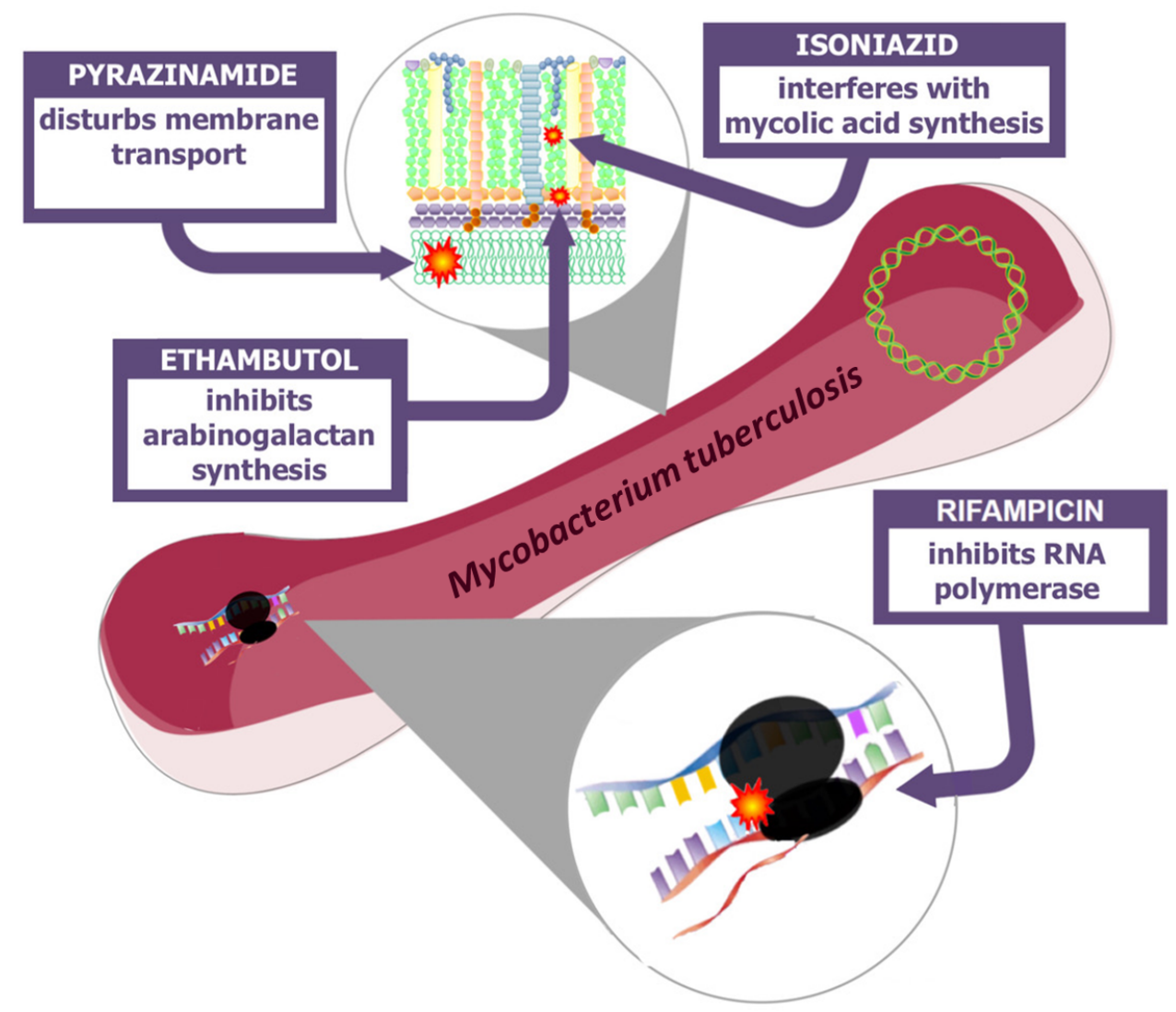

Courtesy of Sadaf Kalsum, Dep of Clinical and Experimental Medicine, Linköping University, Sweden.

Figure 3. Schematic overview of mechanism of action of first-line drugs. Rifampicin inhibits the $r p o B$ encoded $\beta$-subunit of DNA-dependent RNA polymerase of the mycobacteria and blocks the RNA synthesis $(31,32)$. Isoniazid is a prodrug that after passive diffusion through the cell wall is activated by the mycobacterial enzyme catalase-peroxidase katG. The activated drug inhibits an enzyme, encoded by the inhA gene, involved in cell wall mycolic acid synthesis (31, 33). Pyrazinamide is also a prodrug that is converted to pyrazinoic acid by mycobacterial pyrazinamidase, encoded by $p n c A$ gene. After being excreted to acidic environment it becomes active, and is believed to kill the bacteria by multiple mechanisms including disturbing cell membrane transport (34). Ethambutol inhibits arabinosyl transferase enzymes, encoded by embB, involved in cell wall synthesis (22). 
There are three postulated phases during treatment of cavitary pulmonary $\mathrm{TB}(2,35,36)$ which are commonly applied to illustrate the main action of the first-line drugs according to the drug properties and different growth-dependent features of subpopulations of $M$. tuberculosis (Table 1).

\section{Phase I - rapidly growing extra-cellular bacilli}

In phase I, the first days of treatment, rapidly multiplying extra-cellular tubercle bacilli in the cavity walls are quickly killed by isoniazid which is highly bactericidal and causes an initial approximately 10-fold drop of viable bacilli in sputum $(37,38)$. Even if isoniazid is active both intra- and extra-cellularly it has a comparatively lower activity during the continuing treatment period, when rifampicin and pyrazinamide are mainly responsible for the bacterial killing and sterilisation $(2,33,36)$.

\section{Phase II - slowly dividing extra-cellular bacilli in acidic environment}

Pyrazinamide is the least understood drug from a mechanistic point of view. It has little early bactericidal activity and is only active in an acidic environment. This makes pyrazinamide crucial for phase II, where surviving extra-cellularly bacilli in the lung lesions are killed more slowly but there is sufficient acute inflammation to generate local low $\mathrm{pH}$ necessary for the sterilising activity of pyrazinamide $(13,39)$. An academic riddle that has been the subject of debate for decades is whether the acidic conditions under which pyrazinamide works are intracellular within macrophages or extracellular, and even if more research is needed the accumulating data indicate the extracellular space (such as necrotic acellular parts of the cavity) to be the main site of action for this drug $(40,41)$.

\section{Phase III - sporadically multiplying intra-cellular persisting bacilli}

Rifampicin is a potent drug with activity both intra- and extracellular and during all three phases but is particularly crucial in the last phase. Rifampicin inhibits RNA transcription and protein synthesis, which occurs to some extent also in the stationary growth phases and makes this core drug critical to further sterilisation of M. tuberculosis $(2,36)$. Rifampicin has high lipid solubility and crosses cell membranes readily, thus easily penetrate M. tuberculosis infected macrophages (33). In phase III, suggested to last for about four months, sporadically multiplying intra-cellular bacilli are killed very slowly mainly by rifampicin and as inflammation often has subsided, there might be insufficient acidity for pyrazinamide action. 
Table 1. Recommended drugs for treatment of drug-susceptible TB. The drug regimen is designed to kill the different growth-dependent subpopulations of $M$. tuberculosis isolates actively dividing $(A)$, slowly growing $(B)$, and persisters $(C)$. For each subpopulation one drug is underscored and one is in brackets, the former indicates the most important drug for killing the population and the latter the least effective drug among those active on the population (2).

\begin{tabular}{|l|c|c|l|}
\hline \multicolumn{1}{|c|}{ Drug } & $\begin{array}{c}\text { Effect on } \\
\text { population A, } \\
\text { B and C }\end{array}$ & $\begin{array}{c}\text { PK/PD-parameter } \\
\text { best predictive } \\
\text { of activity (42) }\end{array}$ & \multicolumn{1}{|c|}{$\begin{array}{c}\text { Main adverse drug } \\
\text { reactions (21, 33) }\end{array}$} \\
\hline Rifampicin & $\mathrm{A}, \mathrm{B}, \underline{\mathrm{C}}$ & AUC/MIC $\sim \mathrm{C}_{\max } / \mathrm{MIC}$ & $\begin{array}{l}\text { Pruritus, rash, hepatotoxicity, } \\
\text { haematologic abnormalities }\end{array}$ \\
\hline Isonizid & $\underline{\mathrm{A}},(\mathrm{B}),(\mathrm{C})$ & AUC/MIC $>\mathrm{C}_{\max } / \mathrm{MIC}$ & $\begin{array}{l}\text { Polyneuropathy, hepatotoxicity, } \\
\text { hypersensitivity reactions }\end{array}$ \\
\hline Pyrazinamide & $\underline{\mathrm{B}}$ & AUC/MIC & $\begin{array}{l}\text { Hepatotoxicity, hyperuricemia, } \\
\text { arthralgia, rash }\end{array}$ \\
\hline Ethambutol & (A) & AUC/MIC & Optic neuritis \\
\hline
\end{tabular}

AUC: area under the time curve, $\mathrm{C}_{\text {max }}$ : maximum drug concentration, MIC: minimum inhibitory concentration, PK/PD: pharmacokinetics/pharmacodynamics

\section{Prevention of drug resistance during first-line treatment}

Isoniazid protects rifampicin against development of resistance during first-line therapy, since these two drugs are the main actors in killing the rapidly multiplying bacilli, amongst which the emergence of resistance is expected to happen. Inadequate isoniazid drug exposure has been related with rifamycin resistance in clinical studies on acquired drug resistance $(43,44)$, and using only first-line standard regimen for treatment of isoniazid-resistant TB increases the risk of the development of MDR-TB (45). Pyrazinamide seems to provide little protection against emergence of resistance $(12,36)$. In case of unknown isoniazid resistance from the start of treatment, ethambutol may serve to protect against development of rifampicin resistance, even though it has less activity against metabolically active extra-cellular bacilli and the least effective drug in the firstline regimen $(2,15)$. In settings where DST for all first-line drugs is available, ethambutol is usually removed from the regimen within the first month of treatment once drugsusceptible TB is confirmed by phenotypic testing.

First-line treatment of extra-pulmonary TB consists of the same regimen and often similar duration as used for pulmonary TB with the exception of involvement of central nervous system that usually is treated 12 months (15).

\section{Active treatment monitoring}

Treatment adherence is of paramount importance to avoid treatment failure and acquired drug resistance. When TB resurged during the 1980s, mainly due to the start of HIV epidemic, treatment adherence was under the spotlight as increasing TB incidence as well as an increased proportion of drug-resistant TB were noted (46). The WHO recommends 
to use directly observed therapy (DOT) to ensure adherence, most critical during the intensive phase, in second-line therapy and in treatment of certain vulnerable groups (15). Although, DOT has been a subject of much debate $(47,48)$, the clinical significance of DOT to reduce the risk of acquired rifampicin resistance has been shown in retrospective studies (46). Supervised treatment must be carried out in a context-specific and patientfriendly manner and recently the WHO launched guidelines for use of digital technologies such as video observed therapy (VOT) to support treatment adherence (49).

Monitoring of adverse events as part of the DOT package is of high importance during TB treatment, although first-line drugs are generally well tolerated (Table 1). Hepatotoxicity and skin reactions can sometimes require cessation of TB drugs. A stepwise reintroduction of drugs is then recommended starting with the drug least likely to have caused the adverse event $(15,33)$.

\section{Towards treatment optimisation of drug-susceptible TB}

Treatment of drug-susceptible TB has excellent clinical outcome, in some settings cure rates not far from $100 \%$ with very few relapses (35), but the treatment duration is still very long. So far, attempts to shorten treatment have been difficult. Although, a pooled analysis of patient-level data identified non-inferiority compared with standard treatment in four-month fluoroquinolone-containing regimens in participants with minimal noncavitary disease (50). Preclinical data have shown a near-linear dose-dependent bactericidal effect for rifampicin (51) and there is accumulating evidence that the current recommended dose of rifampicin $(8-12 \mathrm{mg} / \mathrm{kg})$ is suboptimal $(52,53)$. Out of five phase II studies, exploring higher doses or rifampicin for pulmonary TB, two studies failed to show significantly increased bacteriological response $(54,55)$. Three of the five studies were able to demonstrate more rapid bacteriological improvement, particularly with rifampicin doses of $35 \mathrm{mg} / \mathrm{kg}$, and with retained safety in the limited populations studied (56-58). In one of these studies (PanACEA MAMS-TB-01), the $35 \mathrm{mg} / \mathrm{kg}$ rifampicin arm experienced two weeks shorter time to sputum culture conversion in liquid media compared to standard doses of $10 \mathrm{mg} / \mathrm{kg}$ (58). No effect on final treatment outcome was reported for any of the studies exploring higher doses of rifampicin, although the studies did not have the power or primary aim to assess this outcome. A new study will be undertaken with the intention to shorten treatment, TRUNCATE TB (NCT03474198), where a two-month regimen will be compared with standard of care. The experimental arms include high-dose rifampicin $(35 \mathrm{mg} / \mathrm{kg})$ and a fifth drug added to the quadruple (linezolid, bedaquilin, levofloxacin or clofazimin). The ongoing RIFASHORT trail is exploring shorter treatment with $1200 \mathrm{mg}$ and $1800 \mathrm{mg}$ rifampicin (NCT02581527) and the potential benefit of the long half-life of rifapentine to shorten treatment is also investigated (NCT02410772).

Despite high cure rates of drug-susceptible TB, treatment failure does occur even in highresource, low-endemic settings. The nature of treatment failure in TB is multi-factorial and it is always important to exclude acquired drug resistance and never add a single drug to a failing therapy in order to avoid further emergence of resistance. 


\section{MDR TB treatment}

Drug-resistant TB is a man-made phenomenon due to acquired resistance emanating mainly from non-adherence to therapy, subtherapeutic drug concentrations at the site of the infection, incorrect prescription practices and inadequate health care delivery systems alone or in combination $(31,59)$. MDR-TB is defined as resistance to the first line key drugs (isoniazid and rifampicin). Pre-extensive drug-resistance (pre-XDR) means additional resistance to either fluoroquinolones (ofloxacin, levofloxacin, gatifloxacin and moxifloxacin) or second-line injectable drugs (amikacin, kanamycin or capreomycin). The definition of XDR-TB has so far been resistance to one of the fluoroquinolones and at least one of the three second-line injectable drugs in addition to MDR-TB (21). The definition of XDR-TB is likely to be revised following a recommendation by the WHO on downgrading of the second-line injectables and a more vital role for bedaquiline and linezolid together with fluoroquinolones in the treatment regimen of drug-resistant TB $(60,61)$.

Treatment of MDR-TB is challenging owing to the long duration of therapy (usually at least 18-20 months), use of in general less efficacious and more toxic drugs, suboptimal adherence, high costs and a low global cure rate of $55 \%(21,62)$. In a retrospective study on outcome data of MDR-TB patients treated in Sweden during 1992-2014 the treatment success rate was $83.5 \%(132 / 158)(63)$.

\section{Fluoroquinolones are important agents}

Fluoroquinolones act on the enzyme DNA gyrase thereby preventing bacterial DNA synthesis (33) with good in vivo and in vitro bactericidal activity against $M$. tuberculosis $(64,65)$. According to current WHO guidelines, the fluoroquinolones are among the most valuable second-line TB agents (62). This was further corroborated in a recent large metaanalysis on treatment correlates of successful outcomes in pulmonary MDR-TB showing significantly better treatment outcome including reduced mortality if levofloxacin or moxifloxacin was included in the regimen (66). Ofloxacin has been surpassed by more efficacious later-generation fluoroquinolones such as moxifloxacin, levofloxacin and gatifloxacin. Levofloxacin is recommended in combination regimens with rifampicin, since it does not interact with rifampicin as moxifloxacin does, with a rifampicin-induced up to $30 \%$ reduction of moxifloxacin drug exposure $(67,68)$. This drug-drug interaction is no concern in treatment of MDR/XDR-TB as rifampicin is excluded from the regimen. The use and availability of gatifloxacin has been compromised by concerns of observed dysglycaemia (62). Levofloxacin and moxifloxacin have excellent penetration to cerebrospinal fluid and several clinical trials have investigated the role of a fluoroquinolone as a first-line agent in treatment of TB meningitis (69). However, both long-term outcome, drug-doses, suitable dosing and safety aspects need further investigation. Moreover, moxifloxacin is part of the WHO endorsed short-course regimen for MDR-TB since levofloxacin has not yet been evaluated in such regimens $(60,69)$, albeit there is ongoing evaluation of high-dose levofloxacin as part of short-course MDR$\mathrm{TB}$ regimen (70). 
The short-course treatment for MDR-TB

MDR-TB patients without previous use of second-line drugs and no resistance against fluoroquinolones and aminoglycosides fulfil the criteria for short-course MDR-TB treatment consisting of 9(-12) months treatment. The initial phase, 4-6 months, includes seven drugs; high-dose moxifloxacin, amikacin, clofazimine, prothionamide, high-dose isoniazid, pyrazinamide and ethambutol, followed by five months of moxifloxacin, clofazimine, pyrazinamide and ethambutol $(60,62)$. Treatment success rates comparable of conventional longer MDR-TB treatment have recently been reported in the STREAM I trail (71), but the benefit of this regimen has been questioned in Eastern European settings due to widespread resistance to several of the drugs included $(72,73)$.

\section{Conventional longer MDR-TB treatment}

Conventional MDR-TB treatment can either be provided to the patient as a standardised treatment, often used in resource-constrained areas, or as a customised treatment guided by DST and contact history (62). Both the number of effective drugs and DST availability affect treatment success rates and these regimens are usually designed to include preferably at least 4-5 effective drugs according to current WHO guidelines (Table 2). Recently, new evidence of effective fully oral regimens prompted the WHO to change guidelines for treatment of MDR-TB (60). The changes partly stem from a large individual patient meta-analysis of treatment outcomes in pulmonary MDR-TB showing a pooled treatment success rate of $61 \%$. The most important drugs for treatment success and reduced death were the later-generation fluoroquinolones, bedaquiline and linezolid (66). 
Table 2. Recommended drugs for MDR-TB treatment. Adapted from the WHO update 2018 $(21,33,60)$.

\begin{tabular}{|c|c|c|c|}
\hline Group & Drug & $\begin{array}{l}\text { Mechanism of } \\
\text { action }\end{array}$ & $\begin{array}{l}\text { Main adverse drug } \\
\text { reactions }\end{array}$ \\
\hline \multirow{3}{*}{$\begin{array}{l}\text { A } \\
\text { (include all if } \\
\text { possible) }\end{array}$} & $\begin{array}{l}\text { Moxifloxacin } \\
\text { OR } \\
\text { Levofloxacin }\end{array}$ & $\begin{array}{l}\text { Inhibits DNA } \\
\text { gyrase }\end{array}$ & $\begin{array}{l}\text { Arthropathy, tendonitis, } \\
\text { QT-prolongation }\end{array}$ \\
\hline & Bedaquiline & $\begin{array}{l}\text { Inhibits ATP } \\
\text { synthase }\end{array}$ & $\begin{array}{l}\text { QT-prolongation, } \\
\text { hepatotoxicity }\end{array}$ \\
\hline & Linezolid & $\begin{array}{l}\text { Inhibits protein } \\
\text { synthesis }\end{array}$ & $\begin{array}{l}\text { Myelosuppression, } \\
\text { neuropathy }\end{array}$ \\
\hline \multirow{2}{*}{$\begin{array}{l}\text { B } \\
\text { (add both if } \\
\text { possible) }\end{array}$} & \multicolumn{2}{|c|}{ Clofazimine } & $\begin{array}{l}\text { Pruritus, rash, reversible } \\
\text { skin discolouration }\end{array}$ \\
\hline & \multicolumn{2}{|c|}{$\begin{array}{l}\text { Cycloserine OR } \\
\text { Terizidone }\end{array}$} & $\begin{array}{l}\text { Neurotoxicity, psychiatric } \\
\text { disturbances, neuropathy }\end{array}$ \\
\hline $\begin{array}{l}\text { C } \\
\text { (add-on if drugs } \\
\text { from Group A and B } \\
\text { cannot be used) }\end{array}$ & \multicolumn{3}{|c|}{$\begin{array}{c}\text { Ethambutol, Delamanid, Pyrazinamide, Imipenem-cilastatin OR } \\
\text { Meropenem/clavulanic acid, Amikacin, } \\
\text { Ethionamide/Prothionamide, } p \text {-aminosalicylic acid }\end{array}$} \\
\hline
\end{tabular}

DNA: deoxyribonucleic acid, ATP: adenosine triphosphate

\section{Towards further treatment optimisation}

MDR-TB drugs are generally less efficacious drugs and have more adverse drug reactions, as compared with the first-line drugs. Clinical studies on shorter oral and tolerable treatments are highly prioritised research. Encouragingly, several ongoing clinical trials address these issues and shorter, all-oral regimens are to be expected (73). Bedaquiline, a novel antibiotic with bactericidal activity, and recently upgraded by the WHO (Table 2) is increasingly used in treatment regimens of MDR/XDR-TB with significantly better outcomes, although QT-prolongation might limit its use $(3,66,73)$. Stage II of the STREAM trial (NCT02409290) is evaluating short-course regimens for MDR-TB whereof two contain bedaquiline and one is completely oral. The ZeNIX study (NCT03086486) is evaluating an oral six-month combination of bedaquiline, pretomanid and linezolid in patients with newly diagnosed XDR-TB or those who have failed on MDR-TB treatment. 


\section{Drug susceptibility testing of $M$. tuberculosis}

Drug susceptibility testing serves two main important purposes: (i) individual management of TB cases including both treatment guidance and preventive actions and (ii) surveillance of TB drug resistance $(74,75)$. The value of DST for $M$. tuberculosis to individualise TB treatment is well known and resistance to key drugs has been linked to worse treatment outcome (76).

Drug susceptibility testing of $M$. tuberculosis can be determined either by culture-based phenotypic methods (pDST) or by genotypic tests (gDST) based on detection of resistance mutations related to drug action (77). The former predicts both susceptibility and resistance whereas the latter is so far typically used as a rule in test (78). Recently, whole genome sequencing (WGS) showed promising performance for replacing pDST of first line drugs (79). Indirect DST is most common and performed by using pure cultured isolates meanwhile direct DST is performed promptly on the sample obtained from the patient mostly smear and/or PCR positive specimens (74), the latter rarely used for pDST. Nowadays, gDST methods reduce the time between obtaining the specimen from the patient and reading the susceptibility result until a complete indirect pDST report is available. Phenotypic DST detects resistance regardless of the mechanism or molecular basis and is currently regarded as the reference method for drug resistance detection of M. tuberculosis (59).

\section{Phenotypic drug susceptibility testing}

There are many different available methods for culture-based DST of $M$. tuberculosis but the most frequently used technique is the proportion method described in the early 1960's by Canetti (74). Through the years, the phenotypic DST based on indirect testing and the proportion method, traditionally performed on solid media, has been extensively used and when new methods were adopted, solid media methods were often used as a comparator $(21,59)$.

\section{The proportion method and definition of critical proportion}

The rationale for the proportion method is the small amount of pre-existing drug-resistant bacilli within the wild-type strains, whereas the resistant strains contained higher proportion of such bacilli. A cut-off between susceptible and resistant isolates at a critical proportion of $1 \%$ was selected from those studies $(27,74)$.

In the indirect $1 \%$ proportion method on solid medium the inoculum used is monitored by testing two dilutions of a culture suspension, and the growth (the number of colonies) on the drug-containing medium is compared with the growth present on the drug-free control medium. The strain is regarded as resistant if growth on the medium containing a defined drug concentration (i.e. the critical concentration) exceeds $1 \%$ of the inoculum $(59,80)$. The critical concentrations for the TB drugs were determined experimentally by comparing growth of clinical strains corresponding to wild-type and non-wild-type, and it was concluded that a reduced clinical response could be expected in non-wild-type strains above the critical concentration (81). Critical concentrations of TB drugs used for pDST varies with the medium used as this value depends on the drug concentration that 
remains active in the medium after completion of the preparation of the drug-containing solid or liquid media (75).

\section{Methods used for phenotypic DST}

Worldwide, the proportion method is most commonly used for pDST and the inexpensive Löwenstein-Jensen solid egg-based media represents the only available media in many high-endemic countries (59). In high-resource settings routine pDST is typically performed in BACTEC MGIT 960 system (Becton Dickinson, Franklin Lakes, NJ, USA) using a modified proportion method and liquid 7H9 Middlebrook media $(21,82)$. Table 3 shows an overview of common phenotypic methods used for pDST in M. tuberculosis. Reading of results in pDST performed on solid or in liquid media can either rely on direct evidence of growth (i.e. counting of colonies) or on an indirect measurement such as bacterial oxygen consumption and production of carbon dioxide which is used in BACTEC MGIT 960 (82).

Table 3. Overview of common methods used for phenotypic drug susceptibility testing in M. tuberculosis. Adapted from previously published review (75) and WHO guidelines (21).

\begin{tabular}{|l|l|l|}
\hline \multirow{2}{*}{ Method } & \multicolumn{2}{c|}{ Detection of growth } \\
\cline { 2 - 3 } & \multicolumn{1}{|c|}{ Direct } & \multicolumn{1}{c|}{ Indirect } \\
\hline Broth microdilution ${ }^{1}$ (e.g. Sensititre) & $\begin{array}{l}\text { Visible growth in broth- } \\
\text { wells by an inverted mirror }\end{array}$ & \\
\hline BACTEC MGIT 960 (7H9) & & Fluorometric test \\
\hline $\begin{array}{l}\text { Löwenstein-Jensen } \\
\text { (WHO and ECDC protocol) }\end{array}$ & $\begin{array}{l}\text { Visible colony growth on } \\
\text { slants }\end{array}$ & \\
\hline $7 \mathrm{H} 10^{3}$ or $7 \mathrm{H} 11^{3}$ (CLSI protocol) & $\begin{array}{l}\text { Visible colony growth on } \\
\text { plates }\end{array}$ & \\
\hline
\end{tabular}

${ }^{1}$ Endorsed by Clinical and Laboratory Standards Institute (CLSI) (83)

${ }^{2}$ Solid egg-based medium

${ }^{3}$ Solid agar-based Middlebrook medium

MGIT: Mycobacterial growth indicator tubes

\section{Determination of minimal inhibitory concentration}

Quantification of drug susceptibility can be performed by determination of the minimum inhibitory concentration (MIC), which is the lowest concentration of an antimicrobial agent that prevents visual growth. For M. tuberculosis, the MIC is often additionally defined according to the $1 \%$ proportion method rather than the visual growth. Consequently, the MIC will be the lowest drug concentration with an inhibition of more than $99 \%$ of the microorganisms in a solid or broth dilution susceptibility test (59). Instead of testing the susceptibility at one single predefined drug concentration, the method for MIC determination is based on two-fold serial dilutions of drug concentrations and typically form normally distributed bell-shaped curves for drugsusceptible strains as illustrated in Figure 4. Determination of MIC can be performed on 
solid media (e.g. LJ or Middlebrook 7H10/7H11) but more commonly liquid media, such as Middlebrook 7H9 in BACTEC MGIT 960, is used to reduce turnaround time.

Unlike other major bacterial pathogens there is no reference method established for MIC determination, indicating that there might be differences in how drug stock solutions and bacterial inoculum are prepared impairing comparability of data even on the same medium $(75,84)$. Compared to qualitative pDST based on single drug concentration this quantitative method is time-consuming but provides refined insight in the drug susceptibility level, although the inter- and intra-laboratory variability needs to be considered (84). Commercially available freeze-dried broth microdilution has recently been introduced for MIC determination, which enable susceptibility testing of multiple drugs simultaneously in 96 -well plates $(83,85)$. Although the concentration ranges for testing need to be adopted to avoid issues with truncated MICs, it is less expensive and labour-intense compared with BACTEC MGIT 960.

\section{Definition of drug susceptible and drug resistant TB}

The critical concentrations of TB drugs currently used for pDST of M. tuberculosis complex has been adopted and modified from international standard $(21,74)$. Recently, the definition of the critical concentration has been redefined by the WHO as the lowest concentration of a TB drug in vitro that inhibits growth of $99 \%$ ( $90 \%$ for pyrazinamide) of phenotypically wild-type strains of $M$. tuberculosis complex (59). A microorganism within the wild-type population is by definition devoid of phenotypically detectable resistance mechanisms against the drug in question. In contrast, non-wild-type organism harbour aquired or mutational resistance against the drug $(86,87)$. The range of MICs, typically 3-5 two-fold dilution steps, composing the wild-type population (Figure 4), is a consequence of intra- and inter-laboratory technical variation and biological differences in susceptibility among isolates (88). The epidemiological cut off (ECOFF) corresponds to the upper end of the wild-type distribution, i.e. it typically encompasses $99 \%$ of the phenotypically wild-type strains and therefore represents the highest MIC that still shows phenotypical drug susceptibility and is now equal to the critical concentration according to the latest definition $(59,87)$. The minimum aggregated data required for setting ECOFFs are 100 reported MIC values and within these there should be at least five valid MIC distributions generated in separate laboratories (87). 


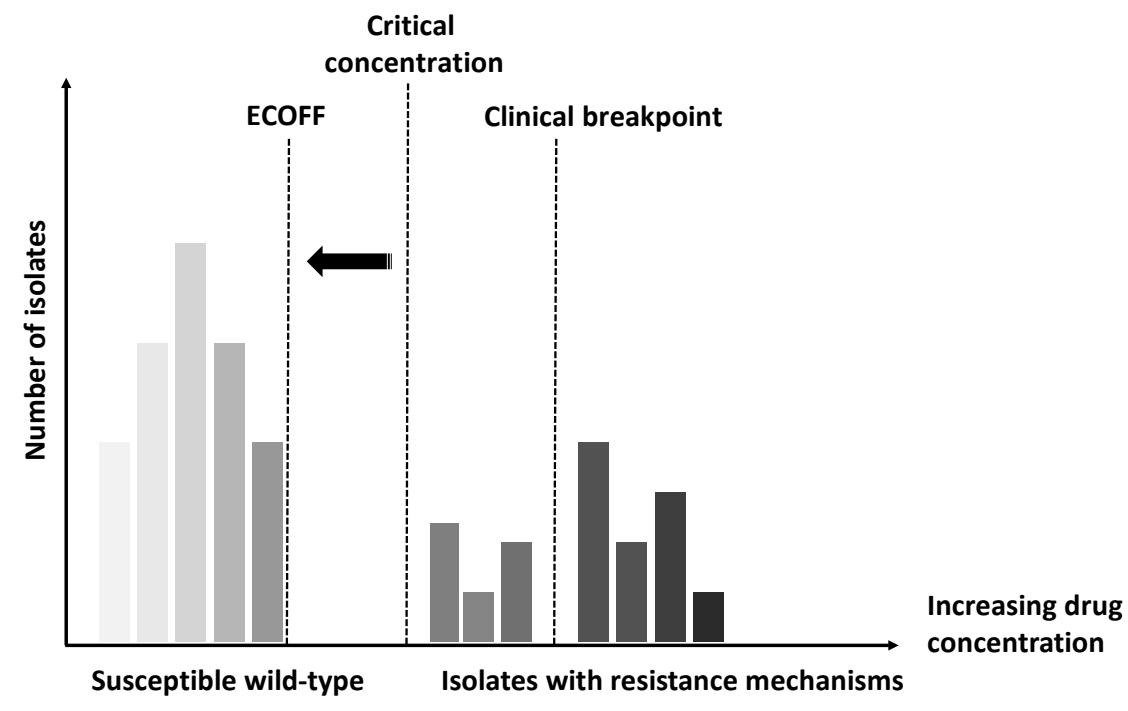

Figure 4. Schematic histogram of the relationship between ECOFF, critical concentration and clinical breakpoint. The critical concentration of TB drugs for M. tuberculosis, the ECOFF and the clinical breakpoint (if defined) often coincide. Recently, it was decided by the WHO that the definition of the critical concentration is the same as for the ECOFF. Eventually, when sufficient data are available, the critical concentration should be equal to the ECOFF (indicated by the arrow). A clinical breakpoint higher than the ECOFF may be set if PK/PD and/or clinical outcome data are in favour for a clinical efficacy at that resistant level. The number of MIC dilution steps has been increased for clarity.

It is important to understand that ECOFF alone does not by default categorise isolates into clinically susceptible or resistant but needs to be combined with the pharmacokinetic (PK) and pharmacodynamic (PD) properties of the drug as well as data on clinical outcome in order to define clinical breakpoints (Figure 4) (89). However, ECOFFs represent the lowest possible clinical breakpoint and in the case of $M$. tuberculosis, most often coincide with the clinical breakpoint.

\section{Setting clinical breakpoints for antimicrobial agents}

Clinical breakpoints are for everyday use in the clinical laboratory to guide clinical decisions in patient treatment (59). The major regulatory bodies for defining phenotypic drug resistance by setting breakpoints are the Clinical and Laboratory Institute (CLSI) and Food and Drug Administration (FDA) for the US and the European Committee on Antimicrobial Susceptibility Testing (EUCAST) on behalf of the European Medicines Agency (EMA). 
A clinical breakpoint is an MIC value that should separate strains likely to respond to treatment from strains with the likelihood to fail on treatment (89). Clinical breakpoints according to the SIR-system ( $\mathrm{S}=$ susceptible, $\mathrm{I}=$ intermediate or more appropriate "susceptible, increased exposure", R=resistant) set by EUCAST are based on three main important elements; (i) MIC wild-type distribution of the bacteria which determines the ECOFF; (ii) PK/PD aspects of the drug; (iii) data from studies on clinical outcome (78). The in vitro-based ECOFF is the most conservative breakpoint between susceptible and resistant strains and the clinical breakpoint may very well be higher than the ECOFF if there is evidence that strains with elevated MICs are still treatable either by standard (S), or if tolerated, increased (I) drug dosages.

The definitions of the susceptibility categories S, I and R have recently been changed by EUCAST in order to better communicate to the clinician the possibility of using increased drug exposure (Table 4).

Table 4. Definitions of susceptibility testing categories included in the SIR-system. Adapted from EUCAST guidance for DST (90).

\begin{tabular}{|c|l|}
\hline $\mathbf{S}$ & $\begin{array}{l}\text { "Susceptible, standard dosing regimen" } \\
\text { - applies when there is a high likelihood of therapeutic success using a } \\
\text { standard dosing regimen of the drug. }\end{array}$ \\
\hline $\mathbf{I}$ & $\begin{array}{l}\text { "Susceptible, increased exposure" } \\
\text { - applies when there is a high likelihood of therapeutic success because } \\
\text { drug exposure is increased by adjusting the dosing regimen or by its } \\
\text { concentration at the site of infection. }\end{array}$ \\
\hline $\mathbf{R}$ & $\begin{array}{l}\text { "Resistant" } \\
\text { - applies when there is a high likelihood of therapeutic failure even when } \\
\text { there is increased drug exposure. }\end{array}$ \\
\hline
\end{tabular}

It is important to avoid splitting the wild-type distribution when setting breakpoints since it has detrimental effect on pDST reproducibility and the closer the breakpoint is to the wild-type median MIC, the greater this effect will be (88).

\section{Breakpoints used for TB drugs}

There is a lack of systematic evaluation of clinical breakpoints for M. tuberculosis. Remarkably limited data are available to set clinical breakpoints according to standard procedures including ECOFFs in combination with clinical outcome and PK/PD data to classify breakpoints into the SIR-system $(59,86,91)$. Predefined single critical concentrations corresponding to tentative ECOFFs or derived from expert opinion for the different TB drugs, are therefore widely used in practice as clinical breakpoints $(21,59)$. Hence, the critical concentrations have not been evaluated by the rigorous strategy used for other major bacterial pathogens and fungi and many breakpoints have been questioned in recent years $(75,86)$. It should be noted that clinical outcome data of a single drug in 
relation to the MIC level or pDST result is very challenging to obtain as multi-drug therapy is mandatory in TB. Furthermore, for the second-line drugs used in management of drug-resistant TB, many different regimens are used and often customized based on the individual susceptibility pattern. Moreover, the definition of clinical breakpoints for TB drugs defined by the WHO (Figure 5) differs from the definition of clinical breakpoints set by EUCAST according to the SIR-system (Table 4).

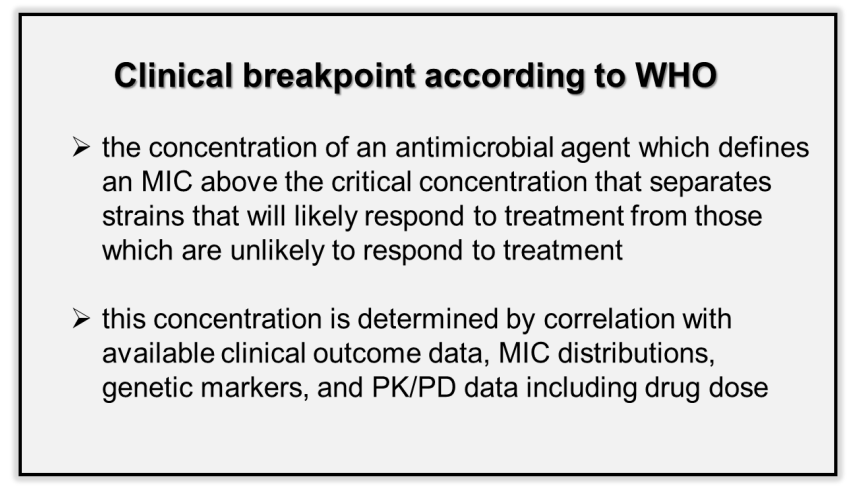

Figure 5. Definition of a clinical breakpoint for TB drugs according to the WHO (59). The clinical breakpoint is used to guide individual clinical decisions for patients on TB treatment.

The critical concentrations, first set by the WHO in the 1960s, are method-specific and have been regularly updated but evidence and methodology used to change breakpoints have so far not been documented. Albeit, inconsistencies in pDST results for $M$. tuberculosis have been acknowledged by the WHO and therefore a systematic review of MIC distributions in relation to resistance mechanisms was initiated in 2017. The purpose of the review was to assess the evidence for the different critical concentrations and clinical breakpoints used in pDST of second-line drugs. A similar systematic review of the critical concentration of first-line drugs will be undertaken during 2019 (59). The report presented merged analysis of data on MIC distributions (tentative ECOFFs were identified in a EUCAST fashion) in WHO endorsed pDST media. Additionally, resistance mutations were related to MIC levels and current critical concentrations. According to the definition, critical concentrations of TB drugs should ideally separate strains phenotypically wild-type from non-wild-type, but this is not always the case, as frequently observed in the technical report. Several breakpoint artefacts were unmasked and areas of insufficient evidence were identified concluding in that approximately 20 breakpoints were established, changed or withdrawn (59). Table 5 presents an overview of the currently used critical concentrations and (if defined) clinical breakpoints for the first-line drugs and for the fluoroquinolones, the latter a key second-line drug and subjected to several changes in the latest update. 
Table 5. Critical concentrations and clinical breakpoints used for first-line oral drugs and fluoroquinolones in different media and DST methods. Adapted from WHO guidelines (21) and technical report (59), in the latter a complete list of existing breakpoints for second-line drugs is available. Bold italic style represents changes of breakpoints according to the latest update 2018 and underlined breakpoints represent interim critical concentrations established despite very limited data. The first-line drugs are subject to revision during 2019.

\begin{tabular}{|c|c|c|c|c|c|}
\hline \multirow{2}{*}{ Drug group } & \multirow{2}{*}{ Drug } & \multicolumn{4}{|c|}{ DST critical concentrations (mg/L) } \\
\hline & & $\begin{array}{l}\text { Löwenstein- } \\
\text { Jensen }\end{array}$ & $\begin{array}{c}\text { Middlebrook } \\
7 \mathrm{H} 10\end{array}$ & $\begin{array}{c}\text { Middlebrook } \\
7 \mathrm{H} 11\end{array}$ & MGIT960 \\
\hline \multirow{4}{*}{$\begin{array}{l}\text { First-line } \\
\text { TB drugs }\end{array}$} & Rifampicin & 40.0 & 1.0 & 1.0 & 1.0 \\
\hline & Isoniazid & 0.2 & 0.2 & 0.2 & 0.1 \\
\hline & Pyrazinamide & - & - & - & 100.0 \\
\hline & Ethambutol & 2.0 & 5.0 & 7.5 & 5.0 \\
\hline \multirow{4}{*}{$\begin{array}{c}\text { Fluoro- } \\
\text { quinolones }\end{array}$} & Ofloxacin ${ }^{1}$ & 4.0 & 2.0 & 2.0 & 2.0 \\
\hline & Levofloxacin & $\underline{2.0}$ & 1.0 & - & 1.0 \\
\hline & $\begin{array}{l}\text { Moxifloxacin } \\
\text { Moxifloxacin } \mathrm{CB}^{2}\end{array}$ & $\frac{1.0}{-}$ & $\begin{array}{l}0.5 \\
2.0\end{array}$ & $\begin{array}{c}0.5 \\
-\end{array}$ & $\begin{array}{c}0.25 \\
1.0\end{array}$ \\
\hline & Gatifloxacin & $\underline{0.5}$ & - & - & $\underline{0.25}$ \\
\hline
\end{tabular}

${ }^{1}$ Ofloxacin DST is not recommended by the WHO as it is no longer used in TB treatment and laboratories are recommended transition to testing the specific fluoroquinolones used in the treatment regimens.

${ }^{2}$ Clinical breakpoints (CB) for 7H10 and MGIT apply to high-dose moxifloxacin (i.e. $800 \mathrm{mg}$ daily).

Several TB drugs exhibit a close relationship between the wild type and non-wild type MIC distribution. In such cases, the pDST is particularly prone to misclassifications when the currently predefined critical concentrations are used (such as for e.g. ethambutol and fluoroquinolones) and to poor pDST reproducibility due to the technical variability of up to \pm 1 two-fold dilution step in MIC testing. As this is a major pitfall detection of resistance mutations by gDST can elucidate whether isolates with MIC below but close to the critical concentration are wild-type or not i.e. the gDST result may overrule pDST (59, 92).

\section{The role of molecular biology in predicting resistance}

Culture of $M$. tuberculosis and pDST results take considerable time, often several weeks even in resource-rich countries where faster automated liquid broth systems are commonly used. Moreover, culture-based DST methods require advanced expensive laboratory infrastructure (biosafety level 3), well-educated staff and firm quality assurance mechanisms (82). If drug-resistant TB is not rapidly detected, use of inadequate antibiotics may lead to clinical failure and further spread of resistant bacteria as well as 
amplification of resistance. Thus, rapid diagnosis of drug-resistance is a prerequisite for appropriate treatment. Molecular DST methods use DNA sequences to detect resistanceconferring mutations and commercially available kits have a turnaround time down to 12 hours, but their diagnostic sensitivity is not perfect and consequently they can primarily rule in resistance, but not completely rule out (93).

\section{Methods used for rapid genotypic DST}

Two different PCR-based molecular methods are commercially available in clinical practice for rapid genotypic DST; Xpert MTB/RIF and line-probe assay (LPA). These gDSTs include, for core drugs, the most commonly known resistance mutations of $M$. tuberculosis associated with phenotypical resistance (Table 6).

The LPAs are expensive, require special laboratory facilities and are more labour-intense compared with Xpert MTB/RIF, but can still deliver rapid read-outs within two working days from a smear-positive patient. The latest version of Genotype ${ }^{\circledR}$ MTBDRsl assay detects specific mutations as well as other mutations (by absence of wild-type band) within genes associated with resistance to fluoroquinolones and aminoglycosides (94). Differences regarding targeted resistance genes between the two existing versions of the assay are delineated in Table 6 along with other commercially available LPAs such as Genotype ${ }^{\circledR}$ MTBDRplus for detection of resistance to the main first-line drugs. The performance of the different LPAs depends on the drug and if the test is used directly on clinical specimens or as an indirect test on culture. According to a large Cochrane review of studies using pDST and/or sequencing as reference standard the pooled sensitivity for MTBDRsl version 1.0 to detect fluoroquinolone resistance was around $86 \%$ with small differences between direct test on smear-positive specimens and indirect test (95). Moreover, if used on smear-positive specimens the assay detected $69 \%$ of individuals with XDR-TB and rarely gave a false positive result for individuals with TB infection without such resistance. The WHO has endorsed second-line LPAs as the initial test instead of pDST for patients with confirmed rifampicin-resistant TB or MDR-TB (94), but given the reduced sensitivity a pDST may be necessary if the LPA does not show resistance. 
Table 6. Overview of selected commercially available kits for gDST of $M$. tuberculosis. Adapted from ECDC TB laboratory guidelines (96). Within listed genes, specific target mutations are detected by the different assays as described elsewhere $(94,96,97)$.

\begin{tabular}{|c|c|c|c|c|c|c|c|}
\hline \multirow{3}{*}{ Method } & \multicolumn{7}{|c|}{ Overview of available kits for gDST } \\
\hline & \multirow[b]{2}{*}{ Assay } & \multirow{2}{*}{$\begin{array}{l}\text { Identification } \\
\text { of MTB } \\
\text { complex }\end{array}$} & \multicolumn{5}{|c|}{ Targeted resistance gene } \\
\hline & & & RIF & INH & Ag & FQ & EMB \\
\hline \multirow{2}{*}{$\begin{array}{l}\text { Real-time } \\
\text { PCR }\end{array}$} & Xpert $^{\circledR}$ MTB/RIF ${ }^{1}$ & yes & $r p o B$ & - & - & - & - \\
\hline & $\begin{array}{l}\text { Xpert }{ }^{\circledR} \text { MTB/RIF } \\
\text { Ultra }{ }^{1,2}\end{array}$ & yes & rроB & - & - & - & - \\
\hline \multirow{4}{*}{$\begin{array}{l}\text { Line } \\
\text { probe } \\
\text { assay }\end{array}$} & INNO-LiPA Rif & yes & rpoB & - & - & - & - \\
\hline & MTBDRplus V2.03,4 & yes & rроB & $\begin{array}{l}\operatorname{inh} A \\
\text { katG }\end{array}$ & - & - & - \\
\hline & MTBDRs/ V1.03 & yes & - & - & rrs & gyrA & $e m b B$ \\
\hline & MTBDRs/ V2.0 $0^{3,4}$ & yes & - & - & $\begin{array}{l}r r s \\
\text { eis }\end{array}$ & $\begin{array}{l}\text { gyrA } \\
\text { gyrB }\end{array}$ & - \\
\hline
\end{tabular}

${ }^{1}$ Assay with the Cepheid GeneXpert ${ }^{\circledR}$ System.

${ }^{2}$ Increased sensitivity for MTB detection compared to Xpert ${ }^{\circledR}$ MTB/RIF (20).

${ }^{3}$ The assay is part of the Hain Lifescience GenoType series.

${ }^{4}$ May according to the manufacturer, be performed on smear-negative sputum in contrast to the first version.

MTB: M. tuberculosis complex, RIF: rifampicin, INH: isoniazid, Ag: aminoglycoside, FQ: fluoroquinolone, EMB: ethambutol

\section{Towards transition to whole genome sequencing (WGS)}

The major pitfall with the rapid targeted molecular tests is, unlike the phenotypic tests, the failure to detect all existing drug resistance. In face of the strengths and limitations of both the rapid targeted gDST and the slow labour-intense pDST, the introduction of next generation sequencing is promising for rapid and comprehensive detection of drug resistance. Whole genome sequencing is a major step forward for molecular tests as it provides complete information of the nucleotide sequence of the organism's genome. The technique can, weeks faster than traditional diagnostic tools, produce simultaneous prediction of mycobacterial species, first- and second line drug resistance as well as provide strain typing for epidemiological purposes (98). Presently, WGS is only available in reference centres and is technical difficult to perform directly on clinical specimens, thus adding time for culture to the turnaround time of the method $(99,100)$. Even though WGS allows detection of all mutations conferring phenotypic resistance or susceptibility (101), interpretation of studies correlating genotypic and phenotypic resistance is influenced by the geographical distribution of resistance mutations as well as by the phenotypic method and breakpoints used as reference standard (102, 103). The relationship between mutations and phenotypic resistance is not completely known and 
the information obtained on genotype-phenotype presented in data-sharing platforms needs to be interpreted for clinical translation. There are now ongoing efforts to establish a comprehensive open-access database to enable user-friendly interpretation of WGS data for $\operatorname{TB}(93,104)$.

\section{Treatment outcome and surrogate markers of clinical improvement}

In clinical practice, it is often difficult to evaluate TB treatment particularly among patients with extra-pulmonary $\mathrm{TB}$ or culture negative $\mathrm{TB}$ as the main surrogate marker for response to TB therapy is smear- and culture-conversion, which is more of a microbiological outcome. The overall clinical features (weight gain, resolution or improvement of symptoms and/or radiology findings) are still important to support clinical decision-making $(15,21)$. Biomarkers can provide prognostic information and indicate normal or pathogenic processes as well as reflect response to therapy. In clinical trials of new drug regimens, early biomarkers such as changes in bacterial sputum load are often used as surrogate endpoints of treatment outcome. In particular for TB, improved surrogate markers for clinical outcome are urgently needed as the disease is associated with lengthy treatments and occurrence of delayed relapse (105).

\section{Sputum culture conversion and time to culture positivity}

The risk of relapse in pulmonary TB can be associated with some recognised predictors such as persistent sputum culture positivity in drug-susceptible TB at the end of the intensive phase i.e. after 8 weeks of treatment (15). For MDR-TB culture conversion is defined by two negative consecutive sputum cultures taken at least 30 days apart (21). However, studies have shown lack of consistency regarding delayed sputum culture conversion and risk of relapse $(105,106)$. A systematic review in 2010 of 35000 participants treated with standardised rifampicin-based regimens found both sputum smear microscopy and culture during treatment to have low sensitivity and modest specificity in predicting failure and relapse. The positive predictive values for these two entities were low (9-18\%) in predicting poor outcome suggesting low probability of a positive sputum specimen to correctly predict failure or relapse, but in contrast the negative predictive values were high (at least 93\%), indicating that a negative sputum test result makes relapse or failure unlikely (107). The WHO has previously recommended treatment extension in patients with a positive sputum smear after two months treatment but this is no longer part of the guidelines (30). However, a three months treatment extension for this group is included in many national guidelines in high-resource countries where sputum culture conversion is used as biomarker (108). In MDR-TB, sputum culture conversion at 6 months has shown to have higher sensitivity $(92 \%$ versus $27 \%$ ) but lower specificity ( $58 \%$ versus $90 \%$ ) in predicting treatment success compared with sputum culture conversion at two months (109).

In clinical studies, the effect of individual drugs can be evaluated by early bactericidal activity (EBA) represented by the decline in bacterial load during the first two weeks of treatment $(38,110)$. Sputum samples are collected at baseline and traditionally every 
second day after treatment initiation or with less rich sampling (e.g day $0,2,7,14$ ) and thereafter cultured on solid medium. The colony forming units (CFU) of each culture are manually counted and correspond to change over time in bacterial load. Not all drugs (e.g. clofazimine) exhibit an early EBA and are thus less suitable for evaluation by this method (111). Additionally, CFU counting in the TB laboratory is labour-intensive and time-consuming which makes it impossible to use in clinical routine. However, CFU can be monitored by measuring time to culture positivity (TTP) in sputum samples, which is automatically registered in the BACTEC MGIT $960(112,113)$ but rarely communicated to clinicians.

\section{Additional biomarkers and clinical severity score}

In addition to sputum culture conversion, chest X-ray provides useful information regarding disease extent and progress in pulmonary TB as well as being important for diagnosis of smear-negative cases in high-endemic settings. However, chest X-ray manifestations of pulmonary TB are very heterogeneous e.g. in adults versus children or immunocompetent versus immunocompromised and reporting is not standardised (114). Cavitary chest X-ray findings is well known to correlate with bacillary load (115) and the combined presence of cavitary disease and two-month positive smear/culture status was the best predictive marker of TB relapse in a recent systematic review (116).

The application of clinical scores to increase case-finding, evaluate treatment effect and enable risk assessment, has been developed for TB and evaluated in resource-limited areas. The Bandim TBscore is based on variables of signs and symptoms associated with TB chosen from the WHO guidelines (117). TBscore II is a simplified version (does not require a physician) of the original score validated in sub-Saharan Africa (118). It is graded from 0 to 8 points and based on the parameters presented in Figure 6. Previous studies have shown that the score has the ability to predict clinical outcome in TB patients in high-endemic settings (118). Less than a 25\% decline in TBscore II between treatment initiation and two-month follow-up predicted failure and mortality during the remaining part of the treatment. Further, failure was more prevalent in high TBscore II severity classes (III, IV). 


\section{TBscore II (0-8 p)}

\begin{tabular}{llll} 
Cough & $(1 p)$ & $B M \mid<18 \mathrm{~kg} / \mathrm{m}^{2}$ & $(1 \mathrm{p})$ \\
Dyspnea & $(1 p)$ & $B M \mid<16 \mathrm{~kg} / \mathrm{m}^{2}$ & $(1 \mathrm{p})$ \\
Chest pain & $(1 \mathrm{p})$ & MUAC $<220 \mathrm{~mm}$ & $(1 \mathrm{p})$ \\
Anaemia & $(1 \mathrm{p})$ & MUAC $<200 \mathrm{~mm}$ & $(1 \mathrm{p})$ \\
\hline
\end{tabular}

Figure 6. Clinical signs and symptoms included in TBscore II. Anaemia is estimated by visual check of pale inferior conjunctivae. Each of the four clinical variables contributes with one point, while body mass index (BMI) and measurement of mid upper arm circumference (MUAC) contribute an extra point if $<16 \mathrm{~kg} / \mathrm{m}^{2}$ and $<200 \mathrm{~mm}$, respectively, hence the maximum score of 8. Severity classes for TBscore II are; score $<2$ (I), score $2-3$ (II), score $4-7$ (III) and score $>7$ (IV).

\section{Pharmacokinetics and pharmacodynamics}

\section{Concepts of pharmacokinetics and pharmacodynamics}

Pharmacokinetics ( $\mathrm{PK}$, drug concentration over time) is the study of drugs (pharmaco) as they move (kinetics) through the body. Pharmacodynamics (PD, drug activity over time) describes what the drugs do to the body and the bacteria referred to as exposure-response relationship $(119,120)$.

\section{Pharmacokinetic parameters}

To understand the correlation between the administrated drug and the response produced, it is necessary to follow what happens with the drug within the body and over time. Pharmacokinetics is generally summarised by the measures absorption (A), distribution (D), metabolism (M) and excretion (E). Measurement of drug concentrations in plasma is a common strategy to gain knowledge of the internal drug exposure which depends on ADME. For several of the TB drugs the process is similar; the oral drug is absorbed into the systemic circulation, distributed throughout the body including to the site of TB disease, and eliminated by metabolism often in the liver and by excretion, either through the bile or the kidneys and released in the urine. Primary PK-parameters associated with ADME are listed in Table 7. Clearance and other primary PK-parameters are said to be linear when they are independent of dose or plasma concentration. Nonlinear kinetics occurs when one or more of the ADME are saturable i.e. the rate or extent of a process 
fails to increase in direct proportion to dose or plasma concentration e.g. a double dose results in more than expected increase in drug concentration $(120,121)$.

Table 7. Definition of key pharmacokinetic terms (120).

\begin{tabular}{|c|c|c|c|}
\hline Term & PK parameter & Definition & Factors of importance \\
\hline$\underset{\text { absorption }}{\text { A }}$ & $\begin{array}{c}\text { Oral } \\
\text { bioavailability } \\
(F)\end{array}$ & $\begin{array}{l}\text { The fraction of an } \\
\text { administrated drug } \\
\text { that reaches the } \\
\text { systemic } \\
\text { circulation, i.e. } \\
\text { from } 0-1(0-100 \%) \text {. }\end{array}$ & $\begin{array}{l}\text { Large, hydrophilic drugs are poorly } \\
\text { absorbed as opposed to small, } \\
\text { permeable lipophilic drugs. } \\
\text { Bioavailability can be decreased by } \\
\text { first-pass loss; decomposition and } \\
\text { metabolism in gut lumen, } \\
\text { metabolism and efflux-transporters in } \\
\text { the gut wall, metabolism in the liver. } \\
\text { The first-pass effect may be } \\
\text { saturable. Hence the bioavailability } \\
\text { will be dose-dependent. }\end{array}$ \\
\hline$\underset{\text { distribution }}{\text { D }}$ & $\begin{array}{c}\text { Volume of } \\
\text { distribution }\left(V_{d}\right)\end{array}$ & $\begin{array}{l}\text { The apparent } \\
\text { volume }(V) \text { the } \\
\text { drug is distributed } \\
\text { in and relates to } \\
\text { the ratio of the } \\
\text { amount of drug in } \\
\text { the body }(A) \text { and } \\
\text { plasma } \\
\text { concentration }(C) \text { : } \\
\qquad V=\frac{A}{C}\end{array}$ & $\begin{array}{l}\text { The theoretical value of } V \text { can vary } \\
\text { widely from } 3 \mathrm{~L} \text { to extensive tissue } \\
\text { distribution with values of } 40,000 \mathrm{~L} \\
\text { (e.g. drugs with high lipid solubility). } \\
\text { Plasma protein binding affects } \\
\text { volume of distribution where high } \\
\text { plasma protein binding gives low } V \text {. } \\
\text { Renal and liver disease can affect } \\
\text { protein binding. }\end{array}$ \\
\hline$\underset{\text { metabolism }}{\text { M }}$ & \multirow{2}{*}{ Clearance $(C L)$} & $\begin{array}{l}\text { The volume of fluid } \\
\text { that is cleared } \\
\text { from the drug per } \\
\text { unit of time (L/h or } \\
\mathrm{mL} / \mathrm{min}) \text {. }\end{array}$ & $\begin{array}{l}\text { Total clearance is the sum of all } \\
\text { pathways for drug elimination e.g. } \\
\text { the sum of hepatic clearance (mainly } \\
\text { by metabolism) and renal clearance } \\
\text { (mainly by excretion). }\end{array}$ \\
\hline $\begin{array}{c}\text { E } \\
\text { excretion }\end{array}$ & & $\begin{array}{l}\text { The clearance } \\
\text { determines the } \\
\text { plasma } \\
\text { concentration at } \\
\text { steady state. }\end{array}$ & $\begin{array}{l}\text { Hepatic clearance can increase by } \\
\text { induction of drug metabolising } \\
\text { enzymes in the liver (i.e. CYPs). } \\
\text { Renal clearance is decreased in } \\
\text { case of renal failure. }\end{array}$ \\
\hline
\end{tabular}

CYPs: cytochrome P450 enzymes

Secondary PK-parameters are derived from primary PK-parameters. Useful secondary PK-parameters are the drug half-life $\left(\mathrm{T}_{1 / 2}\right)$ reflecting the elimination kinetics and estimates of drug exposure such as the maximum drug concentration $\left(\mathrm{C}_{\max }\right)$, time to $\mathrm{C}_{\max }$ after drug intake $\left(\mathrm{T}_{\max }\right)$ and the area under the plasma drug concentration-time curve (AUC). Notably, it is only the free $(f)$ unbound drug concentration in plasma that mediates the drug activity therefore preferably $f \mathrm{C}_{\max }$ and $f \mathrm{AUC}$ should be used either by 
measuring free drug concentration in plasma or calculated by using known constants of protein binding (120).

Non-compartmental methodologies can be used for analysis of PK data based on manual calculations and the linear trapezoidal rule for measurement of AUC (Figure 7). This strategy gives approximate estimates of AUC during both the ascending and descending parts of the concentration-time curve and works well for drugs with linear elimination kinetics, i.e. plasma concentrations decline linearly over time. The AUC is normally calculated at steady state with a 24-hour time frame $\left(\mathrm{AUC}_{0-24 h}\right)(120,122)$.

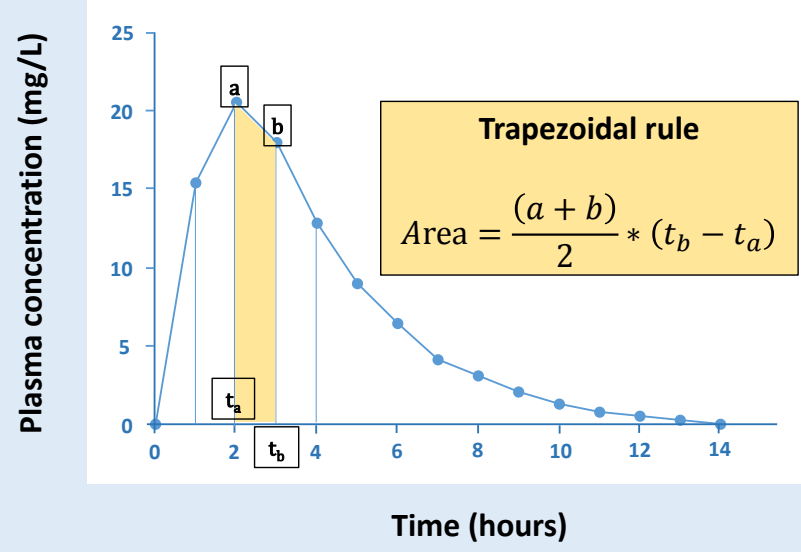

Figure 7. Graphical presentation of the assessment of the area under the plasma drug concentration versus time curve (AUC) by using the linear trapezoidal rule. The total $\mathrm{AUC}_{0-}$ $t$ is given by summarising the successive trapezoids between the individual measurements where the last measurement is defined as $t(120)$.

\section{PK/PD relationships}

In infectious diseases, the PD outcome is closely linked to the susceptibility level of the bacteria. The interaction of a drug with the microorganism can be described by the MIC and the kinetics of microbial inhibition or killing (119). This drug-microorganism interaction can be studied in vitro, in macrophage models and potentially in animal models and in human clinical studies. By integrating the knowledge of both PK and PD parameters of the specific drug, it is possible to identify the PK/PD indices most closely associated with effect (e.g. bacterial killing, suppression of drug resistance or clinical outcome), and the therapeutic range that gives high efficacy and low toxicity $(121,123)$. 
PK/PD indices used to describe the efficacy of antibacterial agents are either timedependent; (i) the cumulative percentage of a 24 hours period at steady state that a drug concentration remains above the MIC $\left(\% \mathrm{~T}_{>\mathrm{MIC}}\right)$, or concentration-dependent; (ii) the ratio of the peak drug concentration and the MIC $\left(\mathrm{C}_{\max } / \mathrm{MIC}\right)$, (iii) the ratio of $\mathrm{AUC}$ at the end of the dosing interval relative to the MIC (AUC/MIC) (124). There is often a close relationship between AUC/MIC and $\mathrm{C}_{\max } / \mathrm{MIC}$ (Figure 8) and these are the PK/PD indices that best predict activity for most of the TB drugs $(42,51,125-127)$ i.e. the optimal dosing regimens are those that maximise the drug concentrations.

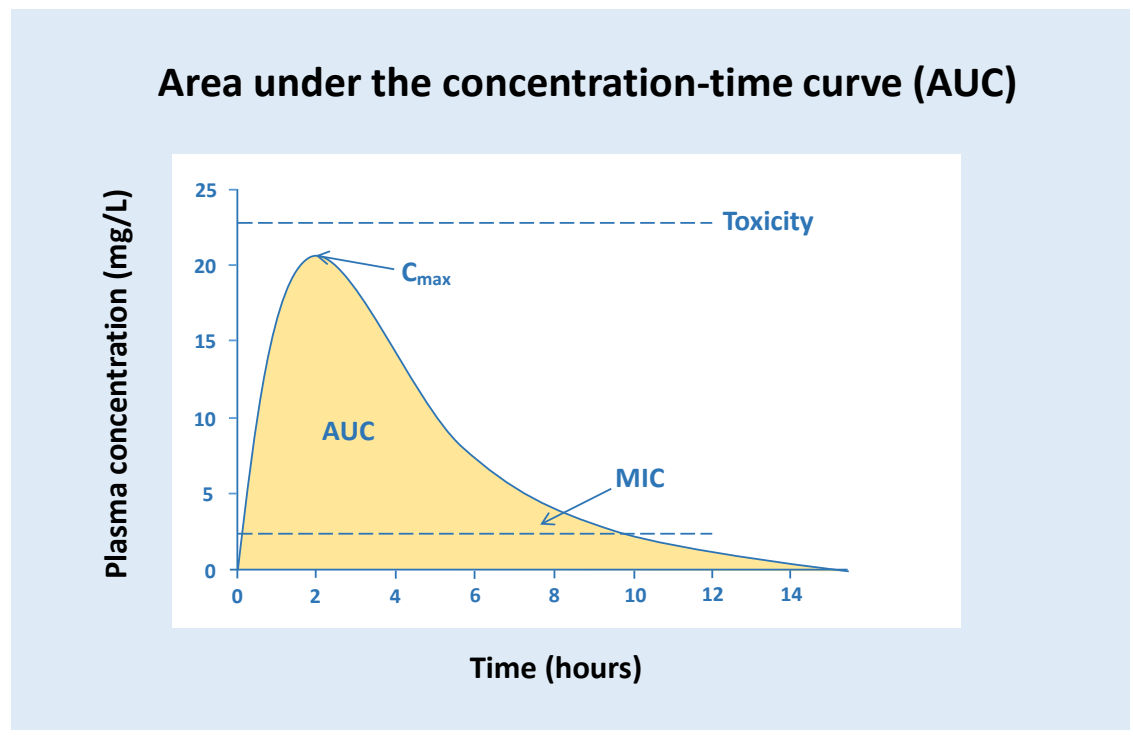

Figure 8. Schematic illustration of plasma-concentration-versus time curve, displaying the maximum plasma concentration $\left(\mathrm{C}_{\max }\right)$, the area under the curve (AUC) and the relationship of these parameters to the hypothetical MIC. The AUC is the area under the plot of measured plasma drug concentrations against time after drug administration. Drug toxicity (upper dashed line) is adverse effect in contrast to desired drug effect and this may occur at lower drug concentration level within the AUC.

In many clinical PK studies of TB treatment, there are not enough treatment failures to assess $\mathrm{PK} / \mathrm{PD}$ targets predictive of successful treatment as well as multidrug regimen is used, and therefore tentative targets for single drugs are often derived from preclinical studies (123). The hollow fibre system model has been increasingly used for PK/PD studies and is a more complex in vitro model. The aim of the hollow fibre system is to simulate human pharmacokinetics, and to study drug effects on different physiological or metabolic populations of $M$. tuberculosis. The PK/PD data retrieved may facilitate the design of optimal doses and dose schedules for the treatment of TB (128). 


\section{Measurement of drug concentrations}

Liquid-chromatography coupled with mass-spectrometry (LC-MS) is the routine technique for determination of plasma concentrations of antibiotics. The chromatography part (LC) is a powerful method for separation of components in a complex mixture where liquid solvents are forced through a column packed with solid adsorbent, with strong affinity for hydrophobic molecules. The compounds are thereby separated by their transition speed through the column which in turn depends on their degree of momentary binding to the adsorbent material. Once the compounds are chromatographically separated, they are detected by the mass-spectrometry (MS). Mass spectrometers operates by converting the analyte molecules retrieved from the LC to a charged (ionised) state, with subsequent analysis of their mass-to-charge ratio by using electro-magnetic fields for separation. The stream of the different ions passes through the MS for further separation and quantification electrically to produce the final output as a mass spectrum. Internal standards are pivotal for controlling day-to-day variability of the method (129).

\section{Therapeutic drug monitoring}

\section{The concept of therapeutic drug monitoring}

Therapeutic drug monitoring (TDM) is focused on improving patient care by measuring drug concentration and individual dose adjustment to optimise treatment efficacy and avoid concentration-dependent toxicity $(119,120)$. Thus, TDM rely on a definable relationship between dose and blood drug concentration, and between concentration and therapeutic effects. For optimal use of TDM, a therapeutic window needs to be defined with established targets for clinical efficacy. TDM is suitable for monitoring drugs with; narrow therapeutic windows, marked pharmacokinetic inter-individual variability, drug response that is difficult or slow to monitor, and for drugs known to cause therapeutic and adverse effects (130). Early examples of the application of TDM stretches back to the 1970s and involves drug monitoring of tricyclic antidepressants, digoxin, theophylline, and antiepileptic drugs but was also applied early in the field of infectious diseases by monitoring aminoglycosides (131). When TDM is used for individual based treatment in infectious diseases, the drug efficacy is dependent on the bacterial susceptibility. Therefore, drug concentration should, if possible, be related to the susceptibility level of the bacteria (Figure 9). 


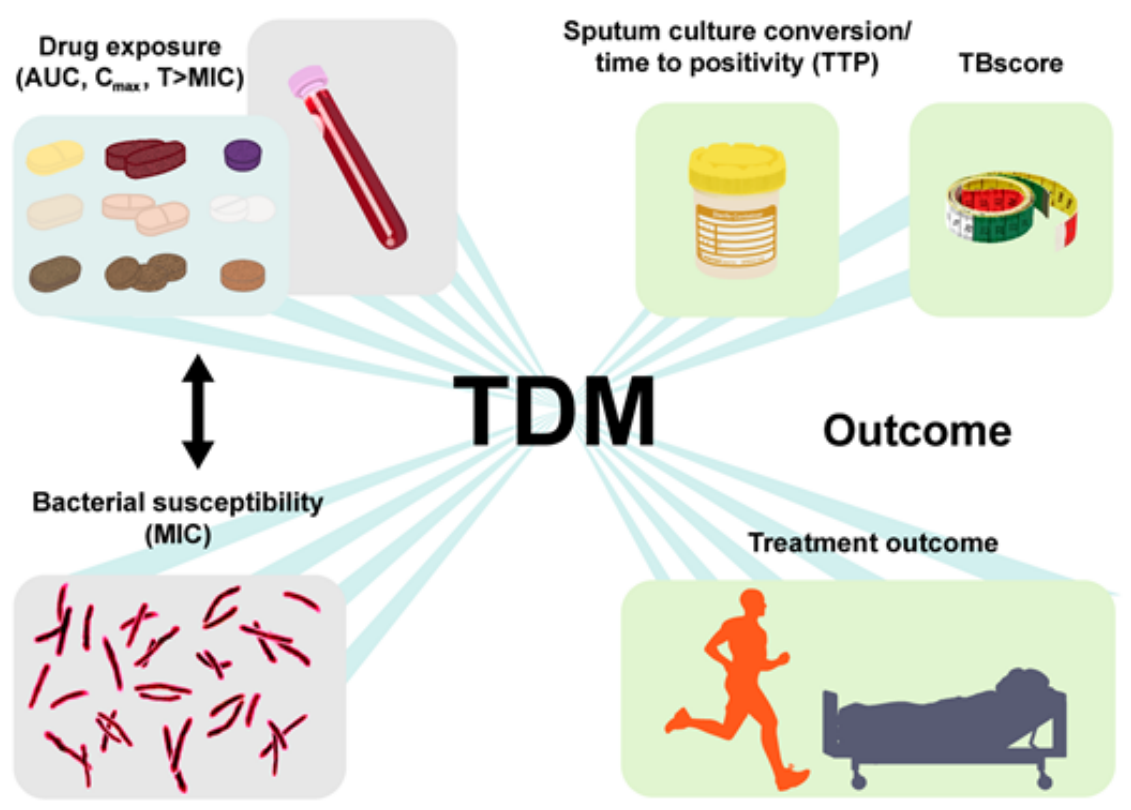

Figure 9. Therapeutic drug monitoring (TDM) - to correlate drug exposure to the susceptibility level of the bacteria in order to improve treatment outcome, avoid acquired drug resistance and minimise toxicity (illustration by Moi Peña 2018).

\section{The role of therapeutic drug monitoring in tuberculosis treatment}

Variability in drug exposure occurs as the result of inter-individual differences in the absorption, distribution, and elimination pathways of a drug. One dose does not result in identical concentration-time profiles in all individuals. The variability in PK distribution is affected by several factors such as the individual patient physiology, conditions with malabsorption or other comorbidities, drug-drug interaction or pharmacogenetics i.e. gene polymorphism in drug-metabolising enzymes. In addition, factors affecting the actual dose entering the body such as drug formulations, quality of tablets and adherence pattern influence PK-variability and has to be taken into account $(120,132,133)$. PK variability of TB drugs is common and has been identified as an important factor causing slow treatment response and development of drug resistance (42, 44, 127). A variety of host and genetic factors have been associated with PK variability of TB drugs such as gender, body mass index (BMI), food-drug interaction and comorbidity with HIV or diabetes as well as pharmacogenetic polymorphism most established for the influence of acetylator status on isoniazid drug concentration levels $(132,133)$.

Drug exposure can be monitored objectively and easily acted upon by the clinician unlike many other factors that may affect TB treatment outcome (e.g. treatment adherence, quality of drugs, comorbidities, TB manifestation, disease severity etc). Moreover, PK 
variability and subtherapeutic drug exposure of importance for the individual patient is difficult to predict without using TDM (42). Typically, drug concentrations of first-line drugs are measured two hours after drug intake and commonly used PK targets for therapeutic drug monitoring are the lower limit of the normal reference range; $8 \mathrm{mg} / \mathrm{L}$ for rifampicin, $3 \mathrm{mg} / \mathrm{L}$ for isoniazid, $20 \mathrm{mg} / \mathrm{L}$ for pyrazinamide and $2 \mathrm{mg} / \mathrm{L}$ for ethambutol (53). These drug concentration ranges were derived from normal distributions in healthy volunteer adults and TB patients receiving standard doses $(53,119,134)$, but they are poorly validated to clinical outcome.

There are three main purposes to use TDM in TB treatment; to reduce toxicity, to optimise drug efficacy (in terms of improved treatment outcome and reduced contagiousness) and to avoid acquired drug resistance. The latter two depend on the drug exposure in relation to the susceptibility level of $M$. tuberculosis, but only a limited number of clinical studies have correlated drug concentrations to the MIC and clinical outcome.

Several studies have identified that first-line drug levels below reference range are common during treatment for active TB, especially rifampicin and isoniazid (135). Low levels have been associated with slow response to therapy, increased risk of relapse and acquired drug resistance $(53,136)$. On the contrary, adequate treatment response despite drug concentrations below reference range for rifampicin and isoniazid have also been observed in studies (137). Table 8 presents an overview of clinical PK/PD-studies of firstline drugs including outcome measures.

A recent systematic meta-analysis did not find evidence to support routine use of TDM for drug-susceptible TB, but the studies where heterogenous in sampling strategies and few reported clinical outcome (138). There are no prospective randomized trials that clearly define the role of TDM for TB drugs. Furthermore, opinions vary regarding the utility and cost-benefit of TDM (139-141). Despite knowledge gaps, experts in the field promote the use of TDM for TB and guidelines and reviews have also acknowledged this, but not routinely for all patients $(92,108,141,142)$. Presently, therapeutic drug monitoring is mainly used in high-resource settings in case of therapy failure, MDR/XDR-TB or severe adverse drug reactions. Treatment under certain circumstances known to be prone to subtherapeutic drug levels or poor treatment outcome such as in treatment of patients with HIV co-infection, diabetes, severe TB disease, drug-drug interaction or malabsorption, is also often subjected to TDM $(140,142)$. 


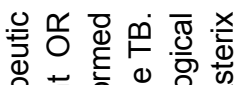

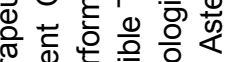
这 要

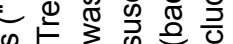
응

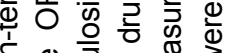

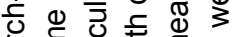
栾 ه

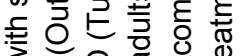
3

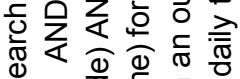

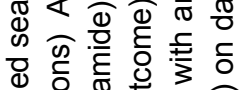

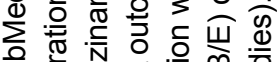

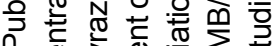

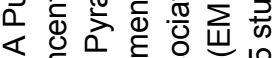
$<<0$ 당

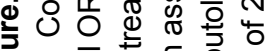

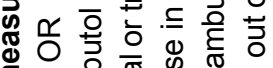

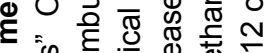

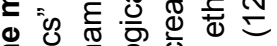

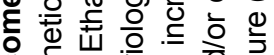

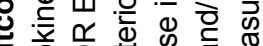
항ㅇㅎㅇㅇㅇㅇ

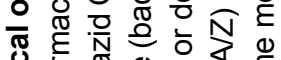
눈 등 흥해

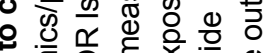
द 은 든

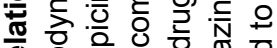

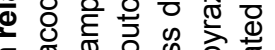

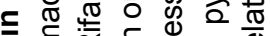

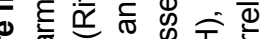

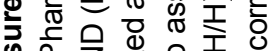

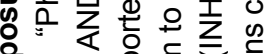

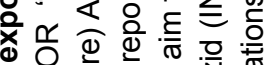

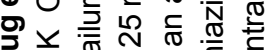

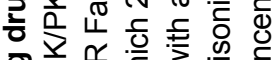

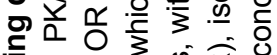

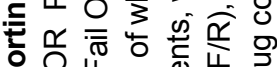

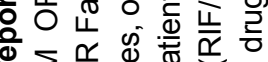

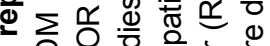
要全 严

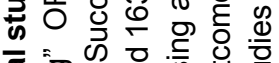
ฮ

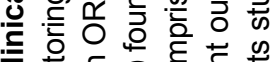

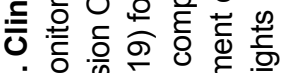

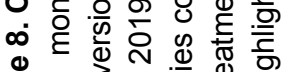

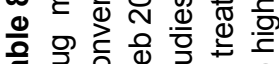

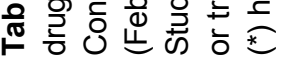

\begin{tabular}{|c|c|c|c|c|}
\hline 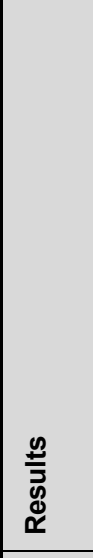 & 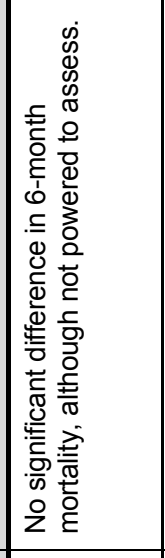 & 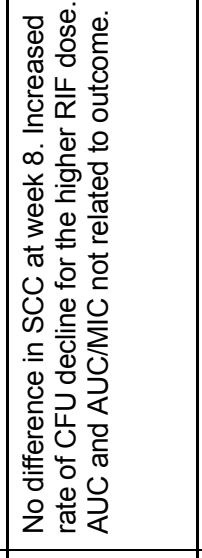 & 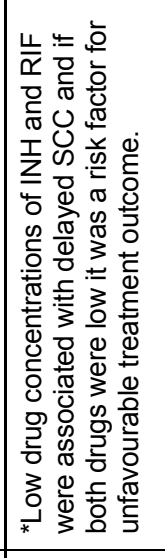 & 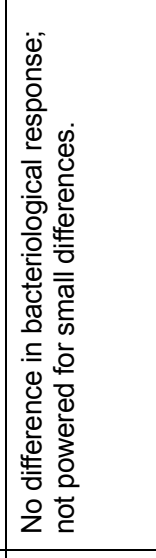 \\
\hline 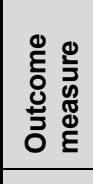 & 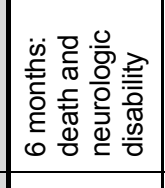 & 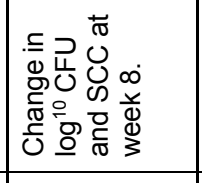 & 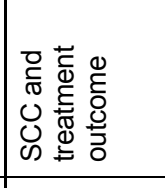 & $\begin{array}{l}0 \\
0 \\
\underline{Q} \\
\end{array}$ \\
\hline 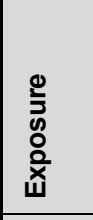 & 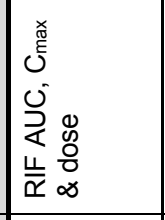 & 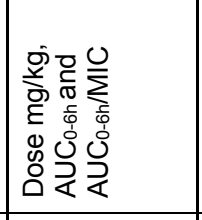 & 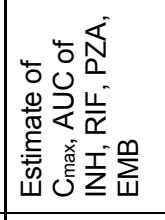 & 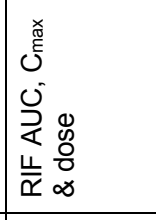 \\
\hline 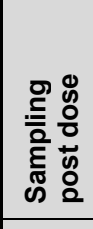 & 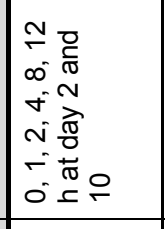 & 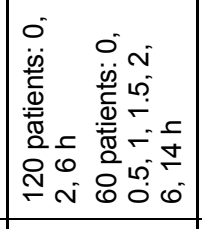 & 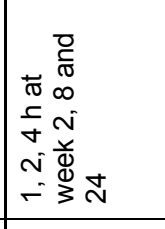 & 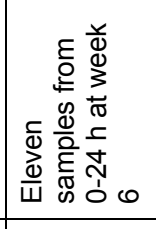 \\
\hline 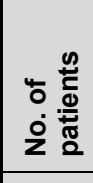 & 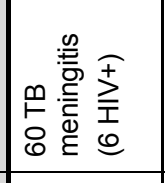 & 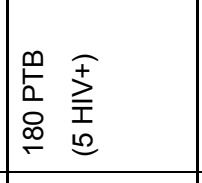 & 辤 & 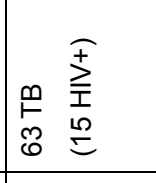 \\
\hline 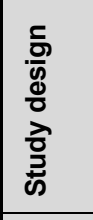 & 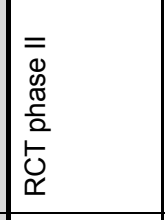 & 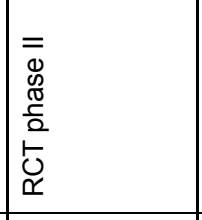 & 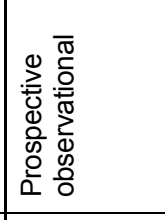 & 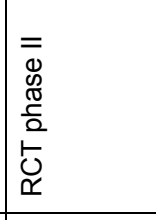 \\
\hline 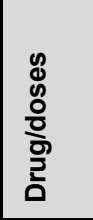 & 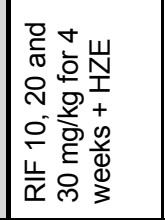 & 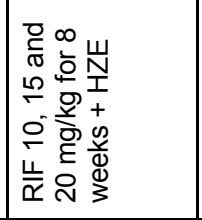 & 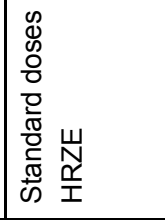 & 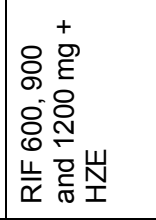 \\
\hline 辛 & 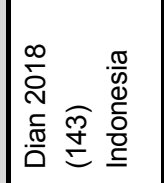 & 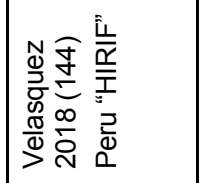 & 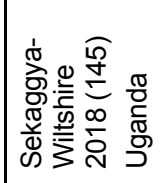 & 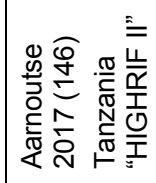 \\
\hline
\end{tabular}




\begin{tabular}{|c|c|c|c|c|c|c|}
\hline 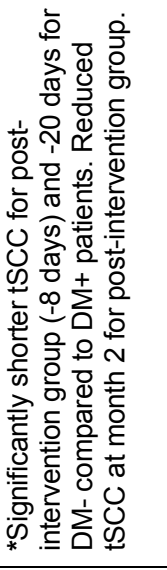 & 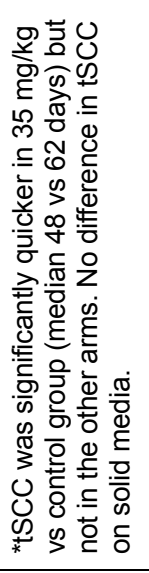 & 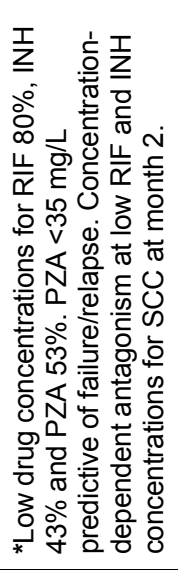 & 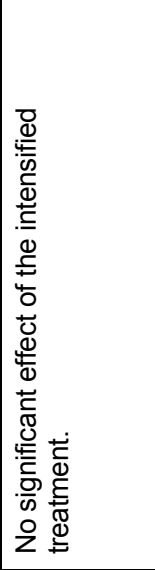 & 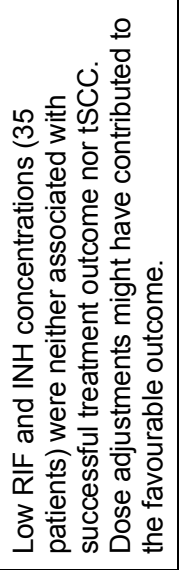 & 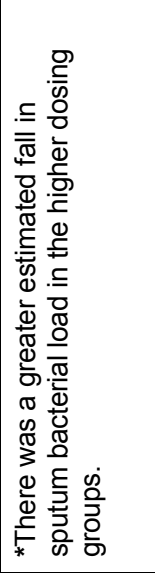 & 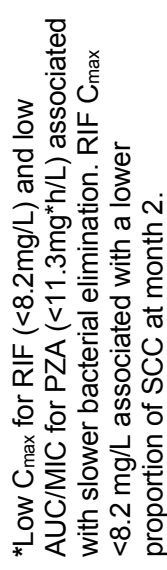 \\
\hline 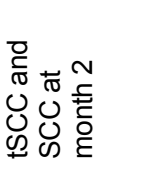 & 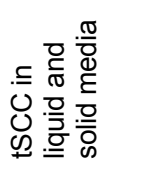 & 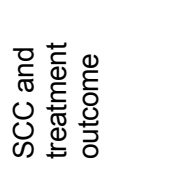 & 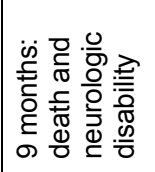 & 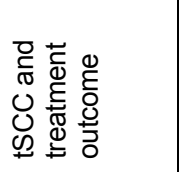 & 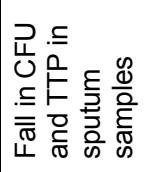 & 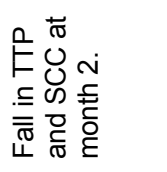 \\
\hline 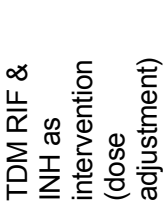 & 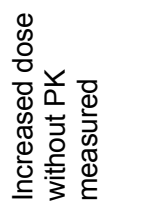 & 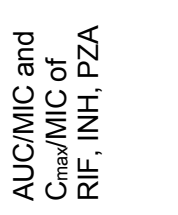 & 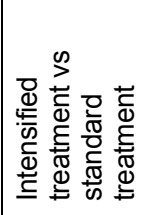 & 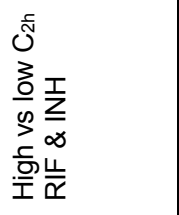 & $\begin{array}{l}\frac{u}{\bar{\alpha}} \\
\frac{1}{0} \\
\frac{x}{\alpha}\end{array}$ & 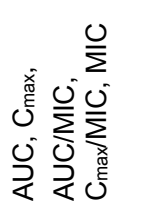 \\
\hline 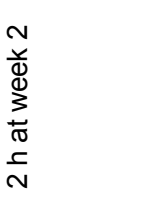 & 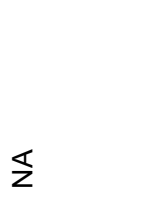 & 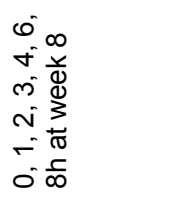 & $\S$ & $\stackrel{\check{N}}{\sim}$ & 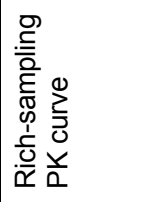 & 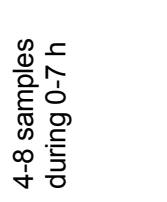 \\
\hline 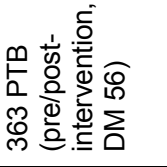 & 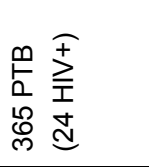 & 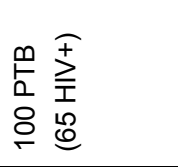 & 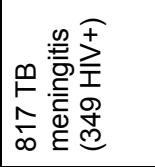 & $\begin{array}{l}\stackrel{\oplus}{\vdash} \\
\stackrel{N}{\sim}\end{array}$ & 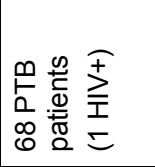 & $\begin{array}{l}\stackrel{0}{+} \\
\frac{5}{2} \\
\dot{5} \Xi\end{array}$ \\
\hline 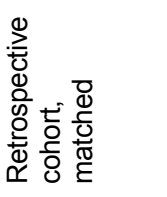 & 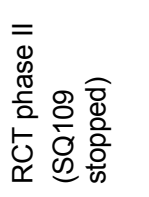 & 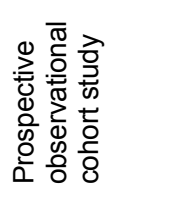 & ঢ্ & 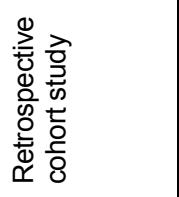 & 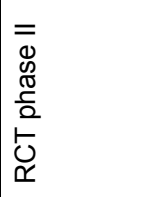 & 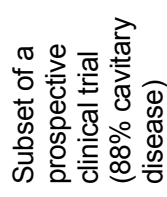 \\
\hline 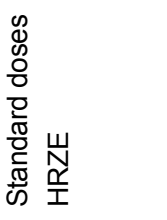 & 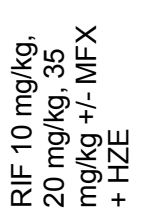 & 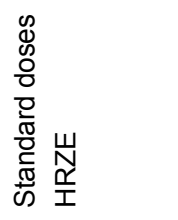 & 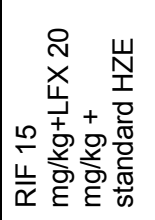 & 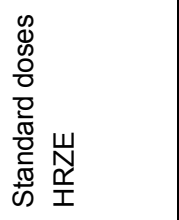 & 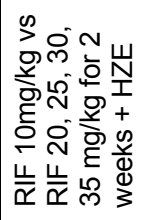 & 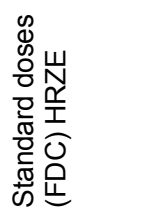 \\
\hline 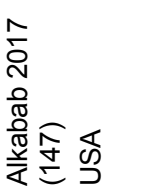 & 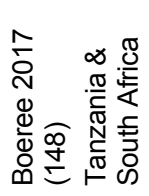 & 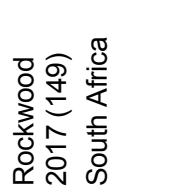 & 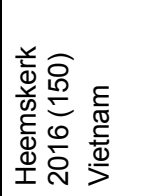 & 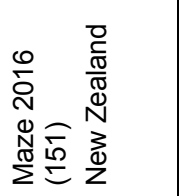 & 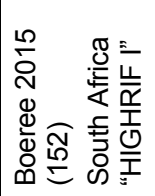 & 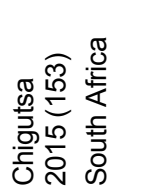 \\
\hline
\end{tabular}




\begin{tabular}{|c|c|c|c|c|c|c|}
\hline 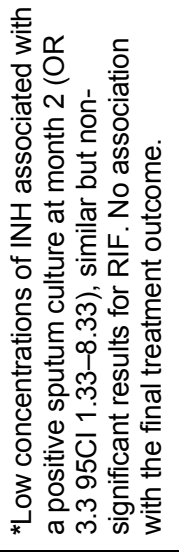 & 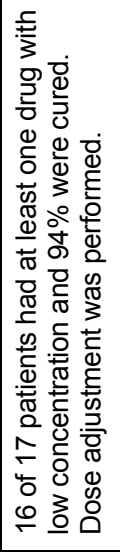 & 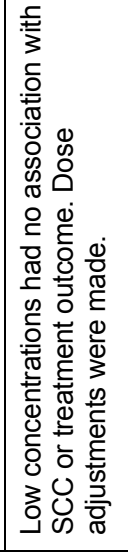 & 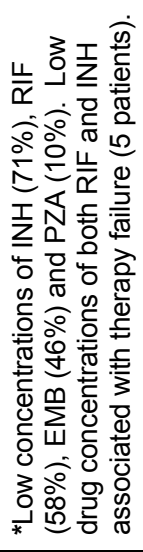 & 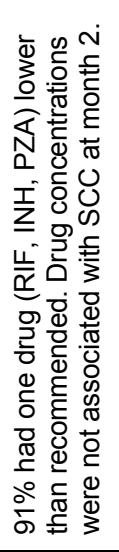 & 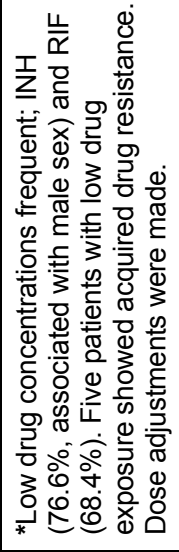 & 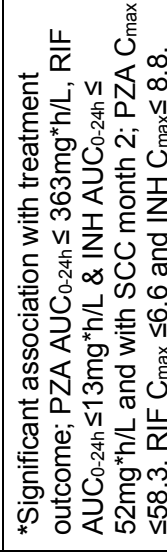 \\
\hline 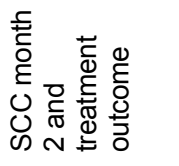 & 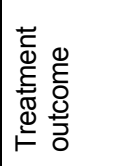 & 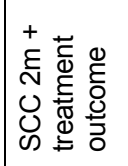 & 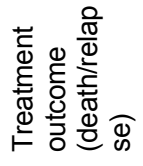 & 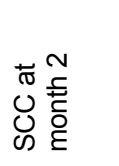 & 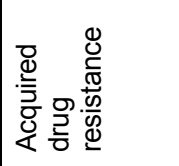 & 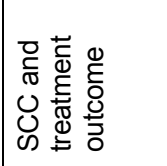 \\
\hline 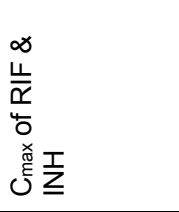 & 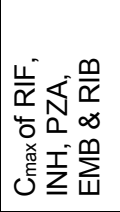 & 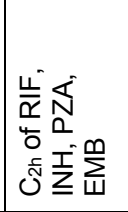 & 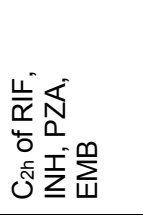 & 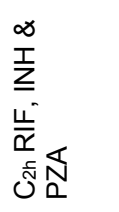 & $\begin{array}{l}\frac{I}{Z} \\
\infty \\
\infty \\
\frac{1}{\widetilde{X}} \\
\overline{\tilde{N}} \\
\mathcal{U}\end{array}$ & 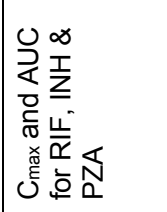 \\
\hline $\begin{array}{l}\text { ᄃ } \\
\text { N }\end{array}$ & $\begin{array}{l}0 \\
0 \\
i \\
i\end{array}$ & $\stackrel{c}{\sim}$ & $\stackrel{r}{\sim}$ & $\stackrel{\check{N}}{\sim}$ & $\stackrel{c}{\sim}$ & 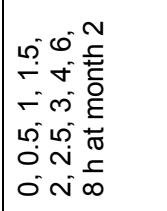 \\
\hline 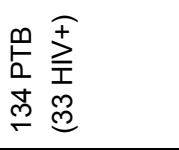 & 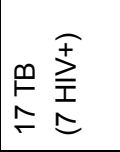 & & 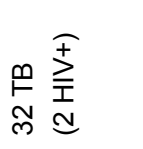 & 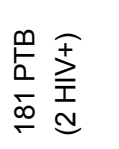 & 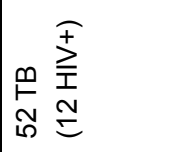 & 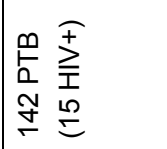 \\
\hline 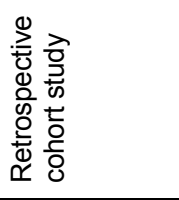 & 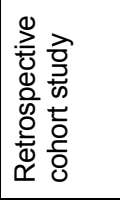 & 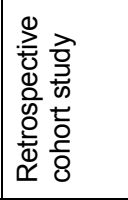 & 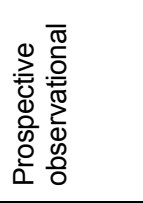 & 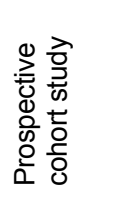 & 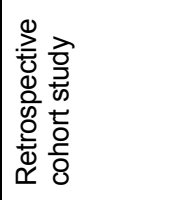 & 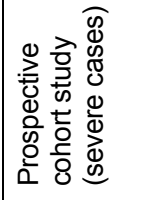 \\
\hline 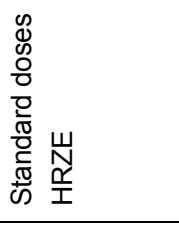 & 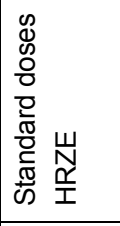 & 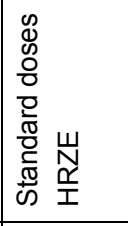 & 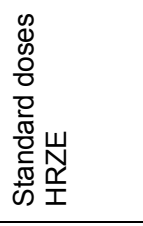 & 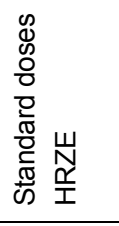 & 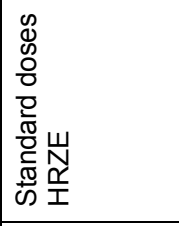 & 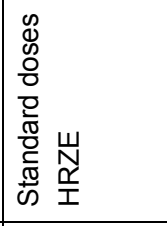 \\
\hline 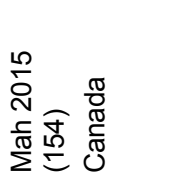 & 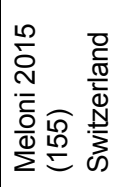 & 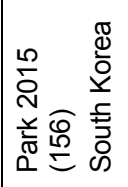 & 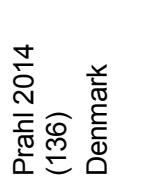 & 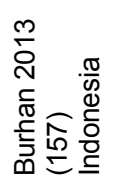 & 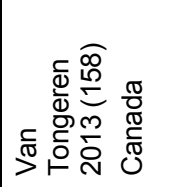 & 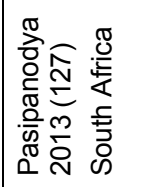 \\
\hline
\end{tabular}




\begin{tabular}{|c|c|c|c|c|c|c|}
\hline 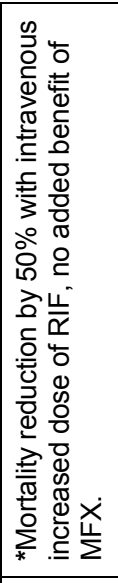 & 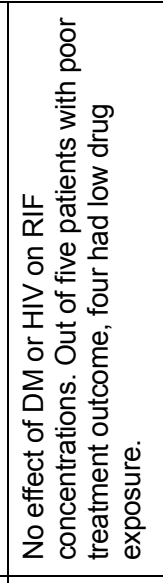 & 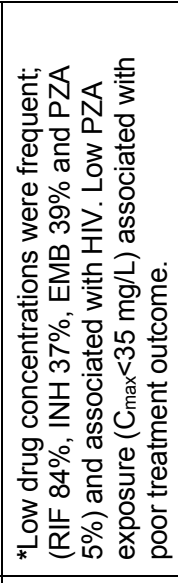 & 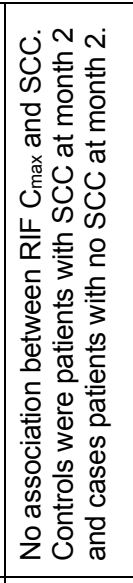 & 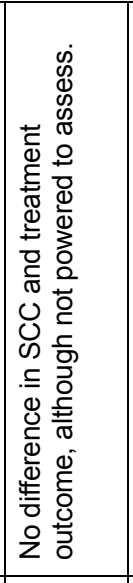 & 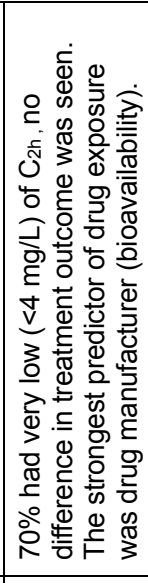 & 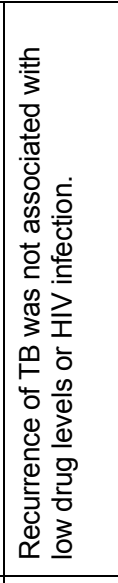 \\
\hline 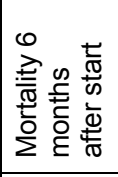 & 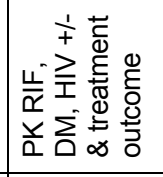 & 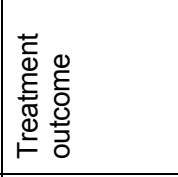 & 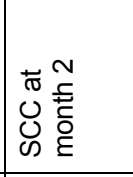 & 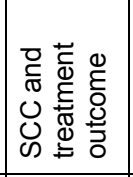 & 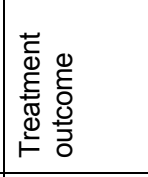 & 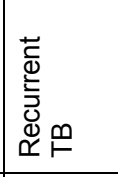 \\
\hline 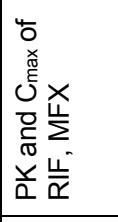 & 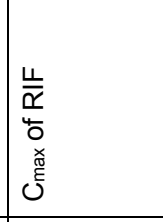 & 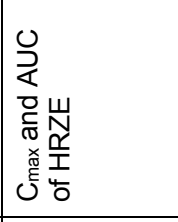 & 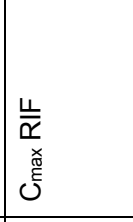 & 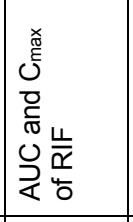 & 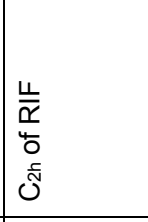 & 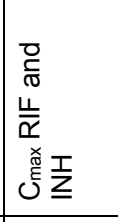 \\
\hline 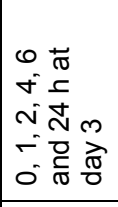 & 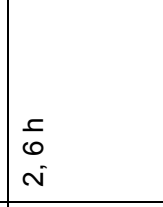 & $\begin{array}{l}c \\
\overline{6} \\
i \\
i \\
r\end{array}$ & 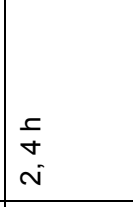 & 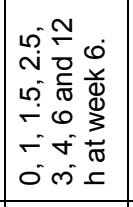 & $\stackrel{ᄃ}{N}$ & 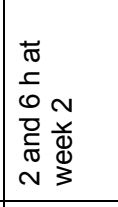 \\
\hline 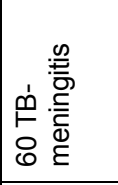 & 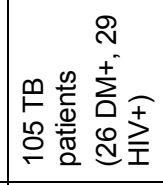 & 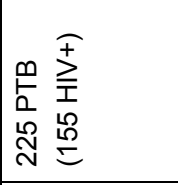 & 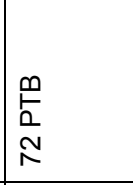 & 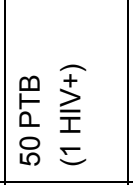 & 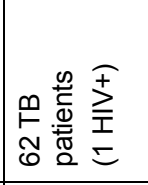 & 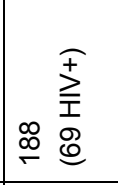 \\
\hline 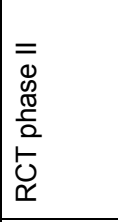 & 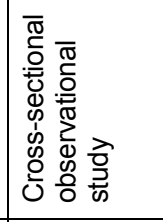 & 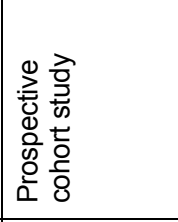 & 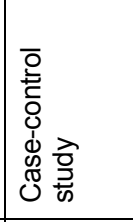 & 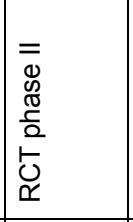 & 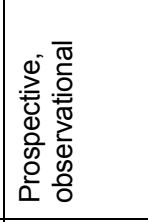 & 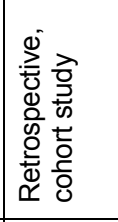 \\
\hline 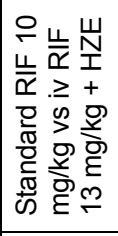 & 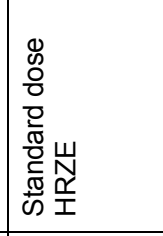 & 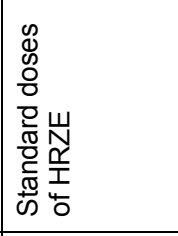 & 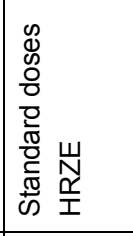 & 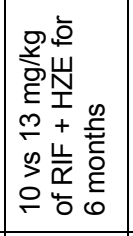 & 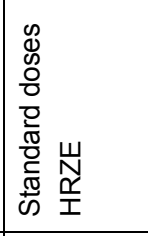 & 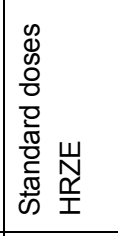 \\
\hline 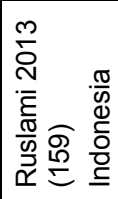 & 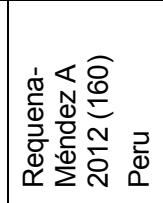 & 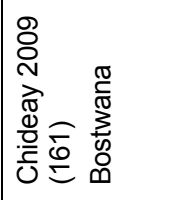 & 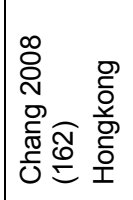 & 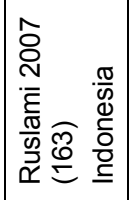 & 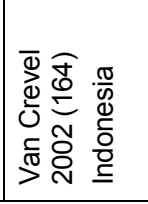 & 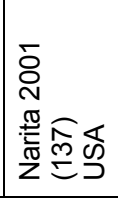 \\
\hline
\end{tabular}




\section{PK/PD relationships in TB}

Despite efforts, mainly based on animal and in vitro models, there are so far no accepted $\mathrm{PK} / \mathrm{PD}$ targets for human TB to be used for TDM purposes. Concentration-dependent bacterial killing is common for many of the TB drugs. Studies indicate the most predictive $\mathrm{PK} / \mathrm{PD}$ parameter of activity to be mainly $\mathrm{AUC} / \mathrm{MIC}$ and to some extent $\mathrm{C}_{\max } / \mathrm{MIC}$ for the first-line drugs as displayed in Table $1(13,42,51,126)$. When the fluoroquinolones were investigated in similar way the AUC/MIC was the best parameter to describe the efficacy in a murine dose fractionation study (64). However, an important reminder is that the studies providing these data are mainly based on monotherapy in murine models. Furthermore, the MIC determination method has an inherent technical variability and different methods for MIC determination were used.

One study in the hollow fibre system model suggested a ratio of free rifampicin $\mathrm{C}_{\max } / \mathrm{MIC}$ $\geq 175$ to attain optimal bactericidal effect and prevent acquired drug resistance (165). In a murine aerosol infection model based on dose fractionation of rifampicin over six days, the $\mathrm{AUC}_{0-24 \mathrm{~h}} / \mathrm{MIC}$ ratio needed for $1-\log _{10} \mathrm{CFU}$ reduction of the total lung bacterial load was 271 and the killing activity was also correlated to $\mathrm{C}_{\max } / \mathrm{MIC}$ but not $\% \mathrm{~T}_{>\mathrm{MIC}}(51)$. This PK/PD-ratio was based on monotherapy and human PK-population studies coupled with Monte Carlo simulations have reported low probabilities of target attainment at rifampicin $600 \mathrm{mg}$ daily $(166,167)$. Both the hollow fibre system and mice model have reported that an isoniazid $\mathrm{AUC}_{0-24 \mathrm{~h}} / \mathrm{MIC}>60$ was needed to achieve a $50 \%$ microbial kill effect $(126,168)$. The pyrazinamide PK/PD parameter linked to sterilizing effect in the hollow fibre system model has been suggested to be AUC/MIC, with an optimal value of about 210 at site of effect and Monte Carlo simulations indicate that the current standard dose of pyrazinamide is suboptimal (13). In one of the few clinical studies able to derive tentative PK/PD-targets (although without MIC determinations) for first-line drugs included 142 drug-susceptible pulmonary TB patients whereof $25 \%$ experienced poor long-term outcomes (127). Thresholds predictive of this outcome were a 24-hour AUC of $363 \mathrm{mg} \cdot \mathrm{h} / \mathrm{L}$ for pyrazinamide, $13 \mathrm{mg} \cdot \mathrm{h} / \mathrm{L}$ for rifampin, and $52 \mathrm{mg} \cdot \mathrm{h} / \mathrm{L}$ for isoniazid. Moreover, in this study low rifampicin and isoniazid peak and AUC concentrations preceded the three cases of acquired drug resistance. Less is known about PK/PD relationship for second-line drugs, but hollow fibre studies of moxifloxacin, gatifloxacin and levofloxacin against $M$. tuberculosis have suggested free $\mathrm{AUC}_{0-24 \mathrm{~h}} / \mathrm{MIC}$ of 53, 184 and 360 , respectively to completely supress drug-resistant mutant population. Similar ratios were identified to be associated with $80 \%$ of maximal microbial kill effect except for levofloxacin $\left(\mathrm{AUC}_{0-24 \mathrm{~h}} / \mathrm{MIC}\right.$ of 146$)(65,169,170)$. 
"The world is full of obvious things which nobody by any chance ever observes." Sir Arthur Conan Doyle 



\section{AIMS}

\section{Overall aim of the thesis}

The overall aim of this thesis was to investigate the current fluoroquinolone susceptibility testing breakpoints for $M$. tuberculosis, the pharmacokinetics and pharmacodynamics of TB treatment and to explore the potential role of therapeutic drug monitoring for optimising TB treatment.

\section{Specific objectives of the included studies}

- To investigate how discrepancies in the phenotypic and genotypic methods for drug susceptibility testing of fluoroquinolones could affect the interpretation of drug susceptibility test results for M. tuberculosis. (Study I)

- To explore plasma concentrations of first-line TB drugs in a cohort of TB patients in a low-endemic setting, along with corresponding individual MICs. (Study II)

- To investigate whether the TB drug activity assay (TDA) could be used as an indicator of plasma concentrations of rifampicin. (Study III)

- To develope a study protocol with the aim to investigate the drug exposure of second-line drugs and corresponding individual MICs, in relation to markers of clinical outcome in a cohort of MDR-TB patients in China. (Study IV) 



\section{METHODOLOGICAL CONSIDERATIONS}

In this part of the thesis, the main methods used in the different studies are discussed in terms of choice of methods and their strengths and limitations. More detailed description of the specific methods can be found in the original articles.

\section{Methods of phenotypic DST and determination of MIC}

The strength of using culture-based DST for M. tuberculosis is that it, in theory, detects all resistance regardless of the mechanism (171). By adding determination of MIC it is also possible to perform a quantification of the susceptibility level. Major downsides of phenotypic tests are that they are complex, labour-intense and have long turnaround time, around 3-6 weeks depending on the time needed for the primary culture to turn positive. The lack of a universal standardised reference method for pDST leads to intra- and interlaboratory variabilities due to potential differences in preparation of stock solutions and bacterial inoculum, which in turn affects the accuracy and reproducibility of the test. Other possible sources of error include procedure-related loss of incorporated drug, media $\mathrm{pH}$, temperature and incubation time (77). Reliable MICs are needed for studies evaluating potential $\mathrm{PK} / \mathrm{PD}$ targets and several different methods are unfortunately in use without any reference method. To facilitate interpretation of pDST and MIC results between studies it is pivotal to include internal controls with a quality control strain such as M. tuberculosis H37Rv ATCC 27294 (172). In general, there is good agreement in pDST results between the available assays based on the proportion method (e.g. Löwenstein-Jensen, 7H10, BACTEC MGIT) when compared head-to-head $(173,174)$. Reproducibility is an issue with susceptibility testing of pyrazinamide, as it is technically difficult because of the acid $\mathrm{pH}$ requirement for drug activity (175). Liquid broth systems with low $\mathrm{pH}$ medium must be used and many clinical microbiology laboratories do not perform pDST of pyrazinamide. Low $\mathrm{pH}$ itself inhibits growth of $M$. tuberculosis isolates why a bacterial suspension of 1:10 instead of 1:100 is used as a growth control (176). False pyrazinamide resistance is by far the most common problem as $\mathrm{pH}$ increases if the inoculum size is too large (175).

MIC determination was performed in study I by using solid Middlebrook 7H10.

A large number of MIC determinations were performed in study $\mathbf{I}$ in order to elucidate the correlation between phenotypically wild-type and non-wild-type strains for fluoroquinolones in relation to currently used critical concentrations and the presence of mutational resistance. Since the indirect proportion method is the most commonly used strategy for pDST of M. tuberculosis this was preferred to other techniques. MIC determination using the proportion method on Löwenstein-Jensen or in BACTEC MGIT 960 requires large amounts of media and is time-consuming. Hence, the modified indirect proportion method on Middlebrook 7H10 agar plates combined with a 96-stick replicator for transfer of bacterial inoculums was chosen as this method enables simultaneous testing of large number of isolates. The MIC was defined as the lowest drug concentration with less visual growth than the 1:100 diluted control of the corresponding strain, thereby 
representing inhibition of $>99 \%$ of the bacterial population at the MIC. Even if the manual visual reading of plates in $7 \mathrm{H} 10$ can be difficult, good concordance has been noted between 7H10 and the automated liquid system BACTEC MGIT 960 (173). Since there is no standardised reference method for MIC determination and technical variability is a concern, M. tuberculosis H37Rv ATCC 27294 was included in duplicates in each batch of MIC testing as an internal quality control of the inter-assay variability. A strength in study I is the stable results of the quality control strain with MIC-variability within \pm 1 two-fold dilution step, indicating a robust test system. Quality control isolates are rarely included in MIC determinations of M. tuberculosis impeding the comparison of MIC results between different laboratories as noted in a recent systematic review (59).

In study II, MIC was determined in BACTEC MGIT 960 and in liquid broth microdilution, the latter method also used in study IV.

In Sweden BACTEC MGIT 960 is the routine method for pDST of TB drugs and the only available routine method for pDST of pyrazinamide, therefore this method was used for MIC determinations in study II. The MIC was defined as the lowest antibiotic concentration where there was less growth compared with the 1:100 diluted control (for pyrazinamide 1:10 diluted control). The MIC results retrieved from MGIT were compared with results from Sensititre ${ }^{\mathrm{TM}}$ MYCOTB MIC plates (except for pyrazinamide) and the two methods were in agreement within \pm 1 two-fold MIC dilution step in $>95 \%$ of the observations. Albeit, broth microdilution is not yet a standard method and the sensitivity of detecting drug resistance compared with BACTEC MGIT 960 is still variable (e.g. $>95 \%$ for rifampicin, $72 \%$ for ethambutol) (85). Moreover, the prefabricated MIC-dilution steps are not always adjusted to include the full MIC wildtype distribution leading to truncations of MIC results. To further substantiate the MIC results in study II, the M. tuberculosis isolates were screened for high-confidence and established resistance mutations for first-line drugs by WGS. All isolates were genotypic wild-type including the few isolates with elevated MIC values below but close to the critical concentrations. M. tuberculosis H37Rv ATCC 27294 was included in duplicates in each batch of MIC testing as an internal quality control of the inter-assay variability. For pyrazinamide and isoniazid there was no measurable variability whereas rifampicin and ethambutol showed an inter-assay variability of \pm 1 two-fold MIC dilution step. In study IV MIC was determined in custom-made TREK Sensititre ${ }^{\mathrm{TM}}$ broth microdilution method as it is a simple, cost-effective and high-throughput method for large number of simultaneous MIC determinations in several drugs. The MIC was defined as the lowest antibiotic concentration where there was less visual growth compared with the 1:100 diluted control read after 10-21 days, depending on growth, assisted by an inverted mirror.

\section{Methods of genotypic DST}

Sanger sequencing of the quinolone resistance-determining region (QRDR) of gyrA was used in study I for genotypic testing as well as the Genotype ${ }^{\circledR}$ MTBDRsl assay version 1.0. Whole genome sequencing was used in study II and IV. 
Genotypic DST has the advantages of being able to deliver rapid results within hours or days and some of the techniques do not require bio-safety laboratory infrastructure (e.g. Cepheid GeneXpert ${ }^{\circledR}$ System). The exact correlation between genotype and phenotype for some mutations remains unclear, which complicates the interpretation of the assays, especially for WGS with rich out-put data (101). A common feature of DNA-based assays is that they only screen the nucleic acid sequence, and therefore it is possible that detected mutations do not cause an amino acid exchange. Problems with systematic false-resistant results has been highlighted as for MTBDRsl with synonymous and nonsynonymous mutations including their influence on the binding of mutant probes and wild-type bands $(177,178)$.

\section{Pharmacokinetic analysis}

Pharmacokinetic parameters used in the different studies were; $\mathrm{C}_{2 \mathrm{~h}}, \mathrm{C}_{\mathrm{high}}, \mathrm{AUC}_{0-6 \mathrm{~h}}$ in study II, $\mathrm{C}_{2 \mathrm{~h}}$ in study III and $\mathrm{C}_{\max }$, AUC in study IV.

Calculation of $\mathrm{AUC}_{0-24 \mathrm{~h}}$ requires rich sampling for drug concentration measurements (often approximately 10 observations). Rich sampling is also needed for calculation of $\mathrm{C}_{\max }$ as delayed drug absorption may occur which leads to increased time to $\mathrm{C}_{\max }\left(\mathrm{T}_{\max }\right)$. Calculation of $\mathrm{AUC}_{0-t}$ by linear trapezoidal method as delineated in Figure 7 applies well for drugs with linear kinetics and for the increasing and equal concentrations (the plateau), meanwhile the log trapezoidal method preferably is used for calculation of the exponential part of the declining curve $(120,122)$.

As sparse sampling over the first 6 hours without access to PK modelling was used in study II, an AUC that covered a shorter time frame of the total AUC was calculated by using the linear trapezoidal method. Even if this reflected the main part of the total AUC for rifampicin and isoniazid as shown in Figure 14, $\mathrm{AUC}_{0-6 \mathrm{~h}}$ is an underestimate and crude measurement of the total AUC. The sparse sampling in study II also precluded assessment of the individual $\mathrm{C}_{\max }$ therefore the highest received drug concentration at 0 , 2, 4 and 6 hours was used and denoted $C_{\text {high. }}$. In study IV a richer sampling strategy was applied. The relationship between dose, concentration and effect will be assessed by using PK modelling and the nonlinear mixed-effects models will be applied.

\section{Ethical considerations}

In study II in Sweden, all patients received oral and written information regarding the research project and provided written informed consent. The study was approved by the regional ethics committee (DNR 2012/197-31) and was conducted in accordance with the principals of the Declaration of Helsinki. Study IV in China has just been closed for recruitment and prior to study opening, a designated study team participated in training workshops of the study protocol and ethical considerations. This was led by the main study investigators from Fudan University, Shanghai and Karolinska Institutet, Stockholm with the purpose to assure study compliance with Good Clinical Practice and the Declaration of Helsinki. Prior to study inclusion patients were informed (orally and in writing) about the study and signed (in case of illiteracy witnessed fingerprint was used) written informed consent. Ethical approval was obtained from the Ethical Review 
Board of Stockholm (EPN: 2015/646 31/1) and the Institutional Review Board of the School of Public Health, Fudan University, China (IRB 2015-09-0565).

Extensive blood sampling is a sensitive issue in some cultures (especially in China but also in parts of Africa) therefore the number of blood samples and amount of blood in both Study II and IV have been reduced to a minimum for estimation of peak drug concentration and AUC. Increased sputum sample collection is a potential hazardous risk for other patients, hospital and laboratory staff. Precautions have been taken with instructions for sputum sample collection and in study IV in China an upgrade of the biosafety equipment has been implemented. 


\section{MAIN RESULTS AND DISCUSSION}

\section{General discussion}

Tuberculosis is a multi-faceted challenge to both patients, clinicians and health care systems.

Although often located to the lungs, the disease is quite the opposite from the often easyto-treat community acquired pneumonia. As being one of the "big imitators" with often slowly progressing disease which initially is subclinical, patient's and doctor's delay is a reality which fuels further spread of the disease. Tuberculosis treatment is difficult and complex with the mandatory inclusion of several drugs for a long time period due to different metabolically active subpopulations of bacilli. This holds the risk of poor treatment adherence and emergence of acquired drug resistance. As if these facets were not enough there are several short-comings in diagnostics, DST and breakpoints, PK/PD targets and biomarkers for treatment evaluation - the TB field is far behind achievements accomplished for other bacterial pathogens. On top of this, the largest hazard is the worrisome increase in the man-made phenomenon MDR/XDR-TB.

In terms of treatment of TB, the use of repurposed drugs and development of new TB drugs are not the only solutions to combat $\mathrm{TB}$, as resistance already has appeared against bedaquline and delaminid (5). This oblige all clinicians and health care workers to put effort in optimising the current treatment regimens. To be able to give the most effective treatment to every patient and to find stratified treatment regimens for certain groups of TB patients, it is elementary to have well defined cut-offs between drug susceptible and drug resistant TB. As a clinician you need to know if your TB patient has a fully drugsusceptible $M$. tuberculosis isolate, dose-dependent low-level of resistance within reach of treatment or evidence of high-level resistance that requires exclusion of one or more drugs from the treatment. Moreover, it is important when designing a drug regimen for TB to consider the complicated relationship between host (immune defence, comorbidities, pharmacogenetics), pathogen (virulence, site of infection, disease severity) and drug exposure (PK properties of the drug).

Measuring plasma drug concentrations along with $M$. tuberculosis susceptibility level in relation to treatment outcome can shed some light towards finding relevant PK/PD targets for TB drugs. Targets of importance for treatment guidance in personalised medicine based on therapeutic drug monitoring as well as being useful in the design of new shorter treatment regimens against drug-susceptible and drug-resistant TB. 


\section{The importance of reliable drug susceptibility testing}

\section{Imperfect correlation between current breakpoints and resistance mutations}

In study I, the main finding was that levofloxacin and ofloxacin-resistant $M$. tuberculosis strains, defined by the presence of specific codon 90 mutations in the quinolone resistance-determining region (QRDR) of gyrA, had MIC levels in close relationship to the wild-type organisms. In 2014, the WHO revised the critical concentrations from 2 $\mathrm{mg} / \mathrm{L}$ to $1 \mathrm{mg} / \mathrm{L}$ for levofloxacin on Middlebrook $7 \mathrm{H} 10$, and for ofloxacin it was maintained at $2 \mathrm{mg} / \mathrm{L}$ (21). These breakpoints were recently reaffirmed (Table 5) by the WHO, based on a technical report with the purpose to assess evidence for the different critical concentrations used in pDST of second-line drugs (59). Current breakpoints on Middlebrook $7 \mathrm{H} 10$ may classify up to $25 \%$ of $M$. tuberculosis isolates with resistance mutations in gyrA (mainly in codon 90) as susceptible to fluoroquinolones (Figure 10 and 11). Resistance mutations in codon 90 (most notably A90V) were associated with MICs close to the MICs of the wild-type organisms indicating low-level resistance, in contrast to most mutations in codon 94 exhibiting higher MIC levels as displayed in Figure 10 and 11. The phenotypic difference in fluoroquinolone susceptibility between strains with certain mutations in codon 90 versus 94 has been observed in other studies including for fluoroquinolones not covered in study I; gatifloxacin and moxifloxacin $(59,179,180)$. This is useful information for the treating clinician (if obtainable from rapid genotypic testing) as strains with mutations conferring low-level resistance (such as in codon 90) may be treatable with increased drug exposure, which rarely is the case for mutations conferring high-level resistance. 


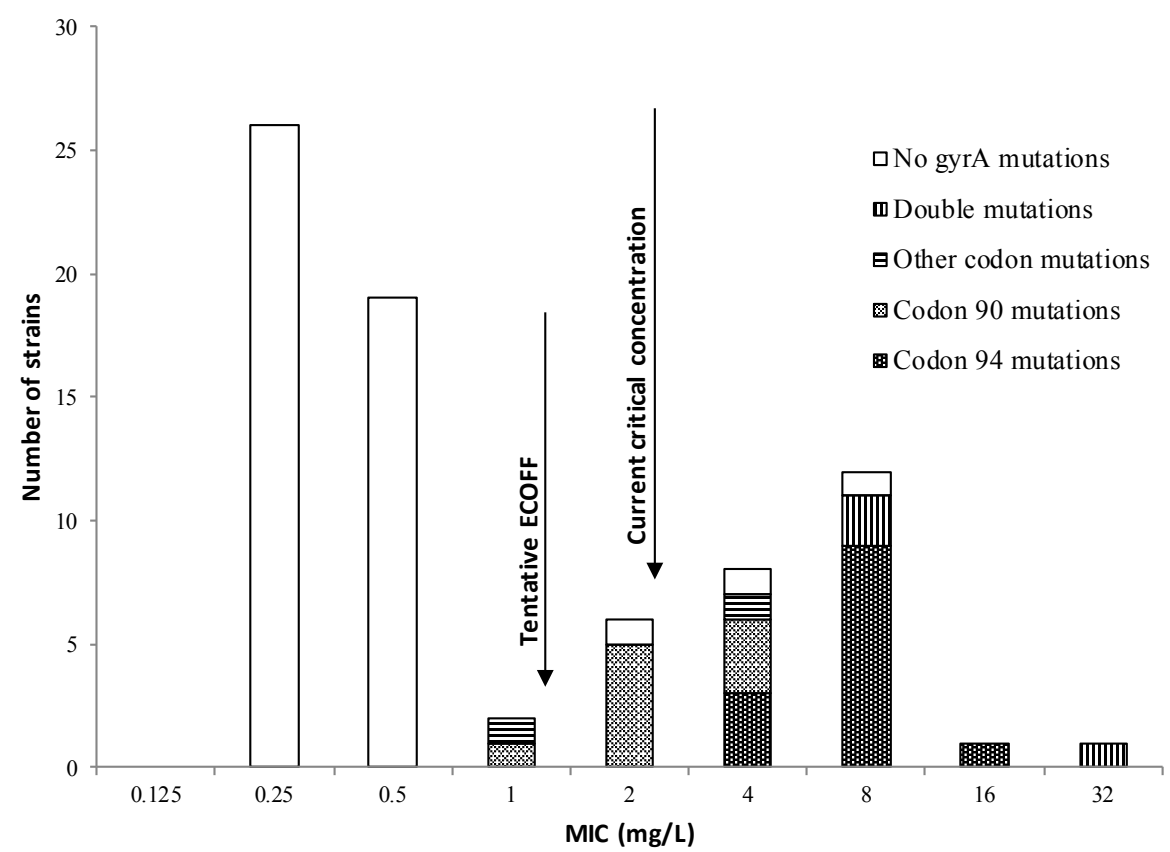

Figure 10. MIC distribution in 7H10 of ofloxacin in relation to mutations in gyrA. Seven isolates with mutations in gyrA are below the current breakpoint (critical concentration) at $2 \mathrm{mg} / \mathrm{L}$. 


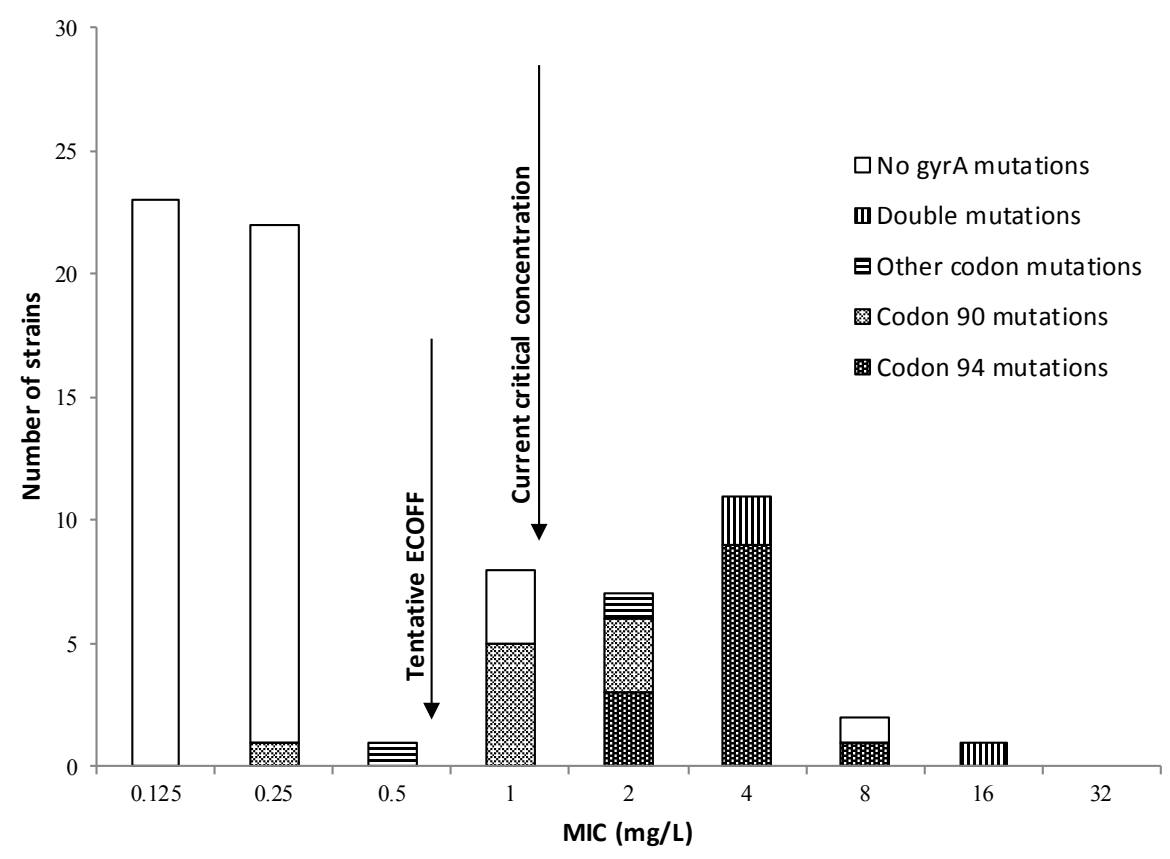

Figure 11. MIC distribution in 7H10 of levofloxacin in relation to mutations in gyrA. Seven isolates with mutations in gyrA are below the current breakpoint (critical concentration) at $1 \mathrm{mg} / \mathrm{L}$.

In line with the main finding in study $\mathbf{I}$, it was repeatedly noted in the aforementioned technical report by the WHO that due to the methodological variability of pDST for $M$. tuberculosis certain gyrA mutations "spanned the CC" (i.e. exhibited MICs close to the critical concentration) and can be misclassified as susceptible by the currently used critical concentrations for ofloxacin and levofloxacin (59). Inclusion of moxifloxacin in study I would have allowed for analysis of cross-resistance among fluoroquinolones. Nevertheless, the same issue of potential misclassification of non-wild-type strains as phenotypically susceptible to moxifloxacin was observed in the technical report. Sufficient genotype-phenotype data were available for moxifloxacin to identify a lowlevel (mainly A90V) and high-level resistance (mainly D94G). The WHO therefore revised the current breakpoints for pDST of moxifloxacin in order to avoid underreporting of drug resistance and to communicate the possibility of using higher drug dosages for treatment of strains with low-level resistance (59). Although fluoroquinolone resistance is mostly linked to mutations in the gyrA gene (64\%; 786 of 1220 isolates) and to a lesser extent in the $g y r B$ gene $(5.6 \% ; 30$ of 534 isolates) according to a systematic review (181), additional sequencing of gyrB in study I may have uncovered some additional discrepancies between current breakpoints and resistance mutations.

The stability of the internal quality control strain $(\mathrm{H} 37 \mathrm{Rv})$ included in the MIC determinations in study I in combination with similar findings in the technical report, substantiate that current critical concentrations for ofloxacin and levofloxacin split the 
MIC-distribution of the resistant population harbouring mutations conferring low-level fluoroquinolone resistance.

The rifampicin wild-type distribution of $M$. tuberculosis is in general better separated from the resistant population compared with what has been observed for e.g. the fluoroquinolones, and the currently used critical concentrations work well in clinical practice $(182,183)$. However, there are reports on rare cases of phenotypically rifampicin susceptible isolates according to current breakpoints, carrying specific mutations in rpo $B$ $(184,185)$. If such isolates will respond to standard doses of rifampicin remains to be shown in clinical trials.

Ethambutol MIC distribution of wild-type and resistant isolates exhibit a close relationship in a similar way as for fluoroquinolones, and the current breakpoint of $5 \mathrm{mg} / \mathrm{L}$ in BACTEC MGIT 960 and the tentative ECOFF of $4 \mathrm{mg} / \mathrm{L}$ are close to the MICs of isolates with resistance-conferring mutations in $\operatorname{embB}(101,103)$. Isolates with disagreement between $7 \mathrm{H} 10$ and BACTEC 460 has been observed to surround the ethambutol breakpoint (MIC 4-8 $\mathrm{mg} / \mathrm{L}$ ) illustrating the random effect on pDST results due to the inherent technical variability (103).

The poor correlation between some of the critical concentrations (i.e. fluoroquinolones and ethambutol) set for TB drugs and resistance mutations illustrates the difficulties in defining single binary breakpoints, without considering the wild-type distribution and the continuous resistance in $M$. tuberculosis which preferably should be classified as lowand high-level resistance.

\section{Consequences of poorly defined breakpoints}

Considering the methodological variation of up to \pm 1 two-fold dilution step for MIC determination, it is likely that if there is a close relationship between the wild-type and non-wild-type distribution, as is the case with the fluoroquinolones with breakpoints set near the ECOFF, some strains with resistance mechanisms conferring a moderate increase in MIC, could have susceptibility test results that oscillate between S and R upon repeated testing according to current critical concentrations. This has implications for the classification of XDR-TB and for patients treated for MDR-TB which may be at risk of developing further drug resistance and therapy failure. It would have been interesting to investigate the treatment regimens and clinical outcome data connected to the different strains used in study I even if such studies are difficult to interpret due to variability in disease severity and background drug regimens.

Although breakpoints for TB drugs set by the WHO are fundamental to all treatment decisions in clinical practice and equally important in TB research, the evidence is accumulating that for key TB drugs the current breakpoints defining "susceptibility" and "resistance" are not suitable to distinguish between low- and high-level of drug resistance $(103,186)$. Poorly reproducible pDST results may introduce bias in clinical studies evaluating treatment outcome due to the risk of arriving at false conclusions. The initiated effort and work by several stakeholders like the WHO and EUCAST, to improve the accuracy of pDST breakpoints for $M$. tuberculosis is important for treatment management. Until a universal reference method for pDST has been established and breakpoints properly revised, it is advisable in difficult cases with complicated resistance 
to use pDST in combination with gDST because of the influence of the inherent technical variability of pDST. Further, a reliable standardised pDST is needed to draw the map of genotype-phenotype correlations by gathering data on the phenotypic profiles of the different drug resistance mutations, as well as for resistance screening of new drugs entering the market (92). Hopefully in the future, WGS will have the ability to relate all resistance mutations of TB drugs to phenotypic susceptibility, enabling early design of an adequate treatment regimen and correct dose prescriptions. Excellent work has been initiated by the Cryptic Consortium for the first-line drugs, although their analysis of genotype-phenotype did not include correlations to clinical outcome (79).

\section{Is re-evaluation of breakpoints for M. tuberculosis according to the SIR- system warranted?}

One solution to circumvent the misclassifications of TB drugs with a close relationship between wild-type and resistant populations, could be to apply an "area of technical uncertainty" (ATU) as used in the combined interpretation of wild-type/non-wild-type MIC distribution, ECOFF and tentative breakpoints for other bacterial pathogens (www.eucast.org).

The suggested MIC in Middlebrook 7H10 representing an ATU for levofloxacin would be $1 \mathrm{mg} / \mathrm{L}$ (Figure 11) based on the results for levofloxacin in Study I and gathered MIC distribution data of wild-type and non-wild-type including resistance-conferring mutations $(59,187)$. However, it needs to be substantiated by more data from several laboratories. Within an ATU the laboratory is advised to take actions to unravel technical difficulties and discern resistance primarily by a gDST (e.g. WGS or MTBDRsl V2.0 for detection of high-spot mutations in $g y r A$ and $g y r B$ ) or by repeated pDST if a technical error is suspected. Whether isolates with slightly elevated levofloxacin MIC above the ECOFF, but below current breakpoint and confirmed to be carrying resistance mutations such as in codon 90, should be communicated to the clinician as "susceptible, increased exposure" (Figure 12) or low-level resistant, depends on available PK/PD and/or clinical outcome data in support of using higher doses $(92,123)$. 


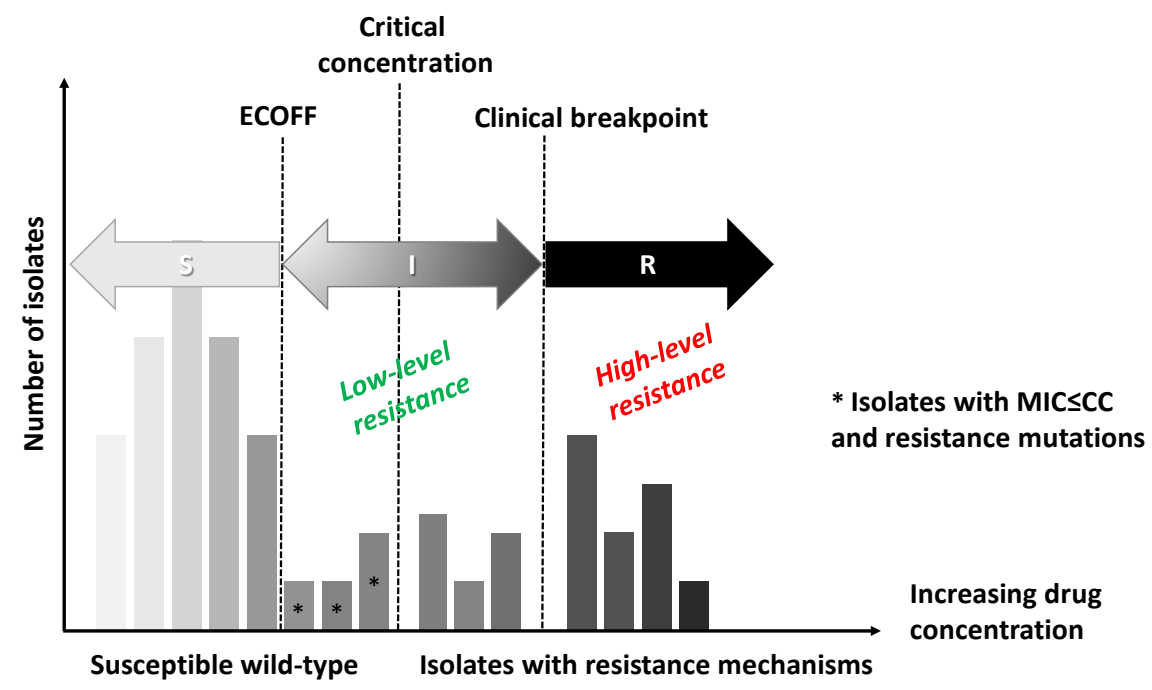

Figure 12. Schematic illustration of SIR-classification applied to M. tuberculosis. Isolates with increased drug resistance compared to ECOFF and/or the critical concentration with high likelihood of therapeutic success if the drug dose is increased, belonging to an intermediate category, or "susceptible, increased exposure" (I). The number of MIC dilution steps has been increased for clarity.

Alternatively, there could be evidence for achieving adequate drug exposure in relation to MIC by using standard drug doses, for instance with the highest recommended standard dose followed by TDM to ensure recommended drug level. Standard doses of levofloxacin according to the WHO guidelines are $10-15 \mathrm{mg} / \mathrm{kg}$, with an actual dose range of 750-1000 mg once daily (21). Clinical studies have indicated difficulties to reach suggested PK/PD targets (expert opinion based $f \mathrm{AUC}_{0-24 \mathrm{~h}} / \mathrm{MIC}$ ratio $>100 ;(64,188)$ ) on standard doses of levofloxacin as part of a MDR/XDR-TB treatment, in particular if MIC is close to the critical concentration (189). Recently, a hollow fibre study suggested a levofloxacin $f \mathrm{AUC}_{0-24 \mathrm{~h}} / \mathrm{MIC}$ of 146 to be associated with $80 \%$ of maximal $M$. tuberculosis kill and 360 for suppression of resistance (65). TDM has been advocated to ensure adequate drug exposure if MIC is $\geq 0.5 \mathrm{mg} / \mathrm{L}$ (190). Good target attainment for simulated MICs from 0.25 to $0.5 \mathrm{mg} / \mathrm{L}$, has been shown in a clinical study on levofloxacin pharmacokinetics, in patients with MDR/XDR-TB combined with Monte Carlo simulations doses in the range 17-20 mg/kg (191). High-dose gatifloxacin-based MDRTB treatment was successful against phenotypically low-level resistant strains in a study with the purpose of identifying fluoroquinolone MIC breakpoints in $7 \mathrm{H} 10$ and BACTEC MGIT 960 and resistance mutations predictive of poor clinical outcome (180). In the same study treatment failure was evident in the presence of gyrA mutations conferring high-level resistance such as D94G. Even if dysglycaemia has not been an issue for levofloxacin as for gatifloxacin, and there may be less problems than for moxifloxacin with regard to QT-prolongation and drug-drug interactions (192), less is known about 
tolerability and safety of higher than recommended doses of levofloxacin $(70,193)$. A randomised ongoing dose-ranging study (OptiQ trial; NCT01918397) evaluating the contribution of levofloxacin to MDR-TB treatment, will present data on correlations of levofloxacin dose increments $(11,14,17$ and $20 \mathrm{mg} / \mathrm{kg}$ ) and potential favourable treatment outcome without increased risk of toxicity (194). Until more evidence on safety and clinical outcome data is available, it may be wise to avoid using levofloxacin in case of low-level resistance mediated by mutations such as A90V, S91P or D94A, even if the MIC value is in the suggested ATU that technically is just below the current critical concentration $(70,92)$. This is also preferable if other equally good drug choices are available or consider switching to moxifloxacin, provided closely monitored side effects (92).

According to the WHO there is no "intermediate" susceptible category applied to TB drugs (59). Nevertheless, a higher clinical breakpoint compared with the critical concentration for moxifloxacin at $1 \mathrm{mg} / \mathrm{l}$ in BACTEC MGIT $960(2 \mathrm{mg} / \mathrm{L}$ in Middlebrook $7 \mathrm{H} 10$ ) has been introduced. The WHO recommends an increased daily dose moxifloxacin of $800 \mathrm{mg}$ for isolates that have elevated MICs above the critical concentration at 0.25 $\mathrm{mg} / \mathrm{L}$ in BACTEC MGIT $960(0.5 \mathrm{mg} / \mathrm{L}$ in Middlebrook 7H10) but below or equal to the clinical breakpoint (59). Even if the clinical evidence for this recommendation is limited and mainly extrapolated from one study (180), this approach is very similar to how the intermediate category "susceptible, increased exposure" is used clinically when set by EUCAST and outlined in the clinical breakpoint tables (www.eucast.org).

There is no obvious reason to believe that the SIR-system would work differently or less well for M. tuberculosis compared to other bacterial pathogens. When applicable for the specific agent and pathogen, the usage of the category "susceptible, increased exposure" (I) can reduce the risk of inadequate treatment and additional acquired drug resistance, therefore worth identifying. The challenge is to gather enough clinical and PK/PD data in order to increase the clinical breakpoint above the ECOFF.

\section{Genotypic testing and prediction of susceptibility}

Mutations in codons 90 and 94 are the most common resistance mutations within the gyrA gene and can be detected by rapid gDST such as Genotype ${ }^{\circledR}$ MTBDRsl $(94,181)$. In a recent systematic review of 19 studies (2223 clinical isolates), the pooled sensitivity and specificity estimates for detection of fluoroquinolone resistance by using Genotype ${ }^{\circledR}$ MTBDRsl version 1.0 compared with pDST for fluoroquinolones, were $85.6 \%$ and 98.5\% (95). In study I sensitivity and specificity for the MTBDRsl version 1.0 to detect resistance mutations using gyrA sequencing as the reference standard, was $96 \%$ and $98 \%$, respectively. The main reason for the lower sensitivity in the systematic review compared with study I is most likely the difference in choice of reference standard (pDST versus sequencing). Phenotypic DST as the reference standard, decreases the sensitivity for targeted gDST since pDST virtually detects all resistance, not only the phenotypical resistance conferred by hot-spot mutations in gyrA as interrogated in MTBDRsl version 1.0. On the contrary, pDST may have difficulties detecting mutations conferring lowlevel resistance for TB drugs exhibiting a close relationship between wild-type and nonwild-type profiles, why a combination of gDST and pDST could be preferred to use for some drugs like the fluoroquinolones. Subgroup analysis of the six studies presenting 
data on the performance of MTBDRsl version 1.0 using only sequencing as reference standard, showed pooled sensitivity and specificity $(95 \%$ confidence interval (CI)) of $99.3 \%(81.2 \%$ to $100.0 \%)$ and $99.3 \%(90.8 \%$ to $100.0 \%)$ for detection of fluoroquinolone resistance, which is in line with the results in study $\mathbf{I}$.

In study I single mutations detected by MTBDRsl version 1.0 correlated to higher MIC ranges for MUT3A-C (codon 94; levofloxacin 2-8 mg/L and ofloxacin 4-16 mg/L, $\mathrm{n}=12$ ) compared with MUT1 (codon A90V; levofloxacin 0.25-2 mg/L and ofloxacin 1-4 mg/L, $\mathrm{n}=9$ ). Potentially, this could be used in clinical practice for early identification of lowand high-level levofloxacin resistance, similar to the use of MTBDRplus for low- and high-level isoniazid resistance. However, the different MIC-distributions of levofloxacin, corresponding to each gyrA mutation, showed an overlap hence not easily identifiable in a recent technical report on genotype-phenotype correlations (59). More data are needed for identification of low- and high-level levofloxacin resistance. Probably, gDST is a better method to identify low-level (A90V) and high-level resistance (codon 94 mutations) than pDST because of the variability in the latter method. The distinction between levels of resistance tended to be more evident for moxifloxacin in the technical report. The definition of two moxifloxacin breakpoints was established to distinguish low-level gyrA mutants (mainly in A90V, S91P and D94A) from high level mutants (most often observed in D94G) and these mutations are all detected by MTBDRsl assays. In study I only one strain harboured MUT3A (D94A) and, in contrast to what was reported for moxifloxacin, conferred high-level phenotypical resistance for both ofloxacin and levofloxacin. In the technical report, the levofloxacin MIC-distributions of D94A mutation in different media is close, both to the MIC-distribution of mutations in codon 90 as well as to the profile of other mutations in codon 94 . This illustrates the difficulties in separating MIC-distributions of different mutations when there is a close phenotypic relationship between low- and high-level resistance.

In a large survey within the 100,000 Genomes Project it was concluded that the predictions of pan-susceptibility from WGS met the WHO standards for new molecular assays for $M$. tuberculosis, with the requirements of more than $90 \%$ sensitivity and $95 \%$ specificity (79). Consequently, in some European countries WGS has started to replace pDST of first-line drugs (195). This is in line with the findings in study II where WGS DST was compared with pDST in BACTEC MGIT 960 for a limited collection of fully susceptible isolates by pDST. No high-confidence drug resistance mutations were found for the first-line drugs. In further support of WGS, a recent investigation showed the feasibility and high accuracy of WGS-based treatment for MDR/XDR-TB and the risk of underestimating resistance by pDST, largely due to poorly defined critical concentrations (196). However, not even WGS is a one-size-fit-all solution given the incomplete understanding of the genotypic basis of phenotypic resistance (197). Additional challenge limits the full use of WGS and refer to systematic errors due to poorly defined breakpoints for $\mathrm{pDST}$ used as the reference standard for WGS validation (171). Until these challenges are solved, individualised TB treatment for drug-resistant TB is best guided by combined gDST and pDST, preferable by using algorithms that emphasis the strength of each technique while including appropriate confirmatory testing when needed to minimise effects of the limitations $(92,196)$. 
In general, less is known about the levels of phenotypical resistance conferred by individual mutations for second-line drugs. One of the objectives in the translational prospective study IV on pulmonary MDR-TB is to correlate the genotype determined by WGS to the level of phenotypic resistance measured as the MIC in broth microdilution, in relation to the individual drug exposures along with treatment outcome data. However, more and larger clinical studies will be needed to elucidate these relationships to be useful for MDR-TB treatment.

\section{Can measurement of drug concentrations be a useful tool in TB treatment?}

\section{Peak levels of first-line drugs below the reference range are common in TB patients}

Several studies mainly from high-endemic countries, have investigated first-line drug exposures during standard TB treatment. Peak drug concentrations below reference range are common e.g. rifampicin concentration $<8 \mathrm{mg} / \mathrm{L}$ has been observed in about $30-80 \%$ of TB patients $(53,135)$. This is in line with the findings in study II conducted in a lowendemic setting where $42 \%(13 / 31)$ of the TB patients had rifampicin peak $\left(\mathrm{C}_{\text {high }}\right)$ concentration below the lower reference range of $8 \mathrm{mg} / \mathrm{L}$ after two weeks treatment. Lower than recommended peak drug concentrations for isoniazid, pyrazinamide and ethambutol were detected in 19\% (6/31), 27\% (7/26) and 16\% (3/19) of the TB patients (Figure 13). No difference was observed in the median dose per $\mathrm{kg}$ bodyweight administered to patients with low versus normal $\mathrm{C}_{\text {high }}$ of the first-line drugs, except for ethambutol (15.8 versus $20.0 \mathrm{mg} / \mathrm{kg}$ ). Estimation of median $\mathrm{AUC}_{0-6 \mathrm{~h}}$ for rifampicin and isoniazid (35 and $17 \mathrm{mg} \cdot \mathrm{h} / \mathrm{L}$, respectively) in study II was performed using noncompartmental analysis based on sparse sampling thus provided crude estimates. Nevertheless, results were in line with previous findings of TB patients receiving standard $\mathrm{TB}$ treatment achieving a median $\mathrm{AUC}_{0-6 \mathrm{~h}}$ for rifampicin of $24.9 \mathrm{mg} \cdot \mathrm{h} / \mathrm{L}$ (198). 


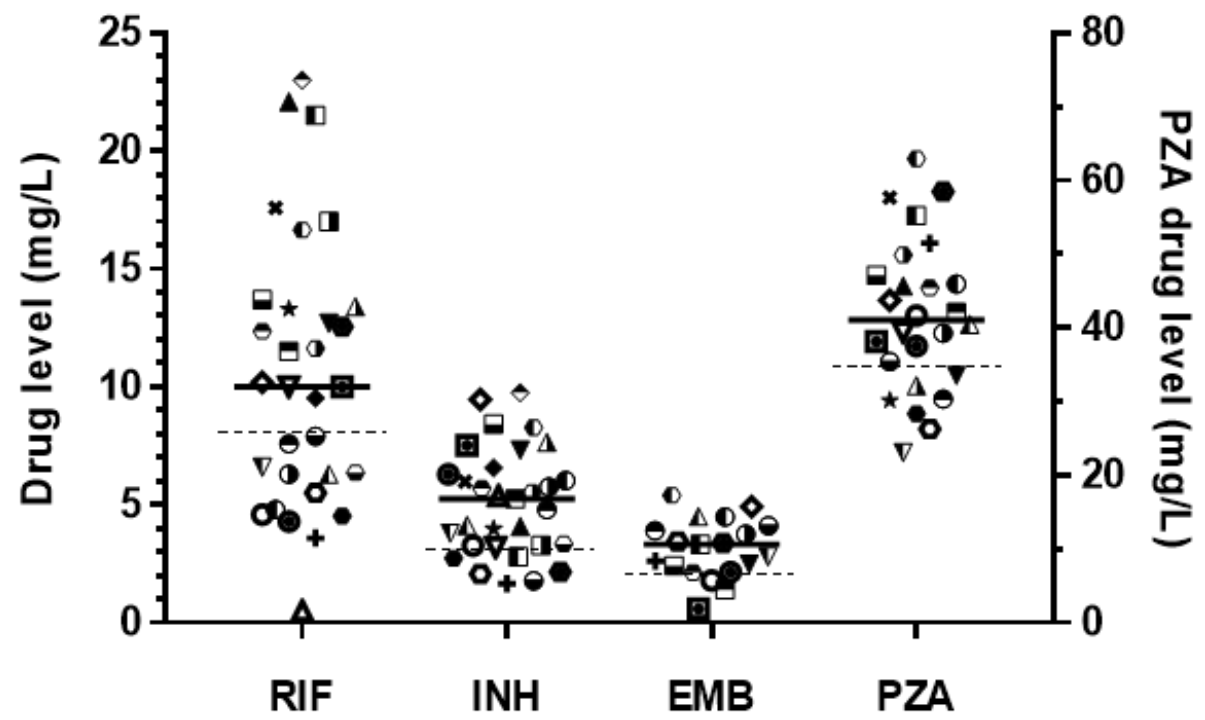

Figure 13. Distribution of $C_{\text {high }}$ for first-line TB drugs at week 2 in patients with active TB. Individual drug levels of rifampicin, isoniazid and ethambutol are plotted against the left y-axis and the right $y$-axis corresponds to the pyrazinamide plasma concentration. Medians are represented by the horizontal bold lines. The horizontal broken lines show the lower reference range for each drug (RIF<8 mg/L, INH<3 mg/L, EMB $<2 \mathrm{mg} / \mathrm{L}$ and $\mathrm{PZA}<35 \mathrm{mg} / \mathrm{L})(53,138)$.

RIF: rifampicin, INH: isoniazid, EMB: ethambutol, PZA: pyrazinamide.

One of few other studies conducted in a low-endemic setting in Denmark included 32 patients on standard therapy against active TB and three patients on treatment for latent tuberculosis (136). This study reported low two hours $\left(\mathrm{C}_{2 \mathrm{~h}}\right)$ drug levels for isoniazid $(<3$ $\mathrm{mg} / \mathrm{L})$ in $71 \%$, rifampicin $(<8 \mathrm{mg} / \mathrm{L})$ in $58 \%$, pyrazinamide $(<20 \mathrm{mg} / \mathrm{L})$ in $10 \%$ and for ethambutol $(<2 \mathrm{mg} / \mathrm{L})$ in $46 \%$ of the participants. Drug concentrations below reference range of both isoniazid and rifampicin were observed in $45 \%(15 / 33)$ of the patients in the Danish study, while this was noted in only $13 \%$ (4/31) of TB patients in study II. Similarly, a meta-analysis covering PK studies of first-line drugs mainly from highburden countries during 1970-2014 reported higher frequency of low $\mathrm{C}_{2 \mathrm{~h}}$ than in study II (rifampicin $67 \%$ versus $42 \%$, isoniazid $43 \%$ versus 19\%) (138). The lower proportion of patients with low drug levels in study II compared with the Danish study and the metaanalysis may have several explanations. The bioavailability may differ as TB patients in study II ingested the drugs under strict fasting conditions aided by DOT on the day of PK sampling and no fixed dose combinations were used. Moreover, the richer PK sampling in study II enabled detection of delayed drug absorption compared with a twohour blood sample. A single two-hour blood sample has been questioned $(53,199,200)$, although it is often used in clinical practice. Pharmacogenetics may differ between settings, where for instance $80-90 \%$ of Chinese and Japanese are fast isoniazid acetylators (homogenous for the wild-type gene $\mathrm{N}$-acetyltransferase 2 (NAT2) involved in isoniazid 
metabolism) meanwhile approximately $40-50 \%$ of whites, blacks and South Indians have this characteristic $(2,201,202)$. The majority of the patients in study II originated from sub-Saharan Africa (19/31, 61\%). Forty-one percent of the study participants were fast acetylators and these patients achieved significantly lower isoniazid peak drug concentrations compared with slow acetylators throughout the period of PK sampling (2, 4,12 weeks). As an example of pharmacogenetic-based therapy, dose adjustments of isoniazid according to NAT2 genotype were performed in a study in Japan. Participants $(\mathrm{n}=172)$ were randomized to either conventional TB treatment or NAT2 genotype-guided treatment with different dosing of isoniazid according to acetylator status (203). Less treatment failures (persistent positive sputum culture or no improvement in chest radiographs after eight weeks) were observed in fast acetylators and fewer adverse events in slow acetylators.

\section{Rifampicin - prone to interactions and a pharmacokinetic challenge}

Rifampicin absorption may be the most variable among the TB drugs and fat-containing food both delays absorption and reduces bioavailability down to two-thirds of the peak drug concentration $(53,204)$. Drug-drug interactions are common for rifampicin, although they rarely affect the drug exposure of rifampicin itself $(62,205)$. One mechanism behind this is that rifampicin is a potent inducer of several drug-metabolising enzymes within the cytochrome P450 system (205). The distribution of drug concentrations in study II was particularly wide for rifampicin (Figure 14). The highestto-lowest ratio of rifampicin $\mathrm{C}_{\text {high }}$ was $>40$-fold $\left(\mathrm{C}_{\text {high }}\right.$ range $0.5-23 \mathrm{mg} / \mathrm{L}$ ) compared with 3 -fold for isoniazid adjusted for the acetylator status (fast acetylators; $\mathrm{C}_{\text {high }}$ range 1.8-6 $\mathrm{mg} / \mathrm{L}$, slow acetylators; 3.3-9.8 mg/L). The highest-to-lowest dose $(\mathrm{mg} / \mathrm{kg}$ ) was only twofold for both rifampicin and isoniazid. In a large prospective cohort study conducted in a high-burden setting, the ratios of the highest-to-lowest peak concentrations for rifampicin was $>100$-fold and for isoniazid 31-fold, the latter not adjusted for acetylator status (127). Moreover, in study II individual rifampicin concentrations displayed more fluctuations over time compared with isoniazid measured at week 2,4 and $12(p<0.001)$. The median $\mathrm{C}_{\text {high }}$ of isoniazid was significantly lower for fast acetylators compared with slow acetylators $(3.2 \mathrm{mg} / \mathrm{L}(\mathrm{n}=11)$ versus $6.2 \mathrm{mg} / \mathrm{L}(\mathrm{n}=16), \mathrm{p}=0.002)$ at week 2 and likewise at week 4 and 12 . 

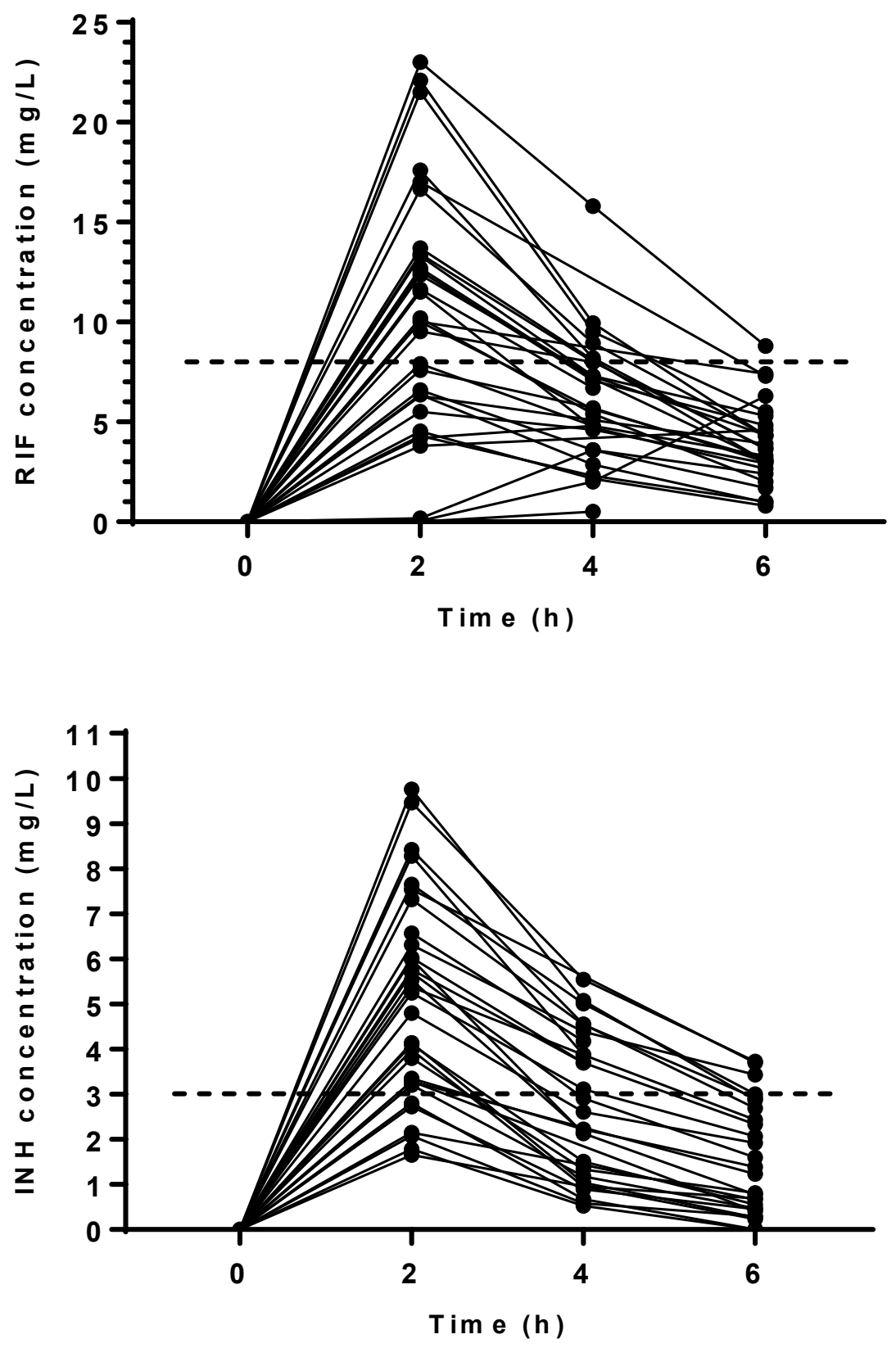

Figure 14. Plasma concentrations of rifampicin (upper graph) and isoniazid (lower graph) in patients with active TB at week 2 at $0,2,4$ and 6 hours. The dotted horizontal lines represent the lower limit of the reference range for the drugs (RIF; $8 \mathrm{mg} / \mathrm{L}, \mathrm{INH} ; 3 \mathrm{mg} / \mathrm{L}$ ).

RIF: rifampicin, INH: isoniazid 
Monitoring of peak drug concentrations early in the intensive phase of TB treatment is most important for meaningful dose-response adjustments. The commonly used time point chosen for PK sampling, is at two weeks after treatment initiation at expected time of steady state of first-line drugs $(53,206)$. Rifampicin clearance is enhanced after repeated doses due to autoinduction of metabolising hepatic enzymes $(207,208)$. In addition, a decrease in oral bioavailability of standard dosing has been reported (reduction from more than $90 \%$ to $\sim 70 \%$ ) within the first three weeks of treatment attributed to autoinduction of non-hepatic pre-systemic elimination (i.e. increased "first-pass" effect) (208). Moreover, rifampicin exhibits nonlinear increase in exposure when higher doses are used (56), with a suggested dose-dependent bioavailability due to saturable nonhepatic pre-systemic elimination in addition to a capacity-limited biliary excretion (207, 209). This dose-dependent characteristic leads to more than proportional increase in drug exposure when using higher doses of rifampicin, which may jeopardize safety such as the risk for hepatotoxicity. In contrast, the changes in time-dependent elimination of the drug (pre-hepatic and hepatic autoinduction) leads to lower exposures over time with the risk of suboptimal treatment (209). Considering higher doses of rifampicin being the future in TB treatment, these complex phenomena need to be accounted for when TDM is used as the exposures may be highly unpredictable. Recently, a population pharmacokinetic model accounting for autoinduction, concentration-dependent clearance and dosedependent bioavailability was presented and enables prediction of rifampicin concentrations at doses up to $40 \mathrm{mg} / \mathrm{kg}$, which could be a useful tool in TDM management of rifampicin (209).

\section{Does low drug exposure have implications for treatment outcome?}

Several attempts to correlate suboptimal drug exposure to poor TB outcome have been made and as shown in Table 8 these show conflicting results as 12 of 25 studies were able to show such association.

In a prospective study in South Africa drug exposure at two months treatment in relation to clinical outcome was investigated for 142 patients with drug-susceptible pulmonary TB (127). Pharmacokinetic thresholds of 24-hour AUC associated with poor treatment outcome were identified and among the patients with low pyrazinamide $\left(\mathrm{AUC}_{0-24 \mathrm{~h}} \leq 363\right.$ $\mathrm{mg} \cdot \mathrm{h} / \mathrm{L})$ or rifampicin $\left(\mathrm{AUC}_{0-24 \mathrm{~h}} \leq 13 \mathrm{mg} \cdot \mathrm{h} / \mathrm{L}\right)$ drug exposure $17 / 60$ relapsed compared with $0 / 64$ of patients without. A pyrazinamide peak concentration below $58.3 \mathrm{mg} / \mathrm{L}$ was the best predictor to identify patients $(11 \%, 15 / 142)$ who did not sputum culture convert after two months treatment. In study II all but two patients (12/14), who could be evaluated for two-month sputum culture conversion, had pyrazinamide concentration below this suggested threshold. However, only one patient in study II remained culture positive at month two. The poor long-term treatment outcome also differed between the studies with a rate of $25 \%$ ( 19 relapsed, 15 died, and 2 had therapy failure) in the South African study compared with $6 \%$ ( 2 relapsed, no deaths or therapy failures) in study II. Unfortunately, data were too limited in study II to predict 24-hour AUC for first-line drugs and the sample size too small for further analysis of treatment outcome. Reasons for the discrepancies between the studies may be several such as differences in disease burden of the cohorts or the marked difference in the proportion of patients with prior 
TB, which was reported for $64 \%$ of the patients in the study in South Africa (127) compared with only $16 \%(5 / 31)$ in study II.

The new tentative PK-targets by Pasipanodya and colleagues need to be evaluated in prospective cohorts of previously untreated TB cases with well-defined disease burden, as their generalisability is limited by the high proportion of patients who were treated for recurrent TB and 50\% received streptomycin in addition to first-line oral drugs (127). Recently, the tentative $\mathrm{AUC}_{0-24 \mathrm{~h}}$ and $\mathrm{C}_{\max }$ thresholds were applied to a large study cohort of new cases of HIV-positive drug-susceptible pulmonary TB (145). In this prospective study the proposed cut-offs by Pasipanodya and co-authors were not predictive of unfavourable treatment outcome (although relapses were not assessed) or delayed sputum culture conversion. When the lower limits of the commonly used drug reference ranges were applied (rifampicin $<8 \mathrm{mg} / \mathrm{L}$, isoniazid $<3 \mathrm{mg} / \mathrm{L}$, pyrazinamide $<20 \mathrm{mg} / \mathrm{L}$, ethambutol $<2 \mathrm{mg} / \mathrm{L}$ ), low concentrations of isoniazid and rifampicin were associated with delayed sputum culture conversion and in combination were a risk factor for unfavourable treatment outcome. This illustrates that PK targets may differ between certain TB cohorts. In line with this observation, different thresholds for rifampicin $(<3.01 \mathrm{mg} / \mathrm{L})$ and pyrazinamide $(<38.1 \mathrm{mg} / \mathrm{L})$ were associated with treatment failure and death in a cohort of children with high proportion of extra-pulmonary TB in a large prospective study with high rate of HIV co-infection (210). In line with treatment of bacterial infection in neutropenic patients with increased dosing of antibiotics, adequate drug exposure is important for TB patients with HIV co-infection as their immune defence against TB is compromised. A higher dose of rifampicin was reported to reduce mortality in a small study of TB meningitis patients and survival was observed to be closely related to drug exposure (211). In this TB cohort, plasma $\mathrm{AUC}_{0-6 \mathrm{~h}}$ and $\mathrm{C}_{\max }$ of approximately $70 \mathrm{mg} \cdot \mathrm{h} / \mathrm{L}$ and $22 \mathrm{mg} / \mathrm{L}$ during the first critical days of treatment were suggested as minimum target values. In comparison, the median rifampicin $\mathrm{AUC}_{0-6 \mathrm{~h}}$ in study II was $35 \mathrm{mg} \cdot \mathrm{h} / \mathrm{L}$ (IQR; 22-47), although determined after two weeks of treatment, this is half of the proposed PK-target of $70 \mathrm{mg} \cdot \mathrm{h} / \mathrm{mg} / \mathrm{L}$. Even if two patients experienced relapse in study II the treatment success rate according to the WHO was $100 \%$ in this mixed population of pulmonary and extra-pulmonary TB. However, none of the patients in study II had TB in the central nervous system. One of the reasons to the higher drug exposure needed in the TB meningitis cohort is that despite wide distribution of rifampicin in tissues the drug exhibits poor penetration to the central nervous system. When using standard doses of rifampicin orally and intravenously a concentration of about 0.5 and $0.73 \mathrm{mg} / \mathrm{L}$, respectively can be reached in normal cerebrospinal fluid, but may be increased 4- to 8-fold if the meninges are inflamed $(33,212)$.

A study in HIV-prevalent setting in Botswana with study participants consisting mainly of new pulmonary TB cases showed that pyrazinamide peak concentration below 35 $\mathrm{mg} / \mathrm{L}$ increased the risk of poor treatment outcome regardless of HIV infection (161) and similar result was recently found by Rockwood and co-authors (149). In study II, 30\% (4/13) of the patients with available data for pyrazinamide peak concentration and who were evaluable for two-month sputum culture conversion, exhibited low pyrazinamide peak concentration $(<35 \mathrm{mg} / \mathrm{L})$ and in this group of four patients the only two-month nonconverter in the study was found. Of note, the small sample size in study II prevented any statistical analysis of correlations between drug exposure and treatment outcome. 
Furthermore, 42\% (10/24) of the patients with pulmonary TB were not evaluable for twomonth sputum culture conversion due to mainly inability to provide sputum but also due to missing sample, culture negative pulmonary $\mathrm{TB}$ at baseline or contamination of the culture tube by other bacteria.

In conclusion, there may be several reasons for conflicting results regarding the clinical importance of variability in first-line drug exposure. First, many studies are underpowered to detect associations between drug exposure and treatment outcomes (135). Second, measuring only $\mathrm{C}_{2 \mathrm{~h}}$ may miss peak drug concentration due to delayed absorption and AUC may be more closely related to treatment response than peak concentration. Third, pre-determined cut-off values for peak concentrations of TB drugs used to classify patients as having either low or high concentrations may be misleading. These lower limits are based mainly on normal drug ranges achieved in healthy volunteers and in TB patients not well validated against clinical outcome $(127,135)$. Fourth, few clinical studies include MIC data which limits deeper analysis of tentative PK/PD targets accounting for drug-pathogen interaction. The same PK/PD-targets may not be valid for all TB patients but could depend on TB manifestation (145), disease severity and host immune status. Fifth, evaluation of TB treatment is difficult and in PK studies two-month sputum culture conversion is often used as a crude surrogate marker of treatment outcome instead of assessment of sterilising capacity best reflected by the occurrence of TB relapse. Finally, evaluation of $\mathrm{PK} / \mathrm{PD}$ studies in TB is challenging as combination therapy is mandatory. It is therefore difficult to elucidate the PK/PD relationship for each drug as well as the influence of potential antagonism and synergism between first-line drugs $(149,210)$.

\section{Are the MICs of TB drugs of importance for treatment outcome?}

Reliable DST of $M$. tuberculosis is important for treatment outcome as recently shown in a multi-centre study conducted in high-burden countries based on a mixed population of drug-susceptible and drug-resistant TB patients $(\mathrm{n}=634$ of which 193 patients had MDR/XDR-TB) (213). Regardless of HIV-status inaccurate DST results increased mortality during treatment almost two-fold compared with patients that had concordant results. Pyrazinamide pDST is rarely performed in high-burden settings, which has consequences for treatment outcome since a large meta-analysis of MDR-TB treatment recently showed lower mortality if pyrazinamide was included in the regimen provided the isolate was susceptible (66). In line with this, a retrospective Swedish study over two decades found that pyrazinamide was associated with shorter time to sputum culture conversion (median 27 days) for patients receiving pyrazinamide in their MDR-TB regimen and had a pDST result showing pyrazinamide susceptible MDR-TB (214). Cavitary pulmonary TB, pre/XDR-TB and HIV were not associated with time to sputum culture conversion.

Determination of MIC provides refined information on the degree of resistance in contrast to binary routine pDST, often based on poorly validated critical concentrations. The use of individual MICs has been questioned in a recent editorial by Mouton and co-authors, stressing the high variability of MIC determination and whether there is a true biological variability within the wild-type population (215). However, MIC values have shown to be of importance in several studies. In a study in South Africa of 36 pulmonary TB 
patients whereof $65 \%$ had prior TB, higher MICs of isoniazid and rifampicin (although below the critical concentrations) were predictive of poor treatment outcome (216). Moreover, Colangeli and co-authors explored bacterial factors predictive of relapse after TB therapy of drug-susceptible TB (217). In this retrospective study, TB patients who experienced relapse were shown to have pre-treatment isolates with increased MIC values but below current critical concentrations, for isoniazid and rifampicin compared with the isolates collected from the cured and relapse-free patients. Increments of MIC values of either rifampicin or isoniazid were associated with a higher risk of relapse even after adjusting for other risk factors linked to relapse. In another retrospective study conducted nationwide in Sweden, increments of fluoroquinolone MICs were significantly correlated to unsuccessful treatment outcome in patients treated for MDR-TB (63). Interestingly the MICs in the study were below the current critical concentrations. Clearly, data on MIC values can be of importance but due to the technical variability they should in general be thought as levels of susceptibility corresponding to successful therapy rather than as absolute thresholds (84). Inclusion of quality control isolates and sometimes repeat testing are of importance to get high quality results.

It is not uncommon for M. tuberculosis to have MICs between the ECOFFs and the critical concentrations representing strains with low-level drug resistance. Such isolates are not detected by routine pDST using current critical concentrations, as these breakpoints sometimes split the MIC-distributions corresponding to strains with mutations conferring low-level resistance. This is illustrated in study I for fluoroquinolones and certain gyrA mutations. Access to detailed DST of first- and second-line drugs is needed to improve outcomes in patients with MDR-TB and preXDR/XDR-TB $(66,180)$. The most promising approach to reach this goal is by WGS, because it is more rapidly available than pDST results. However, much work remains until this approach is feasible in TB and also affordable in high-burden countries.

\section{The role of MIC in PK/PD-indices and the implications for therapeutic drug monitoring in tuberculosis treatment}

Provided low intra-laboratory variability, determination of individual MIC allows for identification of wild-type, intermediately susceptible and resistant strains, in accordance with Mouton et al. (215). The SIR-classification is beneficial for TDM as single MIC determinations are less accurate for dose-guidance than SIR defined cut-offs $(92,215)$. However, for most of the TB drugs there is a lack of definitions of ECOFFs and insufficient data of the different MIC-distributions corresponding to low- and high-level resistance, to be able to establish breakpoints according to the SIR-classification (75). Until the relationship between genotype-phenotype and clinical breakpoints have been properly defined in a reference pDST method, MIC-determination can be a useful clinical tool to better define the level of drug susceptibility, particularly for MDR-TB cases. Furthermore, the complexity of TB treatment, especially current regimens against MDR$\mathrm{TB}$, makes it very difficult to set clinical breakpoints that include evaluation of clinical outcome data of a single drug versus the level of susceptibility. Hence, the remaining major tools for setting clinical breakpoints, are wild-type profiles and PK/PD calculations in relation to tentative PK/PD targets (mainly AUC/MIC, $\mathrm{C}_{\max } / \mathrm{MIC}$ ) derived from preclinical studies commonly based on monotherapy (187). In addition, determination of 
non-wild-type profiles corresponding to different resistance mutations particularly those with low-level resistance, combined with PK/PD calculations may identify isolates that could be treated if drug exposure is increased compared with standard dose.

The importance of including individual MICs, rather than a single, arbitrary MIC representing literature derived modal MIC of a wild-type distribution, to enable adequate TDM has been emphasized by field experts. In recent reviews, the lack of such data in clinical PK studies of TB drugs has been underlined $(121,141)$. By simple mathematics it is evident that with the same drug exposure for concentration-dependent TB drugs, the achieved exposure-response ratio is highly dependent on the MIC. For instance, for a TB patient with a rifampicin $\mathrm{C}_{\max }$ of $8 \mathrm{mg} / \mathrm{L}$ (the lowest level of the reference range) and an M. tuberculosis isolate with MIC of $1 \mathrm{mg} / \mathrm{L}$ in BACTEC MGIT the current critical concentration of rifampicin, will have a rifampicin $\mathrm{C}_{\max } / \mathrm{MIC}$ ratio of 8 . On the other hand, a patient with the same rifampicin $\mathrm{C}_{\max }$ of $8 \mathrm{mg} / \mathrm{L}$ but with a highly susceptible isolate e.g. MIC of $0.016 \mathrm{mg} / \mathrm{L}$ in BACTEC MGIT as occurred in study II, will reach a ratio of rifampicin $\mathrm{C}_{\max } / \mathrm{MIC}$ of 500. These examples stress the importance of taking the individual MIC into consideration, if feasible, when decision is made to use TDM for individual based dose adjustments in TB treatment. Taking the variability of the method into account, a two-fold dilution margin or more may be used for individual target attainment calculations, since a small difference in the denominator (MIC) has greater impact on the ratio (215). Determination of MICs in BACTEC MGIT 960 in study II exhibited low intra-laboratory variability and the agreement with the MYCOTB (Sensititre ${ }^{\mathrm{TM}}$ ) method was within \pm 1 two-fold MIC dilution steps for $>95 \%$ of the observations. The inclusion of quality control data is a strength of study II as this is rare in MIC studies within the TB field as illustrated in a recent WHO review (59). A wide distribution was observed in study II for rifampicin $\mathrm{AUC}_{0-6 h} / \mathrm{MIC}$ and $\mathrm{C}_{\text {high }} / \mathrm{MIC}$ and to a lesser extent for isoniazid as illustrated in Figure 15. 


\section{$\mathrm{AUC}_{0-6 \mathrm{~h}} / \mathrm{MIC}$ for RIF and INH}

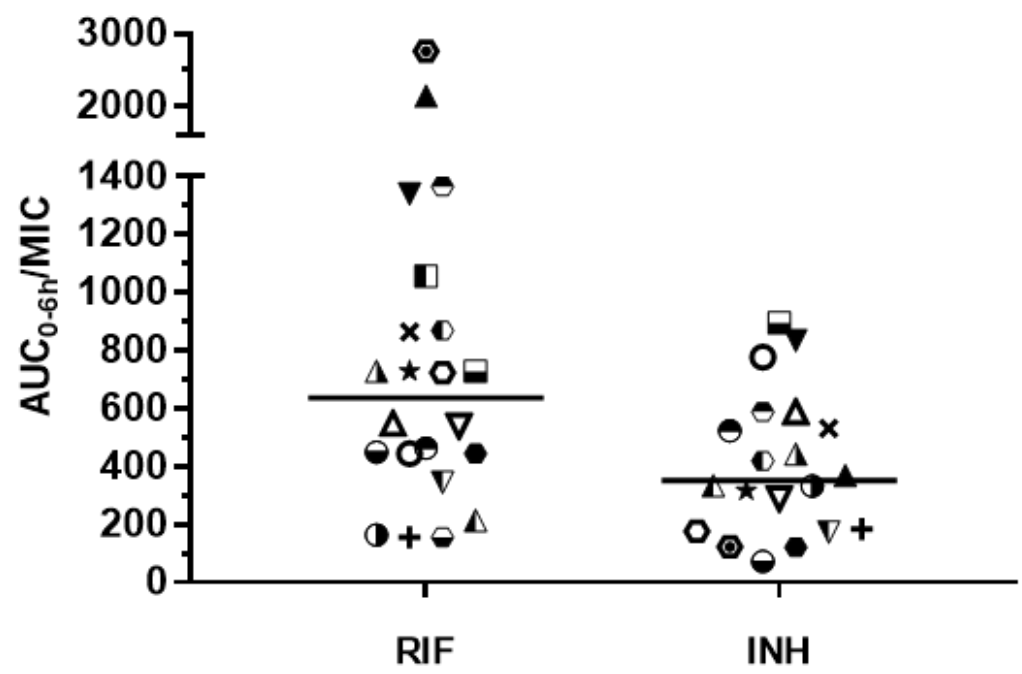

\section{$\mathrm{C}_{\text {high }} / \mathrm{MIC}$ for RIF and INH}

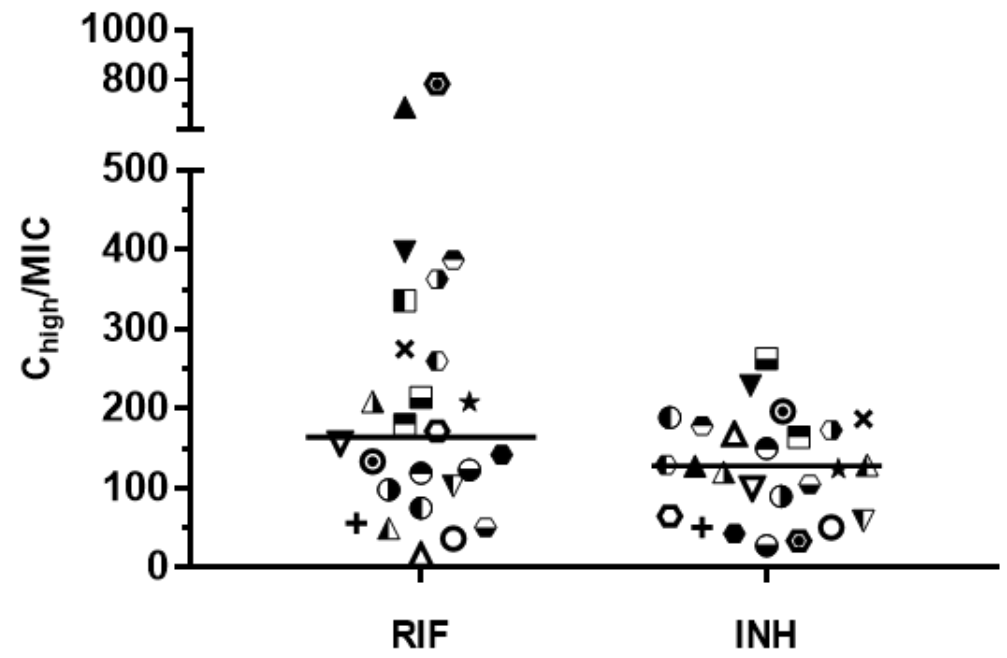

Figure 15. Distribution of $\mathrm{AUC}_{0-6 h} / \mathrm{MIC}$ (upper graph) and $\mathrm{C}_{\text {high }} / \mathrm{MIC}$ (lower graph) for rifampicin $(n=22, n=26)$ and isoniazid $(n=20, n=25)$ at week 2 in patients with active TB. For each individual patient, the same dot style is used throughout for all drugs. Medians are presented as horizontal bold lines. The median $\mathrm{AUC}_{0-6 h} / \mathrm{MIC}$ ratio and $\mathrm{C}_{\text {high }} / \mathrm{MIC}$ ratio for rifampicin was 636 and 164, and for isoniazid 351 and 128 respectively.

RIF: rifampicin, INH: isoniazid. 
In Figure 16, the exploratory analysis of composite of rifampicin and isoniazid $\mathrm{C}_{\text {high }} / \mathrm{MIC}$ ratios is displayed for the 25 of the TB patients in study II who had peak drug concentrations and MICs for both rifampicin and isoniazid. These composite ratios of the core drugs differed up to almost 10 -fold (range 88-819, median 269) between TB patients. There were three patients with unfavourable treatment outcome in the study (two with relapse and one with delayed sputum culture conversion beyond two-month treatment). The only patient with pulmonary TB that experienced recurrent TB (1/24) and the only patient who failed to sputum culture convert after two months treatment $(1 / 14)$, were in the lower end of the composite-ratio $(<170)$. These two patients were among the four patients in the study that had TBscore II $>2$ points and most likely these two patients would have benefit from early dose-guidance by TDM. The third patient in the study who suffered unfavourable treatment outcome, was the only patient in this HIV-negative study cohort with known impaired immune defence. This patient experienced TB relapse in a joint prosthesis despite drug concentrations at steady state well within normal range for all first-line drugs (rifampicin $17.6 \mathrm{mg} / \mathrm{L}$, isoniazid $6.0 \mathrm{mg} / \mathrm{L}$, pyrazinamide $57.7 \mathrm{mg} / \mathrm{L}$, ethambutol discontinued before PK sampling). Notably, this patient dropped in rifampicin peak concentration over time down to $4.5 \mathrm{mg} / \mathrm{L}$ at week 12 . 


\section{Composite of RIF $\mathrm{C}_{\text {high }} / \mathrm{MIC}$ and INH $\mathrm{C}_{\text {high }} / \mathrm{MIC}$ ratios}

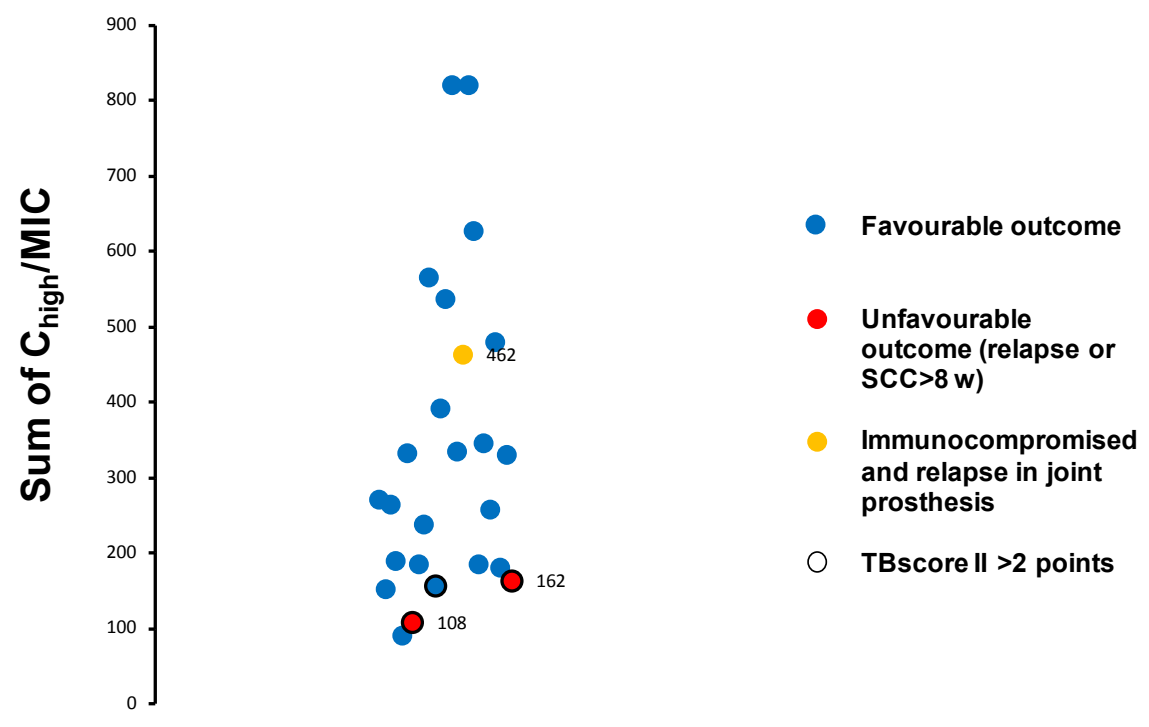

Figure 16. Dot plot of an exploratory analysis of the composite of rifampicin and isoniazid $\mathrm{C}_{\text {high }} / \mathrm{MIC}$ for TB patients with drug-susceptible pulmonary $(\mathrm{n}=21)$ or extra-pulmonary $(n=4)$ TB in study II. Patients with favourable versus unfavourable treatment outcome are discerned by different markers. Patients with higher clinical scoring according to TBscore II are identified by a circle with marked edge.

RIF: rifampicin, INH: isoniazid, SCC: sputum culture conversion, w: weeks.

In PK/PD-studies total (unbound and bound) drug concentration is usually measured and serves as a proxy for the drug concentration at the site of the infection. Albeit, only the free unbound portion of the drug is active in the tissue, which among first-line drugs affects rifampicin ( $\sim 80 \%$ protein binding) the most $(207)$, as outlined for PK/PD parameters in study II in Table 9. 
Table 9. Rifampicin and isoniazid PK/PD parameters of the study participants in study II. Data are presented as median and IQR.

\begin{tabular}{|c|c|c|}
\hline Parameter & Rifampicin & Isoniazid \\
\hline Drug doses, mg/kg & $9.3(8.3-10.1), n=31$ & $5.0(4.3-5.1), n=31$ \\
\hline $\mathrm{C}_{\text {high }} / \mathrm{MIC}$ & 164 (92-290), n=26 & $128(62-176)^{\star \star}, n=25$ \\
\hline$f \mathrm{C}_{\text {high }} / \mathrm{MIC}$ & $33(19-58)^{*}, n=26$ & $128(62-176)^{\star \star}, n=25$ \\
\hline$A \cup C_{0-6 h}, m g \cdot h / L$ & $35(22-47), n=24$ & $17(8-27), n=23$ \\
\hline $\mathrm{AUC}_{0-6 \mathrm{~h}} / \mathrm{MIC}$ & $636(419-915), n=22$ & $351(178-574)^{\star *}, n=20$ \\
\hline$f A \cup C_{0-6 h} / \mathrm{MIC}$ & $128(84-183)^{*}, n=22$ & $351(178-574)^{\star *}, n=20$ \\
\hline
\end{tabular}

${ }^{*}$ Calculations based on free $(f)$ drug concentrations using protein binding level of $80 \%$ for rifampicin (207).

${ }^{\star *}$ Free drug concentrations of isoniazid were measured, and the drug is regarded as unbound in plasma (218).

Ideally, clinicians should be able to measure PK/PD indices in vivo at the site of the infection rather than to rely on surrogate markers including PK/PD indices derived from in vitro and murine models. However, this is not feasible in clinical practice and no methods including clinical evaluation, are free from methodological variability, which is important to keep in mind. Experts in the field do agree that in the absence of clinical exposure-response data for some TB drugs, drug exposure relative the MIC provides an important correlate of activity. Hence, the distribution of both MICs and PK parameters for the individual drugs amongst TB patients are useful when discussing optimal dose (70). A variability in both of these measurements is observed (e.g. geographical variation, different cohorts of patients), which are often challenging to fully understand in terms of clinical importance. However, there are evidence amongst TB patients with $M$. tuberculosis isolates susceptible to TB drugs (e.g. first-line core drugs or fluoroquinolones), but with increased MIC levels, to be associated with poor treatment outcomes $(63,217)$. This underscores the value of including individual MIC determination in clinical studies and in personalised medicine guided by TDM, at least until current breakpoints have been better defined.

\section{Individualised based treatment of TB}

TDM in TB treatment may be of most benefit in patients with malabsorption, HIV coinfection, diabetes, risk of drug-drug interactions, treatment failures, delayed sputum culture conversion and drug-resistant TB, but there are more to TDM than this. There are several ongoing efforts to optimise treatment of drug-susceptible TB by increasing doses. TDM can be a useful tool towards the goal of shorter relapse-free TB treatment. Higher doses of rifampicin are presently investigated and due to nonlinear kinetics at higher doses the drug exposure increases more than proportional (209), which highlights the need of drug concentration measurements. Furthermore, optimised TB treatment with more rapid sputum culture conversion decreases the time of contagiousness.

Suboptimal drug concentrations due to PK variability independent of non-adherence, have been associated with acquired drug resistance in in vitro models and with poor treatment outcome in prospective clinical studies $(127,161,219)$. Many of the factors 
associated with PK-variability are difficult to change, and individualised dosing for TB may be more effective than standardised dosing as used in directly observed therapy programs (44). There are varying opinions about cost-benefit of using TDM (139), but the consequences of treatment failure and acquired drug resistance are not only devastating for the patient but also place a heavy burden on the public health system in terms of resources required and costs (42). To quote a field expert, instead of guessing in the dark, clinicians can switch on the light provided by TDM and make clinical decisions with more precision (140). Figure 17 visualises how TDM potentially can be used in clinical settings.
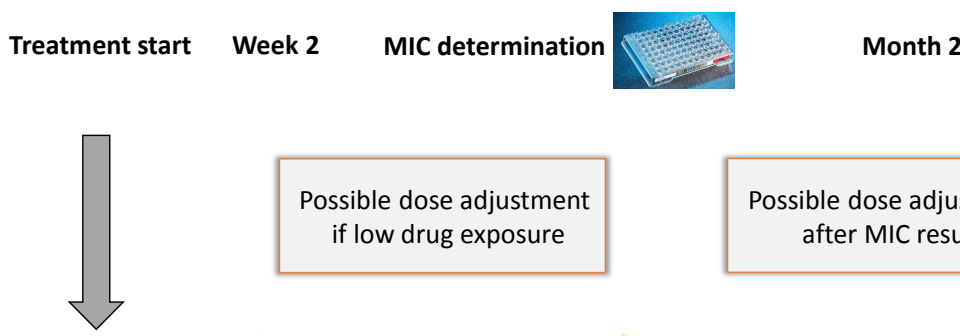

\section{if low drug exposure}

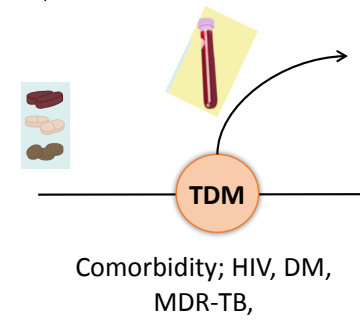

Drug interactions, malabsorption
Verification if dose adjustment was adequate
Possible dose adjustment after MIC results

Adverse events ANY TIME

OR

Failure to SCC

Figure 17. Illustration of TDM for tuberculosis treatment in clinical practice. Blood for drug concentration analysis is collected under fasting conditions at steady state (typically at 2 weeks) using limited sampling strategy ( 2 hours after drug intake for most TB drugs and if feasible also at 4 and 6 hours). Drug concentrations are compared to reference ranges, and if available, the individual MICs and result of genotypic DST. If used, the MIC value should be regarded in the context of plausibility of clinical success by using the planned drug regimen. The site of infection (e.g. TB meningitis, TB pericarditis), the disease severity (e.g. cavitary disease, miliary TB, critically ill) and the host immune defence (e.g. HIVIAIDS, immunocompromised patients) must be considered as these conditions may need increased drug exposure in relation to bacterial susceptibility level. If dose adjustments are performed, a follow-up TDM is recommended 1-2 weeks later. 
In MDR-TB treatment, a TDM-strategy is expected to be of even higher value (92), since the cure rate is nearly half of what is seen in drug-susceptible TB (55\% versus $82 \%)$, the treatment is much longer with in general less efficacious drugs and strongly compromised by side effects (3). However, recent clinical trials and the inclusion of bedaquiline in the regimen may increase the cure rates from empirical regimens. A study in Tanzania on drug levels in MDR-TB treatment observed low concentration of crucial drugs like levofloxacin, in approximately half of the patients (220), although only $\mathrm{C}_{2 \mathrm{~h}}$ blood samples were collected. In a retrospective study in Korea among pulmonary TB patients, the frequency of low drug concentrations of second-line drugs was substantial (221). Unfortunately, the study could not fully evaluate MDR-TB treatment efficacy and the blood sampling interval for drug concentration analysis was wide. Thus, the peak level of each TB drug could not be adequately assessed. The importance of integrating MIC, PK/PD and TDM for optimisation in treatment of MDR-TB has recently been highlighted (222) and numerous knowledge gaps exist concerning lack of PK/PD data for the majority of drugs used in MDR-TB (70).

Study IV (Figure 18) is a prospective cohort study in China and presents a comprehensive translational approach to TDM studies in MDR-TB where drug exposure, individual MIC determination and WGS will be integrated and correlated to time to culture positivity, sputum culture conversion and clinical treatment outcome according to the WHO criteria. The study has recently been closed for recruitment after enrolling 30 patients and data are currently being analysed. Hopefully, study IV will provide useful information regarding drug exposures and treatment response in MDR-TB patients. 


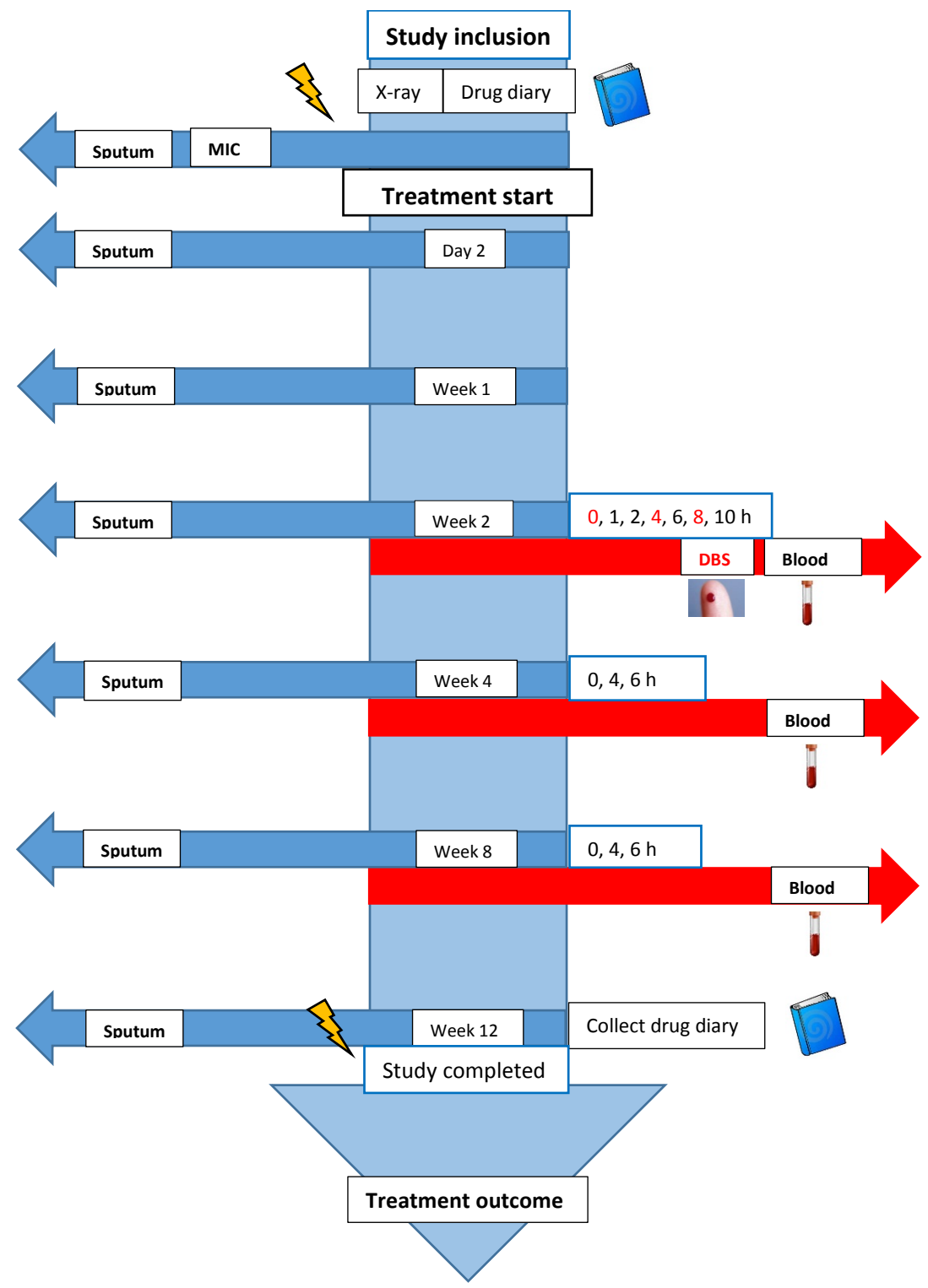

Figure 18. Study outline of study IV. 
Even though TDM is not likely to be cost-effective for indiscriminate use, certain groups of patients and individuals that would gain the most of using TDM should be identified. Immunocompromised and patients with diabetes are most likely among these as are patients with more severe TB disease. To be able to establish PK/PD indices and targets needed for meaningful TDM in TB, it is important to explore and gather as much data as possible regarding PK- and PD-related parameters - without neglecting the shortcomings of today's methods. Important pieces of knowledge can be gained by exploring treatment response to the distribution of PK parameters in relation to individual MICs in different TB cohorts. Even so, in cohorts of drug-susceptible TB as performed in study II, conducted in a low-endemic setting with less disease burden compared with highendemic settings (measured by TBscore II). In this study $16 \%(5 / 31)$ had cavitary disease and $6 \%(2 / 31)$ of the patients experienced relapse, but the treatment success rate according to the WHO criteria was $100 \%$. PK thresholds for efficacy will vary with different forms of TB (e.g. meningitis, pericarditis) and disease severity (e.g. cavitary disease). Moreover, the new up-coming optimised treatments using higher drug doses needs revised thresholds and subsequently identification of PK/PD targets.

\section{Tuberculosis drug activity assay - an alternative tool in therapeutic management for dose-guidance of rifampicin in low-resource settings?}

High-endemic TB areas are mainly situated in low-resources countries (Figure 1), where facilities for drug concentration analysis are limited. Moreover, measurement of drug concentrations for TDM during TB treatment is expensive even in high-resource countries, and thus rarely an option in resource-limited areas. If drug concentration analysis cannot be done, the tuberculosis drug activity assay (TDA) in blood samples from patients on TB drugs can be determined by measuring patient plasma's ability to inhibit growth of M. tuberculosis in vitro (223). Such assays are not unique and have been used before and are generally called biological assays to determine drug concentrations. Heysell and co-authors observed a statistically significant correlation between TDA and drug level and drug MIC, particularly for rifampicin and isoniazid, whereas the drug effect of pyrazinamide and ethambutol was negligible. A TDA ratio was defined as the time to detection in BACTEC MGIT system of plasma-cocultured M. tuberculosis relative the time to detection of control growth. Low TDA $(\leq 2.0)$ was linked to lower $\mathrm{C}_{2 \mathrm{~h}}$ levels of rifampicin and isoniazid compared with TDA levels above 2.0 and additionally, very low TDA $(\leq 1.5)$ corresponded to a trend toward lack of cure (223).

In line with Heysell and co-authors (223), significant correlation, although low, was observed in study III between exact levels of TDA and rifampicin concentrations $(\mathrm{r}=0.54 ; \mathrm{p}=0.002$; Spearman's rank correlation test). Further, TDA $<1.5$ correlated to lower rifampicin concentrations (Figure 19) and as displayed in Figure 20, TDA was significantly lower in TB patients with rifampicin levels below $8 \mathrm{mg} / \mathrm{L}$ compared with patients exceeding $8 \mathrm{mg} / \mathrm{L}$. In contrast to previous findings, no correlation was observed between TDA and isoniazid concentrations as median TDA was similar in patients with isoniazid concentrations below or above the lower limit of the reference range (1.64 $(1.44-1.69)$ versus $1.52(1.35-1.71), p=0.64)$ at $3 \mathrm{mg} / \mathrm{L}$. No correlation was found in study III between TDA and levels of pyrazinamide or ethambutol, which is in line with the previous study (223). An explanation for this could be that pyrazinamide is inactive at the 
$\mathrm{pH}$ of standard Middlebrook media in BACTEC MGIT and ethambutol is largely bacteriostatic. Previously, an association has been observed between TDA $\leq 1.5$ and a lower degree of sputum smear conversion in TB patients on first-line regimen (223). The limited sample size in study III precluded analysis of sputum culture conversions in relation to TDA levels.

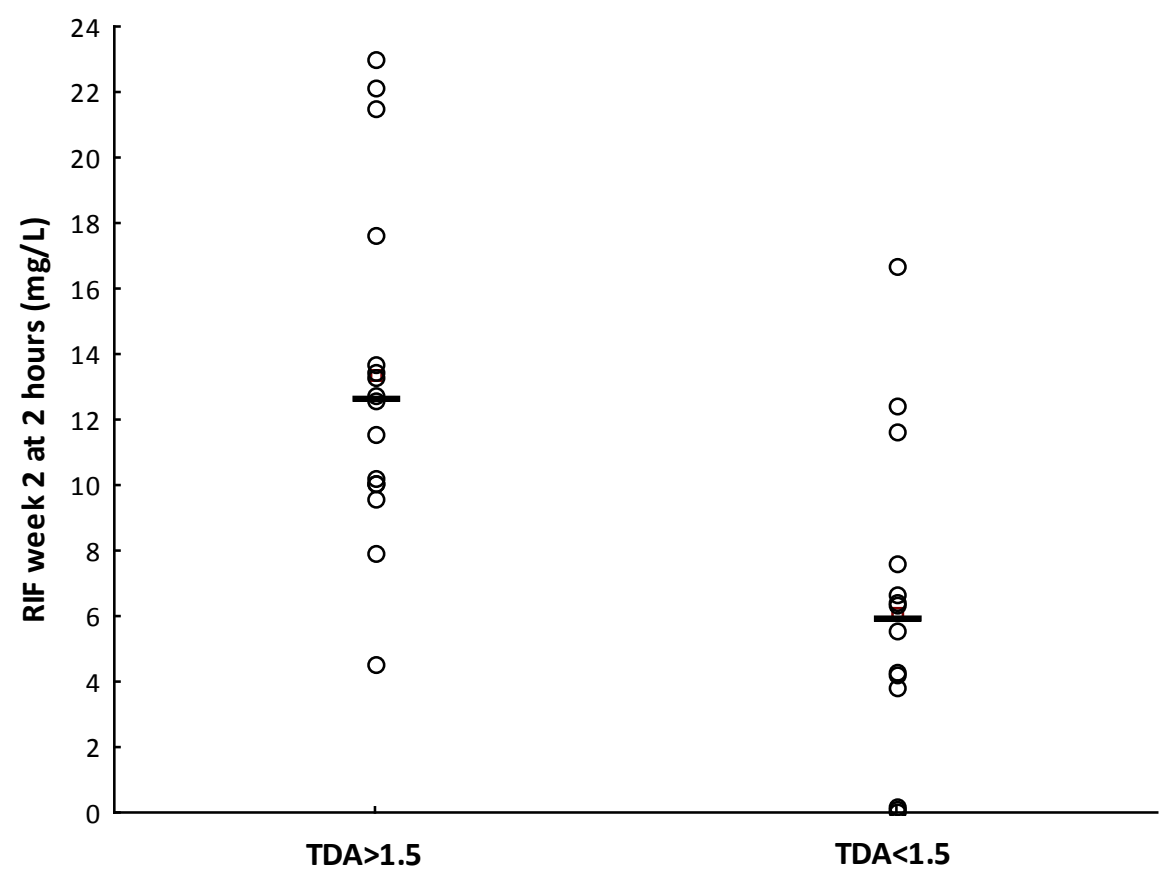

Figure 19. Comparison between plasma rifampicin concentrations and TDA. Vertical bold lines indicate medians. Patients with TDA $<1.5$ exhibited significantly lower rifampicin levels $(p<0.001)$.

RIF: rifampicin, TDA: TB drug activity assay 


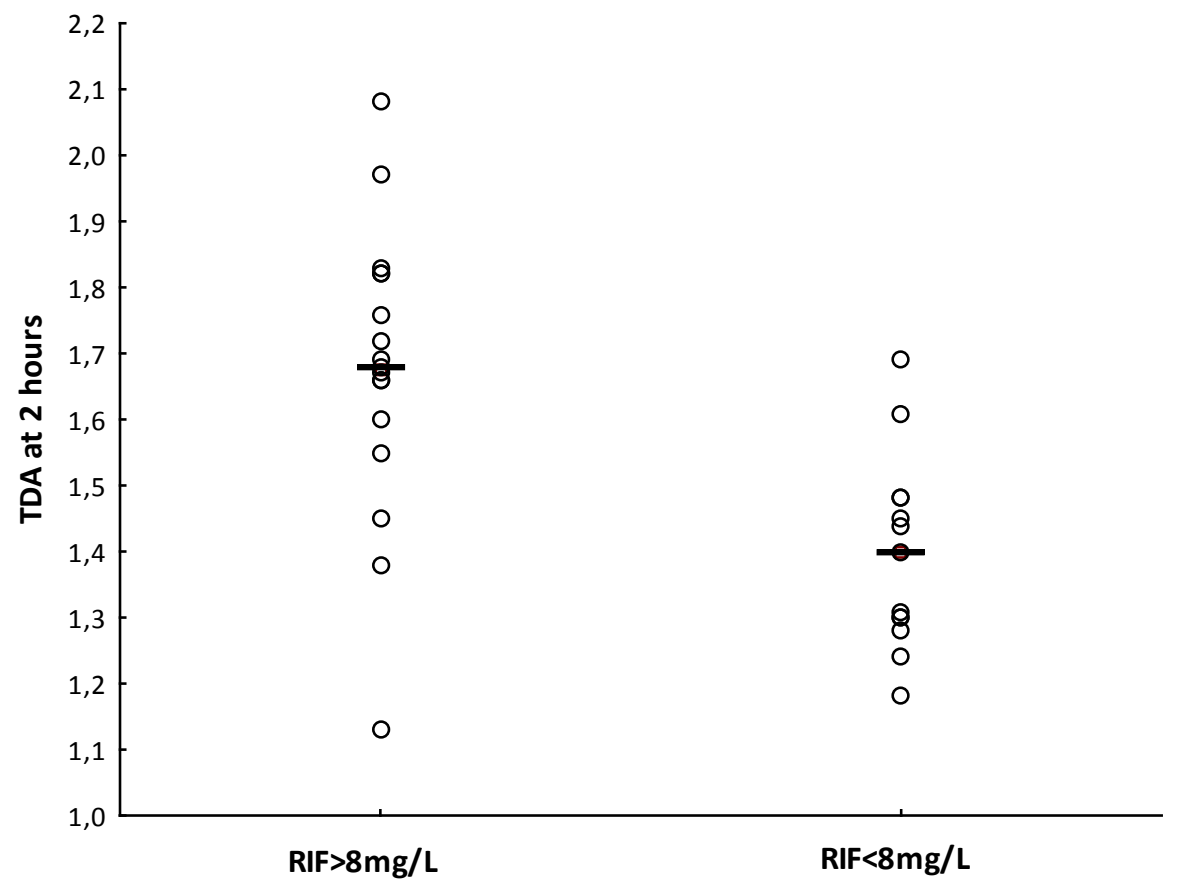

Figure 20. Comparison between TB drug activity in patients with rifampicin concentration above $8 \mathrm{mg} / \mathrm{L}$ and below $8 \mathrm{mg} / \mathrm{L}$. Vertical bold lines indicate medians. ( $p=0.001)$.

RIF: rifampicin, TDA: TB drug activity assay

The precision in the TDA assay in study III expressed as the coefficient of variation $(\mathrm{CV} \%)$ was $9.6 \mathrm{CV} \%$ for intra-assay variability, whereas inter-assay variability was 23.0 $\mathrm{CV} \%$ for the negative and $18.2 \mathrm{CV} \%$ for the positive control. Hence, the intra-assay variability was acceptable, but due to difficulties in precise measurement of the initial bacterial inoculum and the variability of the assay, the inter-assay variability was rather high. However, the clinical impact of this variability may be of less significance as TDM is more based on a semi-quantitative assessment of low, medium or high levels rather than a strict individual level. Nevertheless, it is recommended that a sample taken before dose adjustment should therefore be reanalysed together with the new sample. Moreover, by including the TB patient's individual M. tuberculosis strain instead of a reference strain may provide a more accurate estimate of the $\mathrm{PK} / \mathrm{PD}$ relationship, but this approach may introduce further technical variability. Moreover, by using a reference strain TDM can be performed early in TB treatment for useful dose adjustments.

In conclusion, TDA may be an interesting option to use as a surrogate marker for drug concentration analysis of important first-line drugs especially in resource-limited highburden countries even though TDA requires laboratory facilities for M. tuberculosis culture. 


\section{CONCLUSIONS}

- A mismatch was detected between mutational and phenotypic resistance in breakpoints for ofloxacin and levofloxacin in Middlebrook 7H10 media, which may affect the classification of XDR-TB, treatment of MDR-TB and the evaluation of fluoroquinolones in clinical interventional studies.

- In a low-endemic setting, drug concentrations below reference range for first-line TB drugs were common in general and for rifampicin $(42 \%, 13 / 31)$ in particular.

- An explorative study of first-line PK/PD parameters displayed a wide distribution of the ratios between drug exposures and MICs.

- Rifampicin plasma levels exhibited significantly higher individual fluctuations during the first 12 weeks of TB treatment compared with isoniazid.

- TB patients with plasma levels of rifampicin below reference range $(<8 \mathrm{mg} / \mathrm{L})$ had significantly lower median level of tuberculosis drug activity (TDA) compared with patients with rifampicin concentration exceeding $8 \mathrm{mg} / \mathrm{L}$.

- A novel translational study approach, which investigates the distribution of drug exposure and individual MICs in relation to clinical and microbiological outcome, has shown to be feasible in an MDR-TB setting. 



\section{FUTURE PERSPECTIVES ON INDIVIDUALISED TREATMENT OF TUBERCULOSIS}

Future perspectives on management of TB include both strategies not far from implementation in clinical routine, as well as clinical approaches to combat TB that are visionary and still far from clinical practice. In high-burden and low-resource countries challenges remain in terms of improving living conditions, social structures and health care systems as well as escalating case-finding and contact-tracing. Additionally, drug quality and availability need to be considered and not the least, increasing the access to reliable and in-expensive tools to determine drug resistance to avoid inappropriate treatment, risk of therapy failure and emergence of resistance. The challenge is to bring advances in tools and strategies from high-resource (but low-burden) countries to highendemic areas and patients that need them the most.

\section{Next generation sequencing in clinical routine}

Molecular techniques for DST of M. tuberculosis are rapidly growing and constitute a significant improvement in current management of clinical TB. Although no data on treatment outcome were included, ground breaking work to investigate genotypephenotype correlations of DST has been published by the international consortium CRyPTIC regarding first-line drugs (79). Their aim is to obtain a complete catalogue of resistance-conferring mutations that, ultimately, will phase out routine culture-based assays (224). The ReSeqTB (The Relational Sequencing TB Data Platform) is a global initiative to an open-access catalogue of pooled data on genotypic, phenotypic and related metadata from $M$. tuberculosis strains to facilitate the interpretation of genotypic test results. Efforts are also made to overcome the technical challenges to perform WGS directly from smear/PCR positive specimens, which would significantly reduce time to adjustment of TB treatment according to resistance pattern (100). Moreover, the ongoing re-evaluation of critical concentrations of TB drugs may resolve some of the current systematic differences between genotype and phenotype (59). However, as already told in the mid 60's by Canetti in the Amberson lecture there is a 'need of an agreement on a common protocol of strain testing' (27). More than fifty years later this is still an issue in culture-based DST of mycobacteria and numerous pDST methods are in use world-wide. The lack of a universal standardised method for determination of MIC of first- and second-line TB drugs hampers the interpretation of genotype-phenotype relationships. The process of setting ECOFFs and breakpoints for susceptibility and resistance of old and new TB drugs is dependent on method harmonisation. Furthermore, reliable DST and MIC determination provide more accurate base for defining PK/PD indices important for meaningful TDM. Fortunately, an agreement on a reference method is now a priori for regulatory bodies such as EUCAST responsible for setting pDST breakpoints (225) and has also been acknowledged by the CRyPTIC consortium and the WHO $(59,224)$.

\section{Novel holistic approach in optimising TB treatment}

There are compelling reasons to create new shorter treatment regimens for both drugsusceptible and drug-resistant TB and a key component to reach this goal is most likely 
to accelerate the sterilising drug activity on both extracellular and intracellular tubercle bacilli (226). Clinical trials for evaluation of new TB treatment regimens are expensive and challenging. However, important steps towards fruitful collaboration have been taken such as the establishment of PanACEA (Pan-African Consortium for the Evaluation of Anti-Tuberculosis Antibiotics), a network consisting of European research organisations, 11 sub-Saharan clinical trial sites, and three pharmaceutical companies (227). Several promising studies on treatment optimisation of susceptible TB and MDR-TB are ongoing, and a number of repurposed or new TB-specific drugs are under investigation $(73,228)$. Depending on disease severity and manifestation, treatment length may have to be stratified by group into shorter and longer regimens, which requires guidance by reliable biomarkers predictive of good treatment outcome. Studies on different biomarkers has increased our understanding of the pathogenesis of TB and the continuum between latent and active TB. Nevertheless, a single biomarker that is indicative for treatment response and long-term outcome remains to be identified (229). Optimising the host response, both innate and adaptive immunity, by nutritional supplementation and adjuvant immunotherapy may be potential interventions to prevent TB activation or improve TB cure rates $(8)$.

The pathogenesis of M. tuberculosis in humans is complex and host immunity is a key factor in the defence against both active and latent TB (230). The importance of effective cell-mediated immunity is reflected by the increased risk of TB activation in individuals with HIV as a consequence of the decline in CD4 positive T-lymphocytes $(230,231)$. An increased risk of developing active TB due to promotion of intracellular M. tuberculosis growth is observed during treatment with tumour necrosis factor (TNF)- $\alpha$ inhibitors or other immunosuppressive drugs such as corticosteroids. A number of host immunity checkpoints are present to prevent TB infection and disease. There is a need for increased knowledge of how to strengthen these host barriers to open for immunotherapeutic interventions and monitoring (8). Several host directed therapies have been explored but so far few have reached clinical practice such as the beneficial effects of corticosteroids in treatment of TB meningitis and pericarditis $(8,92)$.

Clearly, the host immune defence against TB is a key player to cure the disease in concert with medical treatment. However, there is an inter-individual variability and ideally the host immune activity should be quantified. Patients with a relatively weak cell-mediated immunity may require optimised TB treatment to compensate for a deficient immune system. Ideally, individualised treatment of TB starts early in the intensive phase with staging of disease severity by radiology and prognostic biomarkers combined with potential measurement of the host's immune system. Based on this information as shown in Figure 21, TB treatment could be optimised and tailored by using TDM where drug doses may be adjusted guided by plasma drug concentration in relation to the susceptibility level of the bacteria. This novel strategy for TB treatment may involve aiming for higher ratios between drug concentration and MIC among patients with more severe disease and weak host immunity. However, PK/PD-targets for TB drugs predictive of favourable treatment outcome remain to be established. 


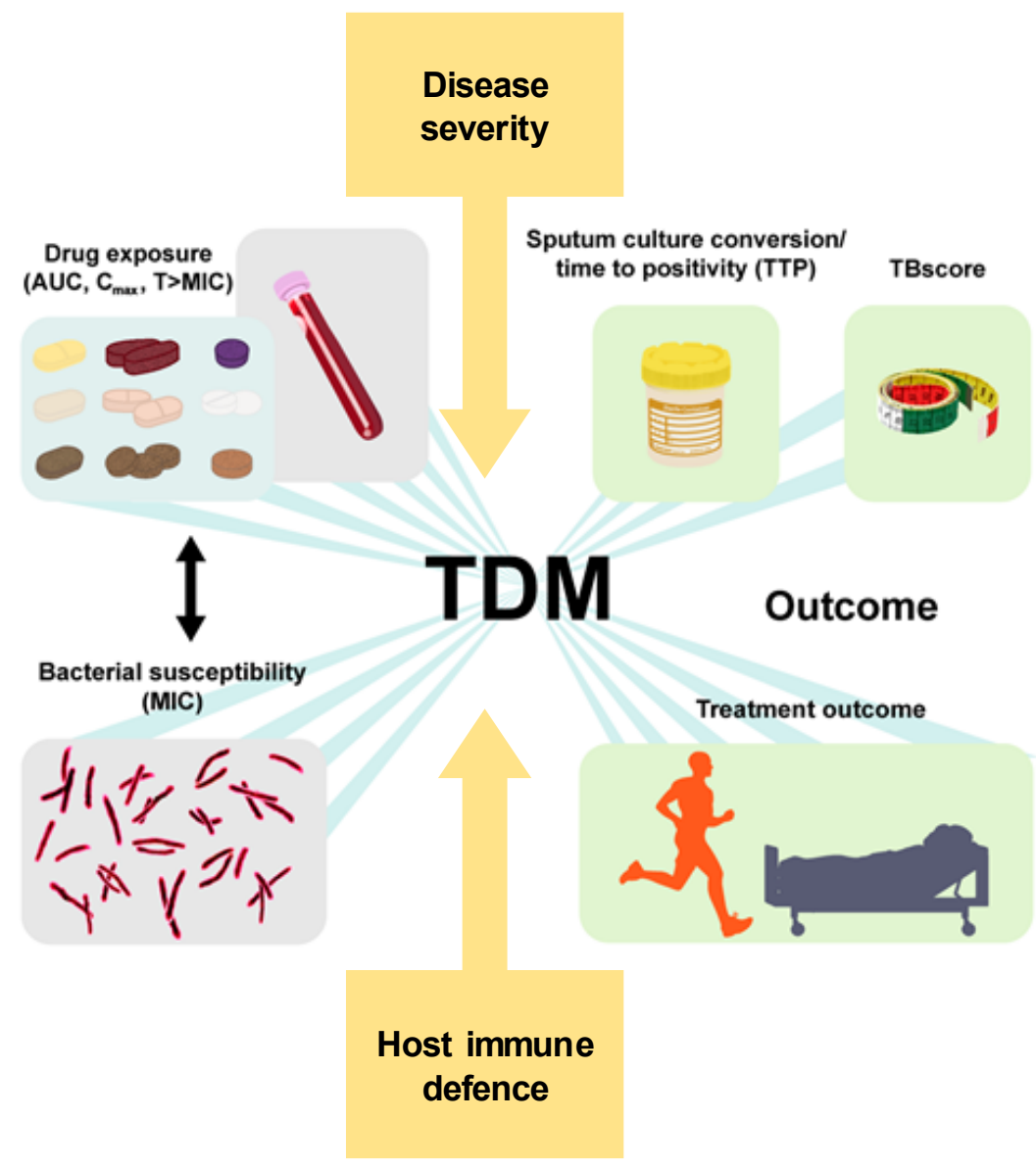

Figure 21. Therapeutic drug monitoring (TDM) in TB with a holistic approach - to correlate drug exposure to the susceptibility level of the bacteria in order to improve treatment outcome and accounting for disease severity and host immune defence.

Blood sampling for measurement of drug concentrations in TDM during TB treatment is traditionally collected after two weeks treatment when steady state of TB drugs is expected. Dose adjustments as early as possible after treatment initiation is to prefer. Model-based TDM for dose guidance enables single PK sampling within the first days of treatment provided simple baseline data of patient's characteristics and dosing are included (e.g. gender, age, time of drug intake and blood sampling).

New techniques for treatment optimisation in low-resource settings

Almost all TB related deaths occur in low-resource settings (3). Unfortunately, new techniques and advances have difficulties to reach high-burden and resource-constrained areas. The burden of drug-resistant TB, mainly concentrated to countries with limited resources, needs to be addressed by a combination of clinical, laboratory and 
programmatic advances. Recently, viewpoints were communicated that highlighted the challenges associated with the use of WGS for diagnosis and management of drugresistant TB in low-resource settings (232). Moreover, monitoring of drug concentrations is rarely feasible in areas with the highest burden of TB and difficult-to-treat cases. Dried blood spot (DBS) sampling for drug concentration analysis may substitute for conventional plasma sampling used for TDM with close access to advanced laboratory facilities (233). DBS offers increased sample stability, require lower blood sample volume and entail less biohazard risk compared with conventional plasma samples. Finger-prick for DBS can be performed in the patient's home, the DBS card may be kept in room temperature and easily transported by mail to a reference laboratory, thus enabling the application of TDM even in remote areas. All main second-line drugs should be included and validated in the methodology for DBS drug analysis.

In conclusion, multiple improvements exist for the clinical management of TB, but the challenge remains to apply cost-benefit analysis and transfer the most appropriate advancements into the areas where they are needed the most. 


\section{ACKNOWLEDGEMENTS}

As a child and young teenager, the thought of becoming a writer crossed my mind as I was encouraged by my teachers who apparently identified something behind my lines of writing. Even though this is a book with my name on the front page, honestly, it is not exactly what I had in mind back then. Most of all, this is not a book I could ever have accomplished on my own. I would like to thank the study participants in Sweden and China for their invaluable contribution and express my deepest gratitude to all of you who have been of help and support during this journey. Thank you ever so much, and especially to:

My outstanding supervisor Thomas Schön whose endless energy, enthusiasm and deep knowledge never have ceased to amaze me. I have still not figured out how you manage to fit all your projects within the $24 \mathrm{~h}$ of the day and yet be able to care about details as well as step into a higher gear when needed. You helped me put perspective in place when it faded and gave me confidence when I needed it. Your scientific mentorship as well as your understanding support when life is challenging, have been invaluable. Thank you !

My colleague, co-worker and co-supervisor (I might have missed some more "co:s") Jakob Paues for making both joint clinical work and research so much more fun, laced with good laughs and interesting intellectual discussions as well as surviving statistics together! I look forward to continuing working with you clinically as well as strengthening the research in our TB group in Östergötland.

I was fortunate to have Judith Bruchfeld as my co-supervisor. Thank you for excellent supervision, in particular regarding all study visits in China and for sharing your extensive clinical experiences in TB, all your wise guidance both in research and in life whenever it was needed. I hope for more clinical and research collaboration as well as uplifting "Judith-guided" visits to TB conferences.

Co-supervisor Maria Lerm for precious advice and opening doors to the pre-clinical field. Your enthusiasm for TB immunology has been inspiring and I hope we will continue exploring translational TB research together. I look forward to TB research projects that did not fit within the framework of this thesis.

My PhD-sister Lina Davies Forsman, in many ways my closest companion during this $\mathrm{PhD}$ project, for all the mutual help and support we could offer each other during the writing of our theses. Thanks for great friendship and memorable times as "Lina och Tina i Kina" and time spent together with our families. Johanna Kuhlin for joining the project in China and for selfless sharing of her work.

Michaela Nordvall Jonsson for your patience and willingness to share your skills on several occasions in the TB-laboratory in Linköping. Jim Werngren, Mikael Mansjö and Maria Wijkander for valuable visits and workshops at the Public Health Agency, and Erja Chryssantou and Linnea Ek Blom at the TB-laboratory in Solna. Anders Helldén and Björn Carlsson at the department of Clinical Pharmacology in Linköping for collaborations and valuable support with pharmacokinetics and fruitful projects with 
Ulrika Simonsson and Robin Svensson at Uppsala University providing in-depth knowledge in pharmacometrics.

All co-authors for excellent work with the papers constituting my thesis as well as Hannah Schulman for collaboration in the most recent paper - I miss you in Östergötland!

All members of the joint project in China, especially professor Xu Biao at the Fudan University, Shanghai, and Principal Investigator Sven Hoffner at the Public Health Agency in Sweden, and the dedicated hard-working team of Yi Hu, Xubin Zheng, and Dr Zheng.

Former research nurse Lotta Lindvall for outstanding practical help in the clinical study at the sites in Östergötland - it was a true pleasure working with you and you are very much missed at the clinic. TB-nurses and staff at the out-patient clinics in Linköping and Norrköping for excellent work with our study participants. Study nurses Jan Bellbrant and Ingrid Andrén for similar invaluable work at the study site in Solna.

Special thanks and gratitude to all my colleagues at the department of Infectious Diseases and the head of the department Carola Skogwik, for supporting my research and maintaining the good working climate.

Professor emeritus Pia Forsberg who inspired me not to abandon the research pathway, and former colleagues and heads at the department of Infectious Diseases, Rolf Maller and Aril Frydén for being such good role models.

My colleagues, Åse Östholm Balkhed (true WF-friend) and Johanna Sjöwall for "fikas", splitting on-call shifts and precious advice during the writing. Lena Änghagen and Kristina Cardell, for friendship and much appreciated support when at times life brings challenges - thanks for being there!

My former business partner, inspiring golf companion and dear friend, Emma Stockman for all the good talks and reminding me of the valuable things in life. My dear friend Malin Lyreborn for invigorating ladies' night dinners with long talks.

My close and precious friend for twenty years, Sofia Sederholm Lawesson, thank you for always being there in ups and downs and for all valuable inputs!

My late beloved parents, Febe and Bo, for endless love and support, without you this would not have been accomplished - I miss you so much. My dear brother Peter for always being there for me. Ros-Mari and Bo, my parents-in-law, your care and support have meant so much to me. My beloved children and greatest gift in life, Lucas and Marcus, you make all the difference! Last but not least, my beloved husband and best friend Hans, for all encouragement, love and tremendous support - thanks for sharing life and making it such an adventure! 


\section{REFERENCES}

1. Schaaf HS, Zumla A. Tuberculosis; A Comprehensive Clinical Reference. London UK: WB Saunders Elsevier; 2009.

2. Iseman MD. A clinician's guide to tuberculosis. Philadelphia USA: Lippincott Williams \& Wilkins; 2000.

3. World Health Organization. Global tuberculosis report 2018. Available from: https://www.who.int/tb/publications/global report/en/.

4. Public Health Agency of Sweden. Tuberkulos 2018 [cited 2019 Apr 03] Available from: https://www.folkhalsomyndigheten.se/folkhalsorapportering-statistik/statistikdatabaser-ochvisualisering/sjukdomsstatistik/tuberkulos/.

5. Bloemberg GV, Keller PM, Stucki D, Trauner A, Borrell S, Latshang T, et al. Acquired Resistance to Bedaquiline and Delamanid in Therapy for Tuberculosis. N Engl J Med. 2015;373(20):1986-8.

6. Ducati RG, Ruffino-Netto A, Basso LA, Santos DS. The resumption of consumption -- a review on tuberculosis. Mem Inst Oswaldo Cruz. 2006;101(7):697-714.

7. Guenin-Mace L, Simeone R, Demangel C. Lipids of pathogenic Mycobacteria: contributions to virulence and host immune suppression. Transbound Emerg Dis. 2009;56(6-7):255-68.

8. Schon T, Lerm M, Stendahl O. Shortening the 'short-course' therapy-insights into host immunity may contribute to new treatment strategies for tuberculosis. J Intern Med. 2013;273(4):368-82.

9. Pai M, Behr MA, Dowdy D, Dheda K, Divangahi M, Boehme CC, et al. Tuberculosis. Nat Rev Dis Primers. 2016;2:16076.

10. Erkens CG, Kamphorst M, Abubakar I, Bothamley GH, Chemtob D, Haas W, et al. Tuberculosis contact investigation in low prevalence countries: a European consensus. Eur Respir J. 2010;36(4):925-49.

11. World Health Organization. Latent TB Infection : Updated and consolidated guidelines for programmatic management. 2018. Available from:

https://www.who.int/tb/publications/2018/latent-tuberculosis-infection/en/.

12. Mitchison DA. The action of antituberculosis drugs in short-course chemotherapy. Tubercle. 1985;66(3):219-25.

13. Gumbo T, Dona CS, Meek C, Leff R. Pharmacokinetics-pharmacodynamics of pyrazinamide in a novel in vitro model of tuberculosis for sterilizing effect: a paradigm for faster assessment of new antituberculosis drugs. Antimicrob Agents Chemother. 2009;53(8):3197-204.

14. Hu Y, Liu A, Ortega-Muro F, Alameda-Martin L, Mitchison D, Coates A. High-dose rifampicin kills persisters, shortens treatment duration, and reduces relapse rate in vitro and in vivo. Front Microbiol. 2015;6:641.

15. World Health Organization. Treatment of Tuberculosis - guidelines for national programmes, 4th edition, 2010. Available from: https://www.who.int/tb/publications/9789241547833/en/.

16. World Health Organization. Definitions and reporting framework for tuberculosis 2013 revision, updated December 2014, Geneva: World Health Organization Available from: https://www.who.int/tb/publications/definitions/en/. 
17. Babafemi EO, Cherian BP, Banting L, Mills GA, Ngianga K, 2nd. Effectiveness of real-time polymerase chain reaction assay for the detection of Mycobacterium tuberculosis in pathological samples: a systematic review and meta-analysis. Syst Rev. 2017;6(1):215.

18. Tortoli E, Urbano P, Marcelli F, Simonetti TM, Cirillo DM. Is real-time PCR better than conventional PCR for Mycobacterium tuberculosis complex detection in clinical samples? I Clin Microbiol. 2012;50(8):2810-3.

19. World Health Organization. Compendium of WHO guidelines and associated standards, second edition. 2018. Available from: https://www.who.int/tb/publications/Compendium WHO guidelines TB 2017/en/.

20. Dorman SE, Schumacher SG, Alland D, Nabeta P, Armstrong DT, King B, et al. Xpert MTB/RIF Ultra for detection of Mycobacterium tuberculosis and rifampicin resistance: a prospective multicentre diagnostic accuracy study. Lancet Infect Dis. 2018;18(1):76-84.

21. World Health Organization. Companion handbook to the WHO guidelines for the programmatic management of drug-resistant tuberculosis. 2014. Available from: http://www.who.int/iris/handle/10665/130918.

22. Zhang $\mathrm{Y}$, Yew WW. Mechanisms of drug resistance in Mycobacterium tuberculosis. Int J Tuberc Lung Dis. 2009;13(11):1320-30.

23. Cohen KA, Bishai WR, Pym AS. Molecular Basis of Drug Resistance in Mycobacterium tuberculosis. Microbiol Spectr. 2014;2(3).

24. Dheda K, Gumbo T, Maartens G, Dooley KE, McNerney R, Murray M, et al. The epidemiology, pathogenesis, transmission, diagnosis, and management of multidrug-resistant, extensively drug-resistant, and incurable tuberculosis. Lancet Respir Med. 2017.

25. Andries K, Villellas C, Coeck N, Thys K, Gevers T, Vranckx L, et al. Acquired resistance of Mycobacterium tuberculosis to bedaquiline. PLoS One. 2014;9(7):e102135.

26. Srivastava S, Musuka S, Sherman C, Meek C, Leff R, Gumbo T. Efflux-pump-derived multiple drug resistance to ethambutol monotherapy in Mycobacterium tuberculosis and the pharmacokinetics and pharmacodynamics of ethambutol. J Infect Dis. 2010;201(8):1225-31.

27. Canetti G. Present aspects of bacterial resistance in tuberculosis. Am Rev Respir Dis. 1965;92(5):687-703.

28. Selkon JB, Devadatta S, Kulkarni KG, Mitchison DA, Narayana AS, Nair CN, et al. The emergence of isoniazid-resistant cultures in patients with pulmonary tuberculosis during treatment with isoniazid alone or isoniazid plus PAS. Bull World Health Organ. 1964;31:273-94.

29. Zumla A, Nahid P, Cole ST. Advances in the development of new tuberculosis drugs and treatment regimens. Nat Rev Drug Discov. 2013;12(5):388-404.

30. World Health Organization. Guidelines for treatment of drug-susceptible tuberculosis and patient care (2017 update). Available from:

https://www.who.int/tb/publications/2017/dstb_guidance_2017/en/.

31. Zhang $Y$, Yew WW. Mechanisms of drug resistance in Mycobacterium tuberculosis: update 2015. Int J Tuberc Lung Dis. 2015;19(11):1276-89.

32. Wehrli W, Staehelin M. Actions of the rifamycins. Bacteriol Rev. 1971;35(3):290-309.

33. Grayson ML, Crowe S, McCarthy JS, Mills J, Mouton JW, Norrby SR, et al. Kucers' The Use of Antibiotics: A Clinical Review of Antibacterial, Antifungal, Antiparasitic, and Antiviral Drugs, 6th ed. London UK: Hodder Arnold an Hachette UK Company; 2010. 
34. Zhang $\mathrm{Y}$, Shi W, Zhang W, Mitchison D. Mechanisms of Pyrazinamide Action and Resistance. Microbiol Spectr. 2014;2(4):Mgm2-0023-2013.

35. Fox W, Ellard GA, Mitchison DA. Studies on the treatment of tuberculosis undertaken by the British Medical Research Council tuberculosis units, 1946-1986, with relevant subsequent publications. Int J Tuberc Lung Dis. 1999;3(10 Suppl 2):S231-79.

36. Mitchison DA. Role of individual drugs in the chemotherapy of tuberculosis. Int J Tuberc Lung Dis. 2000;4(9):796-806.

37. Jindani A, Dore CJ, Mitchison DA. Bactericidal and sterilizing activities of antituberculosis drugs during the first 14 days. Am J Respir Crit Care Med. 2003;167(10):1348-54.

38. Jindani A, Aber VR, Edwards EA, Mitchison DA. The early bactericidal activity of drugs in patients with pulmonary tuberculosis. Am Rev Respir Dis. 1980;121(6):939-49.

39. Kempker RR, Heinrichs MT, Nikolaishvili K, Sabulua I, Bablishvili N, Gogishvili S, et al. Lung Tissue Concentrations of Pyrazinamide among Patients with Drug-Resistant Pulmonary Tuberculosis. Antimicrob Agents Chemother. 2017;61(6).

40. Srivastava S, Pasipanodya JG, Gumbo T. pH Conditions under Which Pyrazinamide Works in Humans. Antimicrob Agents Chemother. 2017;61(9).

41. Kempker RR, Peloquin CA, Blumberg HM, Vashakidze S. Reply to Srivastava et al., "pH Conditions under Which Pyrazinamide Works in Humans". Antimicrob Agents Chemother. 2017;61(9).

42. Reynolds J, Heysell SK. Understanding pharmacokinetics to improve tuberculosis treatment outcome. Expert Opin Drug Metab Toxicol. 2014;10(6):813-23.

43. Weiner M, Burman W, Vernon A, Benator D, Peloquin CA, Khan A, et al. Low isoniazid concentrations and outcome of tuberculosis treatment with once-weekly isoniazid and rifapentine. Am J Respir Crit Care Med. 2003;167(10):1341-7.

44. Pasipanodya JG, Srivastava S, Gumbo T. Meta-analysis of clinical studies supports the pharmacokinetic variability hypothesis for acquired drug resistance and failure of antituberculosis therapy. Clin Infect Dis. 2012;55(2):169-77.

45. Gegia M, Winters N, Benedetti A, van Soolingen D, Menzies D. Treatment of isoniazid-resistant tuberculosis with first-line drugs: a systematic review and meta-analysis. Lancet Infect Dis. 2017;17(2):223-34.

46. Weis SE, Slocum PC, Blais FX, King B, Nunn M, Matney GB, et al. The effect of directly observed therapy on the rates of drug resistance and relapse in tuberculosis. N Engl J Med. 1994;330(17):1179-84.

47. Rusen ID, Ait-Khaled N, Alarcon E, Billo N, Bissell K, Boillot F, et al. Cochrane systematic review of directly observed therapy for treating tuberculosis: good analysis of the wrong outcome. Int J Tuberc Lung Dis. 2007;11(2):120-1.

48. Karumbi J, Garner P. Directly observed therapy for treating tuberculosis. Cochrane Database Syst Rev. 2015(5):Cd003343.

49. World Health Organization. Handbook for the use of digital technologies to support tuberculosis medication adherence. 2017. Available from:

https://www.who.int/tb/publications/2018/TB medication adherence handbook 2018/en/ 
50. Imperial MZ, Nahid P, Phillips PPJ, Davies GR, Fielding K, Hanna D, et al. A patient-level pooled analysis of treatment-shortening regimens for drug-susceptible pulmonary tuberculosis. Nat Med. 2018;24(11):1708-15.

51. Jayaram R, Gaonkar S, Kaur P, Suresh BL, Mahesh BN, Jayashree R, et al. Pharmacokineticspharmacodynamics of rifampin in an aerosol infection model of tuberculosis. Antimicrob Agents Chemother. 2003;47(7):2118-24.

52. Stott KE, Pertinez H, Sturkenboom MGG, Boeree MJ, Aarnoutse R, Ramachandran G, et al. Pharmacokinetics of rifampicin in adult TB patients and healthy volunteers: a systematic review and meta-analysis. J Antimicrob Chemother. 2018;73(9):2305-13.

53. Alsultan A, Peloquin CA. Therapeutic drug monitoring in the treatment of tuberculosis: an update. Drugs. 2014;74(8):839-54.

54. Aarnoutse RE, Kibiki GS, Reither K, Semvua HH, Haraka F, Mtabho CM, et al. Pharmacokinetics, Tolerability, and Bacteriological Response of Rifampin Administered at 600, 900, and 1,200 Milligrams Daily in Patients with Pulmonary Tuberculosis. Antimicrob Agents Chemother. 2017;61(11).

55. Ruslami R, Nijland HM, Alisjahbana B, Parwati I, van Crevel R, Aarnoutse RE. Pharmacokinetics and tolerability of a higher rifampin dose versus the standard dose in pulmonary tuberculosis patients. Antimicrob Agents Chemother. 2007;51(7):2546-51.

56. Boeree MJ, Diacon AH, Dawson R, Narunsky K, du Bois J, Venter A, et al. A dose-ranging trial to optimize the dose of rifampin in the treatment of tuberculosis. Am J Respir Crit Care Med. 2015;191(9):1058-65.

57. Velasquez GE, Brooks MB, Coit JM, Pertinez H, Vargas Vasquez D, Sanchez Garavito E, et al. Efficacy and Safety of High-Dose Rifampin in Pulmonary Tuberculosis. A Randomized Controlled Trial. Am J Respir Crit Care Med. 2018;198(5):657-66.

58. Boeree MJ, Heinrich N, Aarnoutse R, Diacon AH, Dawson R, Rehal S, et al. High-dose rifampicin, moxifloxacin, and SQ109 for treating tuberculosis: a multi-arm, multi-stage randomised controlled trial. Lancet Infect Dis. 2017;17(1):39-49.

59. World Health Organization. Technical report on critical concentrations for drug susceptibility testing of medicines used in the treatment of drug-resistant tuberculosis; World Health Organization; 2018. Available from: http://apps.who.int/iris/handle/10665/260470.

60. World Health Organization. Rapid Communication: Key changes to treatment of multidrugand rifampicin-resistant tuberculosis (MDR/RR-TB). Updated August 2018. Available from: http://www.who.int/tb/publications/2018/WHO RapidCommunicationMDRTB.pdf.

61. Lange C, Chesov D, Furin J, Udwadia Z, Dheda K. Revising the definition of extensively drugresistant tuberculosis. Lancet Respir Med. 2018.

62. World Health Organization. WHO treatment guidelines for drug-resistant tuberculosis (2016 update). Available from: https://www.who.int/tb/areas-of-work/drug-resistanttb/treatment/resources/en/.

63. Davies Forsman L, Jonsson J, Wagrell C, Werngren J, Mansjo M, Wijkander M, et al. Minimum inhibitory concentrations of fluoroquinolones and pyrazinamide susceptibility correlate to clinical improvement in MDR-TB patients - a nationwide Swedish cohort study over two decades. Clin Infect Dis. 2018.

64. Shandil RK, Jayaram R, Kaur P, Gaonkar S, Suresh BL, Mahesh BN, et al. Moxifloxacin, ofloxacin, sparfloxacin, and ciprofloxacin against Mycobacterium tuberculosis: evaluation of in vitro and 
pharmacodynamic indices that best predict in vivo efficacy. Antimicrob Agents Chemother. 2007;51(2):576-82.

65. Deshpande D, Pasipanodya JG, Mpagama SG, Bendet P, Srivastava S, Koeuth T, et al. Levofloxacin Pharmacokinetics/Pharmacodynamics, Dosing, Susceptibility Breakpoints, and Artificial Intelligence in the Treatment of Multidrug-resistant Tuberculosis. Clin Infect Dis. 2018;67(suppl_3):S293-s302.

66. Ahmad N, Ahuja SD, Akkerman OW, Alffenaar JC, Anderson LF, Baghaei P, et al. Treatment correlates of successful outcomes in pulmonary multidrug-resistant tuberculosis: an individual patient data meta-analysis. Lancet. 2018;392(10150):821-34.

67. World Health Organization. WHO treatment guidelines for isoniazid-resistant tuberculosis. 2018. Available from: https://www.who.int/tb/publications/2018/WHO guidelines isoniazid resistant TB/en/.

68. Nijland HM, Ruslami R, Suroto AJ, Burger DM, Alisjahbana B, van Crevel R, et al. Rifampicin reduces plasma concentrations of moxifloxacin in patients with tuberculosis. Clin Infect Dis. 2007;45(8):1001-7.

69. Pranger AD, van der Werf TS, Kosterink JGW, Alffenaar JWC. The Role of Fluoroquinolones in the Treatment of Tuberculosis in 2019. Drugs. 2019.

70. World Health Organization. Technical report on the pharmacokinetics and pharmacodynamics (PK/PD) of medicines used in the treatment of drug-resistant tuberculosis; World Health Organization; 2018. Available from: http://www.who.int/iris/handle/10665/260440.

71. Nunn AJ, Phillips PPJ, Meredith SK, Chiang CY, Conradie F, Dalai D, et al. A Trial of a Shorter Regimen for Rifampin-Resistant Tuberculosis. N Engl J Med. 2019.

72. Lange C, Duarte R, Frechet-Jachym M, Guenther G, Guglielmetti L, Olaru ID, et al. Limited Benefit of the New Shorter Multidrug-Resistant Tuberculosis Regimen in Europe. Am J Respir Crit Care Med. 2016;194(8):1029-31.

73. Lange C, Chesov D, Heyckendorf J, Leung CC, Udwadia Z, Dheda K. Drug-resistant tuberculosis: An update on disease burden, diagnosis and treatment. Respirology. 2018;23(7):656-73.

74. Canetti G, Froman S, Grosset J, Hauduroy P, Langerova M, Mahler HT, et al. Mycobacteria: Laboratory Methods for Testing Drug Sensitivity and Resistance. Bull World Health Organ. 1963;29:565-78.

75. Schon T, Miotto P, Koser CU, Viveiros M, Bottger E, Cambau E. Mycobacterium tuberculosis drug-resistance testing: challenges, recent developments and perspectives. Clin Microbiol Infect. 2017;23(3):154-60.

76. Bastos ML, Hussain H, Weyer K, Garcia-Garcia L, Leimane V, Leung CC, et al. Treatment outcomes of patients with multidrug-resistant and extensively drug-resistant tuberculosis according to drug susceptibility testing to first- and second-line drugs: an individual patient data meta-analysis. Clin Infect Dis. 2014;59(10):1364-74.

77. Kim SJ. Drug-susceptibility testing in tuberculosis: methods and reliability of results. Eur Respir J. 2005;25(3):564-9.

78. Kahlmeter G. Defining antibiotic resistance-towards international harmonization. Ups J Med Sci. 2014;119(2):78-86.

79. Allix-Beguec C, Arandjelovic I, Bi L, Beckert P, Bonnet M, Bradley P, et al. Prediction of Susceptibility to First-Line Tuberculosis Drugs by DNA Sequencing. N Engl J Med. 2018;379(15):1403-15. 
80. StopTBpartnership. Standard Operating Procedure Drug Susceptibility Testing, Proportion Method. Available from: http://www.stoptb.org/wg/gli/sops.asp.

81. Canetti G, Fox W, Khomenko A, Mahler HT, Menon NK, Mitchison DA, et al. Advances in techniques of testing mycobacterial drug sensitivity, and the use of sensitivity tests in tuberculosis control programmes. Bull World Health Organ. 1969;41(1):21-43.

82. Cambau E, Rüsch-Gerdes S. First and second line susceptibility testing for mycobacterium tuberculosis complex. In: Handbook on TB laboratory diagnostic methods for the European Union European Center for disease prevention and control (ECDC) ed. Stockholm: ECDC; 2016. p. $72 \mathrm{e} 82$.

83. Clinical and Laboratory Standards Institute (CLSI). Susceptibility Testing of Mycobacteria, Nocardia spp., and Other Aerobic Actinomycetes, 3rd Edition. Nov 2018. Available from: https://clsi.org/standards/products/microbiology/documents/m24/.

84. Mouton JW, Meletiadis J, Voss A, Turnidge J. Variation of MIC measurements: the contribution of strain and laboratory variability to measurement precision. J Antimicrob Chemother. 2018;73(9):2374-9.

85. Heysell SK, Pholwat S, Mpagama SG, Pazia SJ, Kumburu H, Ndusilo N, et al. Sensititre MycoTB plate compared to Bactec MGIT 960 for first- and second-line antituberculosis drug susceptibility testing in Tanzania: a call to operationalize MICs. Antimicrob Agents Chemother. 2015;59(11):7104-8.

86. Angeby K, Jureen $P$, Kahlmeter G, Hoffner SE, Schon T. Challenging a dogma: antimicrobial susceptibility testing breakpoints for Mycobacterium tuberculosis. Bull World Health Organ. 2012;90(9):693-8.

87. European Committee on Antimicrobial Susceptibility Testing. MIC distributions and epidemiological cut-off value (ECOFF) setting, EUCAST SOP 10.0, 2017. Available from: http://www.eucast.org.

88. European Committee on Antimicrobial Susceptibility Testing. Implications of breakpoints splitting the wild type and/or resistant populations. EUCAST, 2016. Available from: http://www.eucast.org.

89. Kahlmeter G. The 2014 Garrod Lecture: EUCAST - are we heading towards international agreement? J Antimicrob Chemother. 2015;70(9):2427-39.

90. European Committee on Antimicrobial Susceptibility Testing. The implementation of the new definitions of S, I and R. EUCAST, 2019. The presentation in its original form can be found at www.eucast.org.

91. Kaniga K, Cirillo DM, Hoffner S, Ismail NA, Kaur D, Lounis N, et al. A Multilaboratory, Multicountry Study To Determine MIC Quality Control Ranges for Phenotypic Drug Susceptibility Testing of Selected First-Line Antituberculosis Drugs, Second-Line Injectables, Fluoroquinolones, Clofazimine, and Linezolid. J Clin Microbiol. 2016;54(12):2963-8.

92. Lange $\mathrm{C}$, Alghamdi WA, Al-Shaer MH, Brighenti S, Diacon AH, DiNardo AR, et al. Perspectives for personalized therapy for patients with multidrug-resistant tuberculosis. J Intern Med. 2018.

93. Miotto P, Tessema B, Tagliani E, Chindelevitch L, Starks AM, Emerson C, et al. A standardised method for interpreting the association between mutations and phenotypic drug resistance in Mycobacterium tuberculosis. Eur Respir J. 2017;50(6).

94. World Health Organization. The use of molecular line probe assays for the detection of resistance to second-line anti-tuberculosis drugs. Policy guidance. World Health Organization 
2016. Available from: https://www.who.int/tb/publications/policy-guidance-molecularline/en/.

95. Theron G, Peter J, Richardson M, Warren R, Dheda K, Steingart KR. GenoType((R)) MTBDRsI assay for resistance to second-line anti-tuberculosis drugs. Cochrane Database Syst Rev. 2016;9:Cd010705.

96. Hillemann D, Mitchell S, Drobniewski F. Identification of Mycobacterium tuberculosis and drug resistance in cultures and sputum using molecular assays and immunoassays. In: Handbook on TB laboratory diagnostic methods for the European Union European Center for disease prevention and control (ECDC) ed. Stockholm: ECDC; 2016. p. 54e71.

97. World Health Organization. WHO Meeting Report of a Technical Expert Consultation: Noninferiority analysis of Xpert MTB/RIF Ultra compared to Xpert MTB/RIF. 2017. Available from: https://www.who.int/tb/publications/2017/XpertUltra/en/.

98. Pankhurst L, Del Ojo Elias C, Votintseva AA, Walker TM, Cole K, Davies J, et al. Rapid, comprehensive, and affordable mycobacterial diagnosis with whole-genome sequencing: a prospective study. Lancet Respir Med. 2016;4(1):49-58.

99. Colijn C, Cohen T. Whole-genome sequencing of Mycobacterium tuberculosis for rapid diagnostics and beyond. Lancet Respir Med. 2016;4(1):6-8.

100. Brown AC, Bryant JM, Einer-Jensen K, Holdstock J, Houniet DT, Chan JZ, et al. Rapid WholeGenome Sequencing of Mycobacterium tuberculosis Isolates Directly from Clinical Samples. J Clin Microbiol. 2015;53(7):2230-7.

101. Dominguez J, Boettger EC, Cirillo D, Cobelens F, Eisenach KD, Gagneux S, et al. Clinical implications of molecular drug resistance testing for Mycobacterium tuberculosis: a TBNET/RESIST-TB consensus statement. Int J Tuberc Lung Dis. 2016;20(1):24-42.

102. Avalos E, Catanzaro D, Catanzaro A, Ganiats T, Brodine S, Alcaraz J, et al. Frequency and geographic distribution of gyrA and gyrB mutations associated with fluoroquinolone resistance in clinical Mycobacterium tuberculosis isolates: a systematic review. PLoS One. 2015;10(3):e0120470.

103. Schon T, Jureen P, Giske CG, Chryssanthou E, Sturegard E, Werngren J, et al. Evaluation of wildtype MIC distributions as a tool for determination of clinical breakpoints for Mycobacterium tuberculosis. J Antimicrob Chemother. 2009;64(4):786-93.

104. Walker TM, Kohl TA, Omar SV, Hedge J, Del Ojo Elias C, Bradley P, et al. Whole-genome sequencing for prediction of Mycobacterium tuberculosis drug susceptibility and resistance: a retrospective cohort study. Lancet Infect Dis. 2015;15(10):1193-202.

105. Wallis RS, Pai M, Menzies D, Doherty TM, Walzl G, Perkins MD, et al. Biomarkers and diagnostics for tuberculosis: progress, needs, and translation into practice. Lancet. 2010;375(9729):1920-37.

106. Phillips PP, Fielding K, Nunn AJ. An evaluation of culture results during treatment for tuberculosis as surrogate endpoints for treatment failure and relapse. PLoS One. 2013;8(5):e63840.

107. Horne DJ, Royce SE, Gooze L, Narita M, Hopewell PC, Nahid P, et al. Sputum monitoring during tuberculosis treatment for predicting outcome: systematic review and meta-analysis. Lancet Infect Dis. 2010;10(6):387-94.

108. Nahid P, Dorman SE, Alipanah N, Barry PM, Brozek JL, Cattamanchi A, et al. Official American Thoracic Society/Centers for Disease Control and Prevention/Infectious Diseases Society of 
America Clinical Practice Guidelines: Treatment of Drug-Susceptible Tuberculosis. Clin Infect Dis. 2016;63(7):e147-e95.

109. Kurbatova EV, Cegielski JP, Lienhardt C, Akksilp R, Bayona J, Becerra MC, et al. Sputum culture conversion as a prognostic marker for end-of-treatment outcome in patients with multidrugresistant tuberculosis: a secondary analysis of data from two observational cohort studies. Lancet Respir Med. 2015;3(3):201-9.

110. Diacon AH, Donald PR. The early bactericidal activity of antituberculosis drugs. Expert Rev Anti Infect Ther. 2014;12(2):223-37.

111. Diacon AH, Dawson R, von Groote-Bidlingmaier F, Symons G, Venter A, Donald PR, et al. Bactericidal activity of pyrazinamide and clofazimine alone and in combinations with pretomanid and bedaquiline. Am J Respir Crit Care Med. 2015;191(8):943-53.

112. Diacon AH, van der Merwe L, Demers AM, von Groote-Bidlingmaier F, Venter A, Donald PR. Time to positivity in liquid culture predicts colony forming unit counts of Mycobacterium tuberculosis in sputum specimens. Tuberculosis (Edinb). 2014;94(2):148-51.

113. Diacon AH, Maritz JS, Venter A, van Helden PD, Dawson R, Donald PR. Time to liquid culture positivity can substitute for colony counting on agar plates in early bactericidal activity studies of antituberculosis agents. Clin Microbiol Infect. 2012;18(7):711-7.

114. Ralph AP, Ardian M, Wiguna A, Maguire GP, Becker NG, Drogumuller G, et al. A simple, valid, numerical score for grading chest $x$-ray severity in adult smear-positive pulmonary tuberculosis. Thorax. 2010;65(10):863-9.

115. Palaci M, Dietze R, Hadad DJ, Ribeiro FK, Peres RL, Vinhas SA, et al. Cavitary disease and quantitative sputum bacillary load in cases of pulmonary tuberculosis. J Clin Microbiol. 2007;45(12):4064-6.

116. Romanowski K, Balshaw RF, Benedetti A, Campbell JR, Menzies D, Ahmad Khan F, et al. Predicting tuberculosis relapse in patients treated with the standard 6-month regimen: an individual patient data meta-analysis. Thorax. 2018

117. Wejse C, Gustafson P, Nielsen J, Gomes VF, Aaby P, Andersen PL, et al. TBscore: Signs and symptoms from tuberculosis patients in a low-resource setting have predictive value and may be used to assess clinical course. Scand J Infect Dis. 2008;40(2):111-20.

118. Rudolf F, Lemvik G, Abate E, Verkuilen J, Schon T, Gomes VF, et al. TBscore II: refining and validating a simple clinical score for treatment monitoring of patients with pulmonary tuberculosis. Scand J Infect Dis. 2013;45(11):825-36.

119. Peloquin CA. Therapeutic drug monitoring of the antimycobacterial drugs. Clin Lab Med. 1996;16(3):717-29.

120. Rowland M, Tozer TN. Clinical pharmacokinetics and pharmacodynamics; Concepts and applications, 4th ed. Philadelphia USA: Lippincott Williams and Wilkins; 2011.

121. Zuur MA, Bolhuis MS, Anthony R, den Hertog A, van der Laan T, Wilffert B, et al. Current status and opportunities for therapeutic drug monitoring in the treatment of tuberculosis. Expert Opin Drug Metab Toxicol. 2016;12(5):509-21.

122. Gabrielsson J, Weiner D. Non-compartmental analysis. Methods Mol Biol. 2012;929:377-89.

123. Mouton JW, Brown DF, Apfalter P, Canton R, Giske CG, Ivanova M, et al. The role of pharmacokinetics/pharmacodynamics in setting clinical MIC breakpoints: the EUCAST approach. Clin Microbiol Infect. 2012;18(3):E37-45. 
124. Ambrose PG, Bhavnani SM, Rubino CM, Louie A, Gumbo T, Forrest A, et al. Pharmacokineticspharmacodynamics of antimicrobial therapy: it's not just for mice anymore. Clin Infect Dis. 2007;44(1):79-86.

125. Guiastrennec B, Ramachandran G, Karlsson MO, Kumar AKH, Bhavani PK, Gangadevi NP, et al. Suboptimal Antituberculosis Drug Concentrations and Outcomes in Small and HIV-Coinfected Children in India: Recommendations for Dose Modifications. Clin Pharmacol Ther. 2017.

126. Jayaram R, Shandil RK, Gaonkar S, Kaur P, Suresh BL, Mahesh BN, et al. Isoniazid pharmacokinetics-pharmacodynamics in an aerosol infection model of tuberculosis. Antimicrob Agents Chemother. 2004;48(8):2951-7.

127. Pasipanodya JG, Mcllleron H, Burger A, Wash PA, Smith P, Gumbo T. Serum drug concentrations predictive of pulmonary tuberculosis outcomes. J Infect Dis. 2013;208(9):146473.

128. Pasipanodya JG, Nuermberger E, Romero K, Hanna D, Gumbo T. Systematic Analysis of Hollow Fiber Model of Tuberculosis Experiments. Clin Infect Dis. 2015;61 Suppl 1:S10-7.

129. Pitt JJ. Principles and applications of liquid chromatography-mass spectrometry in clinical biochemistry. Clin Biochem Rev. 2009;30(1):19-34.

130. Kang JS, Lee MH. Overview of therapeutic drug monitoring. Korean J Intern Med. 2009;24(1):110.

131. Eliasson E, Lindh JD, Malmstrom RE, Beck O, Dahl ML. Therapeutic drug monitoring for tomorrow. Eur J Clin Pharmacol. 2013;69 Suppl 1:25-32.

132. Devaleenal Daniel B, Ramachandran G, Swaminathan S. The challenges of pharmacokinetic variability of first-line anti-TB drugs. Expert Rev Clin Pharmacol. 2017;10(1):47-58.

133. Mcllleron $\mathrm{H}$, Chirehwa MT. Current research toward optimizing dosing of first-line antituberculosis treatment. Expert Rev Anti Infect Ther. 2019;17(1):27-38.

134. Holdiness MR. Clinical pharmacokinetics of the antituberculosis drugs. Clin Pharmacokinet. 1984;9(6):511-44.

135. Wilby KJ, Ensom MH, Marra F. Review of evidence for measuring drug concentrations of firstline antitubercular agents in adults. Clin Pharmacokinet. 2014;53(10):873-90.

136. Prahl JB, Johansen IS, Cohen AS, Frimodt-Moller N, Andersen AB. Clinical significance of $2 \mathrm{~h}$ plasma concentrations of first-line anti-tuberculosis drugs: a prospective observational study. J Antimicrob Chemother. 2014;69(10):2841-7.

137. Narita M, Hisada M, Thimmappa B, Stambaugh J, Ibrahim E, Hollender E, et al. Tuberculosis recurrence: multivariate analysis of serum levels of tuberculosis drugs, human immunodeficiency virus status, and other risk factors. Clin Infect Dis. 2001;32(3):515-7.

138. Mota L, Al-Efraij K, Campbell JR, Cook VJ, Marra F, Johnston J. Therapeutic drug monitoring in anti-tuberculosis treatment: a systematic review and meta-analysis. Int J Tuberc Lung Dis. 2016;20(6):819-26.

139. Peloquin CA, Dorman SE, Vernon A, Battista Migliori G, Nahid P. Reply to Alffenaar et al. Clin Infect Dis. 2017;64(1):105-6.

140. van der Burgt EP, Sturkenboom MG, Bolhuis MS, Akkerman OW, Kosterink JG, de Lange WC, et al. End TB with precision treatment! Eur Respir J. 2016;47(2):680-2.

141. Alffenaar JC, Tiberi S, Verbeeck RK, Heysell SK, Grobusch MP. Therapeutic Drug Monitoring in Tuberculosis: Practical Application for Physicians. Clin Infect Dis. 2017;64(1):104-5. 
142. Peloquin C. The Role of Therapeutic Drug Monitoring in Mycobacterial Infections. Microbiol Spectr. 2017;5(1).

143. Dian S, Yunivita V, Ganiem AR, Pramaesya T, Chaidir L, Wahyudi K, et al. Double-Blind, Randomized, Placebo-Controlled Phase II Dose-Finding Study To Evaluate High-Dose Rifampin for Tuberculous Meningitis. Antimicrob Agents Chemother. 2018;62(12).

144. Velasquez GE, Brooks MB, Coit JM, Pertinez H, Vargas Vasquez D, Sanchez Garavito E, et al. ERS Special Article. Efficacy and Safety of High-Dose Rifampin in Pulmonary Tuberculosis. A Randomized Controlled Trial. Am J Respir Crit Care Med. 2018;198(5):657-66.

145. Sekaggya-Wiltshire C, von Braun A, Lamorde M, Ledergerber B, Buzibye A, Henning L, et al. Delayed Sputum Culture Conversion in Tuberculosis-Human Immunodeficiency VirusCoinfected Patients With Low Isoniazid and Rifampicin Concentrations. Clin Infect Dis. 2018;67(5):708-16.

146. Aarnoutse RE, Kibiki GS, Reither K, Semvua HH, Haraka F, Mtabho CM, et al. Pharmacokinetics, Tolerability, and Bacteriological Response of Rifampin Administered at 600, 900, and 1,200 Milligrams Daily in Patients with Pulmonary Tuberculosis. Antimicrob Agents Chemother. 2017;61(11).

147. Alkabab Y, Keller S, Dodge D, Houpt E, Staley D, Heysell S. Early interventions for diabetes related tuberculosis associate with hastened sputum microbiological clearance in Virginia, USA. BMC Infectious Diseases. 2017;17:125.

148. Boeree MJ, Heinrich N, Aarnoutse R, Diacon AH, Dawson R, Rehal S, et al. High-dose rifampicin, moxifloxacin, and SQ109 for treating tuberculosis: a multi-arm, multi-stage randomised controlled trial. Lancet Infect Dis. 2017;17(1):39-49.

149. Rockwood N, Pasipanodya JG, Denti P, Sirgel F, Lesosky M, Gumbo T, et al. ConcentrationDependent Antagonism and Culture Conversion in Pulmonary Tuberculosis. Clin Infect Dis. 2017;64(10):1350-9.

150. Heemskerk AD, Bang ND, Mai NT, Chau TT, Phu NH, Loc PP, et al. Intensified Antituberculosis Therapy in Adults with Tuberculous Meningitis. N Engl J Med. 2016;374(2):124-34.

151. Maze MJ, Paynter J, Chiu W, Hu R, Nisbet M, Lewis C. Therapeutic drug monitoring of isoniazid and rifampicin during anti-tuberculosis treatment in Auckland, New Zealand. Int J Tuberc Lung Dis. 2016;20(7):955-60.

152. Boeree MJ, Diacon AH, Dawson R, Narunsky K, du Bois J, Venter A, et al. A dose-ranging trial to optimize the dose of rifampin in the treatment of tuberculosis. Am J Respir Crit Care Med. 2015;191(9):1058-65.

153. Chigutsa E, Pasipanodya JG, Visser ME, van Helden PD, Smith PJ, Sirgel FA, et al. Impact of nonlinear interactions of pharmacokinetics and MICs on sputum bacillary kill rates as a marker of sterilizing effect in tuberculosis. Antimicrob Agents Chemother. 2015;59(1):38-45.

154. Mah A, Kharrat H, Ahmed R, Gao Z, Der E, Hansen E, et al. Serum drug concentrations of INH and RMP predict 2-month sputum culture results in tuberculosis patients. Int J Tuberc Lung Dis. 2015;19(2):210-5.

155. Meloni M, Corti N, Muller D, Henning L, Gutteck U, von Braun A, et al. Cure of tuberculosis despite serum concentrations of antituberculosis drugs below published reference ranges. Swiss Med Wkly. 2015;145:w14223.

156. Park JS, Lee JY, Lee YJ, Kim SJ, Cho YJ, Yoon HI, et al. Serum Levels of Antituberculosis Drugs and Their Effect on Tuberculosis Treatment Outcome. Antimicrob Agents Chemother. 2016;60(1):92-8. 
157. Burhan E, Ruesen C, Ruslami R, Ginanjar A, Mangunnegoro H, Ascobat P, et al. Isoniazid, rifampin, and pyrazinamide plasma concentrations in relation to treatment response in Indonesian pulmonary tuberculosis patients. Antimicrob Agents Chemother. 2013;57(8):36149.

158. Van Tongeren L, Nolan S, Cook VJ, FitzGerald JM, Johnston JC. Therapeutic drug monitoring in the treatment of tuberculosis: a retrospective analysis. Int J Tuberc Lung Dis. 2013;17(2):221-4.

159. Ruslami R, Ganiem AR, Dian S, Apriani L, Achmad TH, van der Ven AJ, et al. Intensified regimen containing rifampicin and moxifloxacin for tuberculous meningitis: an open-label, randomised controlled phase 2 trial. Lancet Infect Dis. 2013;13(1):27-35.

160. Requena-Méndez A, Davies G, Ardrey A, Jave O, López-Romero SL, Ward SA, et al. Pharmacokinetics of Rifampin in Peruvian Tuberculosis Patients with and without Comorbid Diabetes or HIV. Antimicrobial Agents and Chemotherapy. 2012;56(5):2357-63.

161. Chideya S, Winston CA, Peloquin CA, Bradford WZ, Hopewell PC, Wells CD, et al. Isoniazid, rifampin, ethambutol, and pyrazinamide pharmacokinetics and treatment outcomes among a predominantly HIV-infected cohort of adults with tuberculosis from Botswana. Clin Infect Dis. 2009;48(12):1685-94.

162. Chang KC, Leung CC, Yew WW, Kam KM, Yip CW, Ma CH, et al. Peak plasma rifampicin level in tuberculosis patients with slow culture conversion. Eur J Clin Microbiol Infect Dis. 2008;27(6):467-72.

163. Ruslami R, Nijland HMJ, Alisjahbana B, Parwati I, van Crevel R, Aarnoutse RE. Pharmacokinetics and Tolerability of a Higher Rifampin Dose versus the Standard Dose in Pulmonary Tuberculosis Patients. Antimicrobial Agents and Chemotherapy. 2007;51(7):2546-51.

164. van Crevel R, Alisjahbana B, de Lange WC, Borst F, Danusantoso H, van der Meer JW, et al. Low plasma concentrations of rifampicin in tuberculosis patients in Indonesia. Int J Tuberc Lung Dis. 2002;6(6):497-502.

165. Gumbo T, Louie A, Deziel MR, Liu W, Parsons LM, Salfinger M, et al. Concentration-dependent Mycobacterium tuberculosis killing and prevention of resistance by rifampin. Antimicrob Agents Chemother. 2007;51(11):3781-8.

166. Goutelle S, Bourguignon L, Maire PH, Van Guilder M, Conte JE, Jr., Jelliffe RW. Population modeling and Monte Carlo simulation study of the pharmacokinetics and antituberculosis pharmacodynamics of rifampin in lungs. Antimicrob Agents Chemother. 2009;53(7):2974-81.

167. Prahl JB. Pharamcokinetics and pharmacodynamics of anti-tuberculosis drugs in patients with tuberculosis: Aarhus University; 2014.

168. Gumbo T, Louie A, Liu W, Brown D, Ambrose PG, Bhavnani SM, et al. Isoniazid bactericidal activity and resistance emergence: integrating pharmacodynamics and pharmacogenomics to predict efficacy in different ethnic populations. Antimicrob Agents Chemother. 2007;51(7):2329-36.

169. Gumbo T, Louie A, Deziel MR, Parsons LM, Salfinger M, Drusano GL. Selection of a moxifloxacin dose that suppresses drug resistance in Mycobacterium tuberculosis, by use of an in vitro pharmacodynamic infection model and mathematical modeling. J Infect Dis. 2004;190(9):1642-51.

170. Deshpande D, Pasipanodya JG, Srivastava S, Bendet P, Koeuth T, Bhavnani SM, et al. Gatifloxacin Pharmacokinetics/Pharmacodynamics-based Optimal Dosing for Pulmonary and Meningeal Multidrug-resistant Tuberculosis. Clin Infect Dis. 2018;67(suppl_3):S274-s83. 
171. Ellington MJ, Ekelund O, Aarestrup FM, Canton R, Doumith M, Giske C, et al. The role of whole genome sequencing in antimicrobial susceptibility testing of bacteria: report from the EUCAST Subcommittee. Clin Microbiol Infect. 2017;23(1):2-22.

172. Schon T, Matuschek E, Mohamed S, Utukuri M, Heysell S, Alffenaar JW, et al. Standards for MIC testing that apply to the majority of bacterial pathogens should also be enforced for Mycobacterium tuberculosis complex. Clin Microbiol Infect. 2019.

173. Sturegard E, Angeby KA, Werngren J, Jureen P, Kronvall G, Giske CG, et al. Little difference between minimum inhibitory concentrations of Mycobacterium tuberculosis wild-type organisms determined with BACTEC MGIT 960 and Middlebrook 7H10. Clin Microbiol Infect. 2015;21(2):148.e5-7.

174. Rastogi N, Goh KS, David HL. Drug susceptibility testing in tuberculosis: a comparison of the proportion methods using Lowenstein-Jensen, Middlebrook $7 \mathrm{H} 10$ and $7 \mathrm{H} 11$ agar media and a radiometric method. Res Microbiol. 1989;140(6):405-17.

175. Zhang Y, Permar S, Sun Z. Conditions that may affect the results of susceptibility testing of Mycobacterium tuberculosis to pyrazinamide. J Med Microbiol. 2002;51(1):42-9.

176. Salfinger M, Heifets LB. Determination of pyrazinamide MICs for Mycobacterium tuberculosis at different pHs by the radiometric method. Antimicrob Agents Chemother. 1988;32(7):10024.

177. Ajileye A, Alvarez N, Merker M, Walker TM, Akter S, Brown K, et al. Some Synonymous and Nonsynonymous gyrA Mutations in Mycobacterium tuberculosis Lead to Systematic FalsePositive Fluoroquinolone Resistance Results with the Hain GenoType MTBDRsl Assays. Antimicrob Agents Chemother. 2017;61(4).

178. Kaswa MK, Aloni M, Nkuku L, Bakoko B, Lebeke R, Nzita A, et al. Pseudo-outbreak of preextensively drug-resistant (Pre-XDR) tuberculosis in Kinshasa: collateral damage caused by false detection of fluoroquinolone resistance by GenoType MTBDRsI. J Clin Microbiol. 2014;52(8):2876-80.

179. Sirgel FA, Warren RM, Streicher EM, Victor TC, van Helden PD, Bottger EC. gyrA mutations and phenotypic susceptibility levels to ofloxacin and moxifloxacin in clinical isolates of Mycobacterium tuberculosis. J Antimicrob Chemother. 2012;67(5):1088-93.

180. Rigouts L, Coeck N, Gumusboga M, de Rijk WB, Aung KJ, Hossain MA, et al. Specific gyrA gene mutations predict poor treatment outcome in MDR-TB. J Antimicrob Chemother. 2016;71(2):314-23.

181. Maruri F, Sterling TR, Kaiga AW, Blackman A, van der Heijden YF, Mayer C, et al. A systematic review of gyrase mutations associated with fluoroquinolone-resistant Mycobacterium tuberculosis and a proposed gyrase numbering system. J Antimicrob Chemother. 2012;67(4):819-31.

182. Kruuner A, Yates MD, Drobniewski FA. Evaluation of MGIT 960-based antimicrobial testing and determination of critical concentrations of first- and second-line antimicrobial drugs with drugresistant clinical strains of Mycobacterium tuberculosis. J Clin Microbiol. 2006;44(3):811-8.

183. Angeby K, Giske CG, Jureen P, Schon T, Pasipanodya JG, Gumbo T. Wild-type MIC distributions must be considered to set clinically meaningful susceptibility testing breakpoints for all bacterial pathogens, including Mycobacterium tuberculosis. Antimicrob Agents Chemother. 2011;55(9):4492-3; author reply 3.

184. Van Deun A, Aung KJ, Bola V, Lebeke R, Hossain MA, de Rijk WB, et al. Rifampin drug resistance tests for tuberculosis: challenging the gold standard. J Clin Microbiol. 2013;51(8):2633-40. 
185. Rigouts L, Gumusboga M, de Rijk WB, Nduwamahoro E, Uwizeye C, de Jong B, et al. Rifampin resistance missed in automated liquid culture system for Mycobacterium tuberculosis isolates with specific rpoB mutations. J Clin Microbiol. 2013;51(8):2641-5.

186. Bottger EC. The ins and outs of Mycobacterium tuberculosis drug susceptibility testing. Clin Microbiol Infect. 2011;17(8):1128-34.

187. Angeby KA, Jureen P, Giske CG, Chryssanthou E, Sturegard E, Nordvall M, et al. Wild-type MIC distributions of four fluoroquinolones active against Mycobacterium tuberculosis in relation to current critical concentrations and available pharmacokinetic and pharmacodynamic data. J Antimicrob Chemother. 2010;65(5):946-52.

188. Peloquin CA, Hadad DJ, Molino LP, Palaci M, Boom WH, Dietze R, et al. Population pharmacokinetics of levofloxacin, gatifloxacin, and moxifloxacin in adults with pulmonary tuberculosis. Antimicrob Agents Chemother. 2008;52(3):852-7.

189. Van't Boveneind-Vrubleuskaya N, Seuruk T, van Hateren K, van der Laan T, Kosterink JGW, van der Werf TS, et al. Pharmacokinetics of Levofloxacin in Multidrug- and Extensively DrugResistant Tuberculosis Patients. Antimicrob Agents Chemother. 2017;61(8).

190. Ghimire S, Van't Boveneind-Vrubleuskaya N, Akkerman OW, de Lange WC, van Soolingen D, Kosterink JG, et al. Pharmacokinetic/pharmacodynamic-based optimization of levofloxacin administration in the treatment of MDR-TB. J Antimicrob Chemother. 2016;71(10):2691-703.

191. Alsultan A, An G, Peloquin CA. Limited sampling strategy and target attainment analysis for levofloxacin in patients with tuberculosis. Antimicrob Agents Chemother. 2015;59(7):3800-7.

192. Gorelik E, Masarwa R, Perlman A, Rotshild V, Abbasi M, Muszkat M, et al. Fluoroquinolones and Cardiovascular Risk: A Systematic Review, Meta-analysis and Network Meta-analysis. Drug Saf. 2018.

193. Ghimire S, Maharjan B, Jongedijk EM, Kosterink JGW, Ghimire GR, Touw DJ, et al. Levofloxacin pharmacokinetics and pharmacodynamics and outcome in MDR-TB patients. Eur Respir J. 2019.

194. Bouton TC, Phillips PPJ, Mitnick CD, Peloquin CA, Eisenach K, Patientia RF, et al. An optimized background regimen design to evaluate the contribution of levofloxacin to multidrug-resistant tuberculosis treatment regimens: study protocol for a randomized controlled trial. Trials. 2017;18(1):563.

195. Cox H, Mizrahi V. The Coming of Age of Drug-Susceptibility Testing for Tuberculosis. N Engl J Med. 2018;379(15):1474-5.

196. Heyckendorf J, Andres S, Koser CU, Olaru ID, Schon T, Sturegard E, et al. What Is Resistance? Impact of Phenotypic versus Molecular Drug Resistance Testing on Therapy for Multi- and Extensively Drug-Resistant Tuberculosis. Antimicrob Agents Chemother. 2018;62(2).

197. Koser CU, Heyckendorf J, Andres S, Olaru ID, Schon T, Sturegard E, et al. Reply to Dookie et al., "Whole-Genome Sequencing To Guide the Selection of Treatment for Drug-Resistant Tuberculosis". Antimicrob Agents Chemother. 2018;62(8).

198. Peloquin CA, Velasquez GE, Lecca L, Calderon RI, Coit J, Milstein M, et al. Pharmacokinetic Evidence from the HIRIF Trial To Support Increased Doses of Rifampin for Tuberculosis. Antimicrob Agents Chemother. 2017;61(8).

199. Sturkenboom MG, Bolhuis MS, Akkerman OW, de Lange WC, van der Werf TS, Alffenaar JC. Therapeutic drug monitoring of first-line anti-tuberculosis drugs comprises more than $\mathrm{C} 2 \mathrm{~h}$ measurements. Int J Tuberc Lung Dis. 2016;20(12):1695-6. 
200. Magis-Escurra C, Later-Nijland HM, Alffenaar JW, Broeders J, Burger DM, van Crevel R, et al. Population pharmacokinetics and limited sampling strategy for first-line tuberculosis drugs and moxifloxacin. Int J Antimicrob Agents. 2014;44(3):229-34.

201. Ellard GA. Variations between individuals and populations in the acetylation of isoniazid and its significance for the treatment of pulmonary tuberculosis. Clin Pharmacol Ther. 1976;19(5 Pt 2):610-25.

202. Smith CA, Wadelius M, Gough AC, Harrison DJ, Wolf CR, Rane A. A simplified assay for the arylamine $\mathrm{N}$-acetyltransferase 2 polymorphism validated by phenotyping with isoniazid. J Med Genet. 1997;34(9):758-60.

203. Azuma J, Ohno M, Kubota R, Yokota S, Nagai T, Tsuyuguchi K, et al. NAT2 genotype guided regimen reduces isoniazid-induced liver injury and early treatment failure in the 6-month fourdrug standard treatment of tuberculosis: a randomized controlled trial for pharmacogeneticsbased therapy. Eur J Clin Pharmacol. 2013;69(5):1091-101.

204. Peloquin CA, Namdar R, Singleton MD, Nix DE. Pharmacokinetics of rifampin under fasting conditions, with food, and with antacids. Chest. 1999;115(1):12-8.

205. Chen J, Raymond K. Roles of rifampicin in drug-drug interactions: underlying molecular mechanisms involving the nuclear pregnane $X$ receptor. Ann Clin Microbiol Antimicrob. 2006;5:3.

206. Acocella G. Pharmacokinetics and metabolism of rifampin in humans. Rev Infect Dis. 1983;5 Suppl 3:S428-32.

207. Acocella G. Clinical pharmacokinetics of rifampicin. Clin Pharmacokinet. 1978;3(2):108-27.

208. Loos U, Musch E, Jensen JC, Mikus G, Schwabe HK, Eichelbaum M. Pharmacokinetics of oral and intravenous rifampicin during chronic administration. Klin Wochenschr. 1985;63(23):120511.

209. Svensson RJ, Aarnoutse RE, Diacon AH, Dawson R, Gillespie SH, Boeree MJ, et al. A Population Pharmacokinetic Model Incorporating Saturable Pharmacokinetics and Autoinduction for High Rifampicin Doses. Clin Pharmacol Ther. 2018;103(4):674-83.

210. Swaminathan S, Pasipanodya JG, Ramachandran G, Hemanth Kumar AK, Srivastava S, Deshpande D, et al. Drug Concentration Thresholds Predictive of Therapy Failure and Death in Children With Tuberculosis: Bread Crumb Trails in Random Forests. Clin Infect Dis. 2016;63(suppl 3):S63-s74.

211. Te Brake L, Dian S, Ganiem AR, Ruesen C, Burger D, Donders R, et al. Pharmacokinetic/pharmacodynamic analysis of an intensified regimen containing rifampicin and moxifloxacin for tuberculous meningitis. Int J Antimicrob Agents. 2015;45(5):496-503.

212. Donald PR. Cerebrospinal fluid concentrations of antituberculosis agents in adults and children. Tuberculosis (Edinb). 2010;90(5):279-92.

213. Zuercher K BM, Fenner L, Borrell S, Keller PM, Gnokoro J, Marcy O, Yotebieng M, Diero L, Carter EJ, Rockwood N, Wilkinson RJ, Cox H, Ezati N, Abimiku AG, Collantes J, Avihingsanon A, Kawkitinarong K, Reinhard M, Hömke R, Huebner R, Gagneux S, Böttger EC, Egger M, on behalf of the International Epidemiology Databases to Evaluate AIDS (leDEA). Drug susceptibility testing and mortality in patients treated for tuberculosis in high-burden countries bioRxiv. 2018.

214. Davies Forsman L, Bruchfeld J, Alffenaar JC. Therapeutic drug monitoring to prevent acquired drug resistance of fluoroquinolones in the treatment of tuberculosis. Eur Respir J. 2017;49(4). 
215. Mouton JW, Muller AE, Canton R, Giske CG, Kahlmeter G, Turnidge J. MIC-based dose adjustment: facts and fables. J Antimicrob Chemother. 2017.

216. Gumbo T, Pasipanodya JG, Wash P, Burger A, Mcllleron H. Redefining multidrug-resistant tuberculosis based on clinical response to combination therapy. Antimicrob Agents Chemother. 2014;58(10):6111-5.

217. Colangeli R, Jedrey H, Kim S, Connell R, Ma S, Chippada Venkata UD, et al. Bacterial Factors That Predict Relapse after Tuberculosis Therapy. N Engl J Med. 2018;379(9):823-33.

218. Budha NR, Lee RE, Meibohm B. Biopharmaceutics, pharmacokinetics and pharmacodynamics of antituberculosis drugs. Curr Med Chem. 2008;15(8):809-25.

219. Srivastava S, Pasipanodya JG, Meek C, Leff R, Gumbo T. Multidrug-resistant tuberculosis not due to noncompliance but to between-patient pharmacokinetic variability. J Infect Dis. 2011;204(12):1951-9.

220. Mpagama SG, Ndusilo N, Stroup S, Kumburu H, Peloquin CA, Gratz J, et al. Plasma drug activity in patients on treatment for multidrug-resistant tuberculosis. Antimicrob Agents Chemother. 2014;58(2):782-8.

221. Lee SH, Seo KA, Lee YM, Lee HK, Kim JH, Shin C, et al. Low Serum Concentrations of Moxifloxacin, Prothionamide, and Cycloserine on Sputum Conversion in Multi-Drug Resistant TB. Yonsei Med J. 2015;56(4):961-7.

222. Srivastava S. Minimum inhibitory concentration, pharmacokinetics/pharmacodynamics and therapeutic drug monitoring: An integrated approach for multidrug-resistant tuberculosis. Lung India. 2015;32(4):402-3.

223. Heysell SK, Mtabho C, Mpagama S, Mwaigwisya S, Pholwat S, Ndusilo N, et al. Plasma drug activity assay for treatment optimization in tuberculosis patients. Antimicrob Agents Chemother. 2011;55(12):5819-25.

224. CRYPTIC. Comprehensive Resistance Prediction for Tuberculosis: an International Consortium. [updated October 24th, 2018]

225. The EUCAST Subcommittee on Antimycobacterial Susceptibility Testing. Workshop on recommendations for pharmaceutical companies regarding data required for new antituberculous drugs. 2014. Available from: http://www.eucast.org/fileadmin/src/media/PDFs/EUCAST files/Mycobacteria/Documents/M ycobacterial workshop Basel 11-12Nov2014 report.pdf.

226. Srivastava S, Deshpande D, Magombedze G, Gumbo T. Efficacy Versus Hepatotoxicity of Highdose Rifampin, Pyrazinamide, and Moxifloxacin to Shorten Tuberculosis Therapy Duration: There Is Still Fight in the Old Warriors Yet! Clin Infect Dis. 2018;67(suppl_3):S359-s64.

227. Burki T. PanACEA: a new approach to tuberculosis research. Lancet Infect Dis. 2012;12(3):1845.

228. Grace AG, Mittal A, Jain S, Tripathy JP, Satyanarayana S, Tharyan P, et al. Shortened treatment regimens versus the standard regimen for drug-sensitive pulmonary tuberculosis. Cochrane Database of Systematic Reviews 2018, Issue 1. Art. No.: CD012918. DOI: 10.1002/14651858.CD012918. 2018.

229. Goletti D, Lindestam Arlehamn CS, Scriba TJ, Anthony R, Cirillo DM, Alonzi T, et al. Can we predict tuberculosis cure? What tools are available? Eur Respir J. 2018;52(5).

230. O'Garra A, Redford PS, McNab FW, Bloom Cl, Wilkinson RJ, Berry MP. The immune response in tuberculosis. Annu Rev Immunol. 2013;31:475-527. 
231. Bruchfeld J, Correia-Neves M, Kallenius G. Tuberculosis and HIV Coinfection. Cold Spring Harb Perspect Med. 2015;5(7):a017871.

232. Mahomed S, Naidoo K, Dookie N, Padayatchi N. Whole genome sequencing for the management of drug-resistant TB in low income high TB burden settings: Challenges and implications. Tuberculosis (Edinb). 2017;107:137-43.

233. Vu DH, Alffenaar JW, Edelbroek PM, Brouwers JR, Uges DR. Dried blood spots: a new tool for tuberculosis treatment optimization. Curr Pharm Des. 2011;17(27):2931-9. 


\section{Papers}

The papers associated with this thesis have been removed for copyright reasons. For more details about these see:

http://urn.kb.se/resolve?urn=urn:nbn:se:liu:diva-156494 


\section{FACULTY OF MEDICINE AND HEALTH SCIENCES}

Linköping University Medical Dissertation No. 1662, 2019 Department of Infectious Diseases

Department of Clinical and Experimental Medicine

Linköping University

SE-581 83 Linköping, Sweden

www.liu.se 